\title{
Querying Log Data with Metric Temporal Logic
}

\author{
Sebastian Brandt \\ Siemens $C T$ \\ München, Germany
}

Elem Güzel Kalaycı

KRDB Research Centre

Faculty of Computer Science

Free University of Bozen-Bolzano, Italy

Vladislav Ryzhikov

Department of Computer Science and Information Systems

Birkbeck, University of London, UK

Guohui Xiao

KRDB Research Centre

Faculty of Computer Science

Free University of Bozen-Bolzano, Italy

Michael Zakharyaschev

Department of Computer Science and Information Systems

Birkbeck, University of London, UK
SEBASTIAN-PHILIPP.BRANDT@SIEMENS.COM

KALAYCI@INF.UNIBZ.IT

VLAD@DCS.BBK.AC.UK

XIAO@INF.UNIBZ.IT

MICHAEL@DCS.BBK.AC.UK

\begin{abstract}
We propose a novel framework for ontology-based access to temporal log data using a datalog extension datalogMTL of the Horn fragment of the metric temporal logic MTL. We show that datalogMTL is EXPSPACE-complete even with punctual intervals, in which case full MTL is known to be undecidable. We also prove that nonrecursive datalogMTL is PSPACE-complete for combined complexity and in $\mathrm{AC}^{0}$ for data complexity. We demonstrate by two real-world use cases that nonrecursive datalogMTL programs can express complex temporal concepts from typical user queries and thereby facilitate access to temporal log data. Our experiments with Siemens turbine data and MesoWest weather data show that datalogMTL ontology-mediated queries are efficient and scale on large datasets.
\end{abstract}

\section{Introduction}

In this paper, we present a new ontology-based framework for querying temporal log data. We begin by outlining this framework in the context of data gathering and analysis at Siemens, a leading manufacturer and supplier of systems for power generation, power transmission, medical diagnosis, and industry automation.

\subsection{Data Gathering at Siemens}

For the Siemens equipment, analytics services are usually delivered by remote diagnostic centres that store data from the relevant industrial sites or individual equipment around the globe. The analytics provided at these centres falls into three categories: descriptive, predictive, and prescriptive. Descriptive analytics describes or quantifies in detail what has happened after an event. Predictive 
analytics aims to anticipate events before they occur and provide a window of opportunity for countermeasures. Prescriptive analytics aims to automate the process of suggesting underlying reasons for the predicted events and carrying out appropriate countermeasures. All these types of analytics heavily rely on the ability to recognise interesting events using sensor measurements or other machine data such as the power output of a gas turbine, its maximum rotor speed, average exhaust temperature, etc. For example, a service engineer at a Siemens remote diagnostic centre could be interested in active power trips of the turbine, that is, events when

(ActivePowerTrip) the active power was above $1.5 \mathrm{MW}$ for a period of at least 10 seconds, maximum 3 seconds after which there was a period of at least one minute where the active power was below $0.15 \mathrm{MW}$.

Under the standard workflow, when facing the task of finding the active power trips of the turbine, the engineer would call an IT expert who would then produce a specific script (in a proprietary signal processing language developed by Siemens) such as

$$
\begin{aligned}
& \text { message("active power trip") }= \\
& \$ t 1: \text { eval }(>, \text { \#activePower, } 1.5): \\
& \quad \text { for }(>=10 s) \\
& \quad \& \& \\
& \quad \text { eval }(<, \# \text { activePower, } 0.15): \\
& \quad \operatorname{start~}(\text { after }[0 s, 3 s] \$ t 1: \text { end }): \\
& \quad \text { for }(>=1 m) ;
\end{aligned}
$$

for the turbine aggregated data stored in a table TB_Sensor, which looks as follows:

\begin{tabular}{|c|c|c|c|c|c|}
\hline turbineId & dateTime & activePower & rotorSpeed & mainFlame & $\ldots$ \\
\hline & & $\ldots$ & & & \\
tb0 & $2015-04-0412: 20: 48$ & 2 & 1550 & 0 & \\
tb0 & $2015-04-0412: 20: 49$ & 1.8 & 1400 & null & \\
tb0 & $2015-04-0412: 20: 52$ & 1.7 & 1350 & 1 & \\
& & $\ldots$ & & & \\
\hline
\end{tabular}

The result of running the script is a log with records such as

"2015-04-04 12:22:17 active power trip tb0"

where information about all the events is accumulated.

When facing the same task but for a different turbine, the engineer may have to call the IT expert once again because different models of turbines and sensors may have different $\log / \mathrm{database}$ formats. Moreover, the storage platform for the sensor data often changes (thus, currently Siemens are pondering over migrating certain data to a cloud-based storage). Maintaining a set of scripts, one for each data source, does not provide an efficient solution since a query such as 'find all the turbines that had an active power trip in May 2017' would require an intermediate database with integrated data of active power trips. Another difficulty is that the definitions of events the engineer is interested in can also change. Some changes are minor, say the pressure threshold or the number of seconds in the active power trip definition, but some could be more substantial, such as 'find the 
active power trips that were followed by a high pressure within 3 minutes that lasted for 30 seconds'. This modification would require rewriting the script above into a much longer one rather than using it as a module in the new definition.

The permanent involvement of an IT expert familiar with database technology incurs high costs for Siemens, and data gathering accounts for a major part of the time the service engineers spend at Siemens remote diagnostic centres, most of which due to the indirect access to data.

\subsection{Ontology-Based Data Access}

Ontology-based data access (OBDA for short) offers a different workflow that excludes the IT middleman from data gathering (Poggi, Lembo, Calvanese, De Giacomo, Lenzerini, \& Rosati, 2008); consult also the recent survey by Xiao, Calvanese, Kontchakov, Lembo, Poggi, Rosati, and Zakharyaschev (2018). In a nutshell, the OBDA workflow in the Siemens context looks as follows. Domain experts develop and maintain an ontology that contains terms for the events the engineers may be interested in. IT experts develop and maintain mappings that relate these terms to the database schemas. The engineer can now use familiar terms from the ontology and a graphical tool such as OptiqueVQS (Soylu, Giese, Jiménez-Ruiz, Vega-Gorgojo, \& Horrocks, 2016) to construct and run queries such as ActivePowerTrip (tb0)@x. The task of the OBDA system such as Ontop (Rodriguez-Muro, Kontchakov, \& Zakharyaschev, 2013; Calvanese, Cogrel, Komla-Ebri, Kontchakov, Lanti, Rezk, Rodriguez-Muro, \& Xiao, 2017) will be, using the mappings, to rewrite the engineer's ontology-mediated query into an SQL query over the database and then execute it returning the time intervals $x$ where the turbine with the ID tb0 had active power trips.

Unfortunately, the ontology and query languages designed for OBDA and standardised by the W3C - the OWL 2 QL profile of OWL 2 and SPARQL - are not suitable for the Siemens case because they were not meant to deal with essentially temporal data, concepts and properties. There have been several attempts to develop temporal OBDA.

One approach is to use the same $O W L 2 Q L$ as an ontology language, assuming that ontology axioms hold at all times, and extend the query language with various temporal operators (GutiérrezBasulto \& Klarman, 2012; Baader, Borgwardt, \& Lippmann, 2013; Borgwardt, Lippmann, \& Thost, 2013; Özçep, Möller, Neuenstadt, Zheleznyakov, \& Kharlamov, 2013; Klarman \& Meyer, 2014; Özçep \& Möller, 2014; Kharlamov, Brandt, Jiménez-Ruiz, Kotidis, Lamparter, Mailis, Neuenstadt, Özçep, Pinkel, Svingos, Zheleznyakov, Horrocks, Ioannidis, \& Möller, 2016). Unfortunately, $O W L 2 Q L$ is not able to define the temporal feature of 'active power trip', and so the engineer would have to capture it in a complex temporal query (or call an expert in temporal logic). Another known approach is to allow the temporal operators of the linear-time temporal logic LTL in both queries and ontologies (Artale, Kontchakov, Wolter, \& Zakharyaschev, 2013; Artale, Kontchakov, Kovtunova, Ryzhikov, Wolter, \& Zakharyaschev, 2015; Gutiérrez-Basulto, Jung, \& Kontchakov, 2016a). For more details and further references, consult the recent survey by Artale, Kontchakov, Kovtunova, Ryzhikov, Wolter, and Zakharyaschev (2017) ${ }^{1}$.

However, standard LTL over a discrete timeline such as $(\mathbb{N}, \leq)$ or $(\mathbb{Z}, \leq)$ is not able to adequately represent the temporal data and knowledge in the Siemens use case because measurements are taken and sent asynchronously by multiple sensors at irregular time intervals, which can depend on the turbine model, sensor type, etc. To model measurements and events using discrete time, one

1. Surveys of early developments in temporal deductive databases are given by Baudinet, Chomicki, and Wolper (1993) and Chomicki and Toman (1998). 
could take a sufficiently small time unit (quantum), say 1 second, and encode 'active power was below $0.15 \mathrm{MW}$ for a period of one minute' by an $L T L$-formula of the form $\bigcirc_{P} p \wedge \bigcirc_{P}^{2} p \wedge \cdots \wedge \bigcirc_{P}^{60} p$, where $\bigcirc_{P}$ is the previous-time operator. One problem with this encoding is that it is clearly awkward, not succinct, and only works under the assumption that the active power is measured each and every second. If, for some reason, a measurement is missing as in TB_Sensor, the formula becomes inadequate. This problem can be solved by using the (more succinct) metric temporal logic MTL with operators like $\boxminus_{[1,60]}$ interpreted as 'at every time instant within the previous minute when a measurement was taken'. The satisfiability problem for the description logic $\mathcal{A L C}$ extended with such operators over $(\mathbb{N}, \leq)$ was investigated by Gutiérrez-Basulto, Jung, and Ozaki (2016b). A more fundamental issue with modelling turbine events using discrete time is that it only applies to data complying with the chosen quantum and requires amendments every time the quantum has to be set to a different value because of a new equipment or because asynchronous sensor measurements start to happen more frequently. Thus, a better way of modelling the temporal data and events under consideration is by means of a suitable fragment of MTL interpreted over dense time such as the rationals $(\mathbb{Q}, \leq)$ or reals $(\mathbb{R}, \leq)$. This would allow us to capture, for example, that one event, say a sharp temperature rise, happened just before (maybe a fraction of a quantum), and so possibly caused another event, say an emergency shutdown, which is a typical feature of an asynchronous behaviour of real-time systems where the actual time of event occurrences cannot be predicted at the modelling stage.

\subsection{Metric Temporal Logic}

The metric temporal logic MTL was originally designed for modelling and reasoning about realtime systems (Koymans, 1990; Alur \& Henzinger, 1993). MTL is equipped with two alternative semantics, pointwise and continuous (aka interval-based). In both semantics, the timestamps are taken from a dense timeline $(\mathbb{T}, \leq)$ such as $(\mathbb{Q}, \leq)$ or $(\mathbb{R}, \leq)$. Under the pointwise semantics, an interpretation is a timed word, that is, a finite or infinite sequence of pairs $\left(\Sigma_{i}, t_{i}\right)$, where $\Sigma_{i}$ is a subset of propositional variables that are assumed to hold at $t_{i} \in \mathbb{T}$ and $t_{i}<t_{j}$ for $i<j$. Under the continuous semantics, an interpretation is an assignment of a set of propositional variables to each $t \in \mathbb{T}$. MTL allows formulas such as $\boxplus_{[1.5,3]} \varphi$ (or $\left.\bigoplus_{[1.5,3]} \varphi\right)$ that holds at a moment $t$ if and only if $\varphi$ holds at every (respectively, some) moment in the interval $[t+1.5, t+3]$. However, under the pointwise semantics, $t$ must be a timestamp from the timed word and $\varphi$ must only hold at every (respectively, some) $t_{i}$ with $1.5 \leq t_{i}-t \leq 3$. Thus, $\boxplus_{[1,1]} \perp$ is satisfiable under the pointwise semantics, for example, by a timed word with $t_{i+1}-t_{i}>1$, but not under the continuous semantics.

In the Siemens case, we assume that the real-time system is being continuously monitored, the result of the next measurement of a sensor is only recorded when it exceeds the previous one by some fixed margin, and events such as active power trip can happen between measurements. This makes the continuous semantics a natural choice for temporal modelling. The satisfiability problem for MTL under this semantics turns out to be undecidable (Alur \& Henzinger, 1993) and EXPSPACEcomplete if the punctual operators such as $\bigoplus_{[1,1]}$ are disallowed (Alur, Feder, \& Henzinger, 1996); see also the work by Ouaknine and Worrell $(2005,2008)$. Note that, under the pointwise semantics, MTL is decidable over finite timed words, though not primitive recursive (Ouaknine \& Worrell, 2005). 


\subsection{Our Contribution}

Having analysed two real-world scenarios of querying asynchronous real-time systems (to be discussed in Section 6), we came to a conclusion that a basic ontology language for temporal OBDA should contain datalog rules with MTL operators in their bodies. In this language, for example, the event of active power trip can be defined by the rule

$$
\begin{aligned}
\operatorname{ActivePowerTrip}(v) \leftarrow \operatorname{Turbine}(v) \wedge \Xi_{[0,1 m]} & \operatorname{ActivePowerBelow0.15}(v) \wedge \\
& \otimes_{[60 s, 63 s]} \boxminus_{[0,10 s]} \operatorname{ActivePowerAbove1.5}(v) .
\end{aligned}
$$

The variables of the predicates in such rules range over a (non-temporal) object domain. Thus, the intended domain for $v$ in (1) comprises turbines, their parts, sensors, etc. The underlying (dense) timeline is implicit: we understand (1) as saying that $\operatorname{ActivePowerTrip}(v)$ holds at any given time instant $t$ if the pattern shown in the picture below has occurred before $t$ :

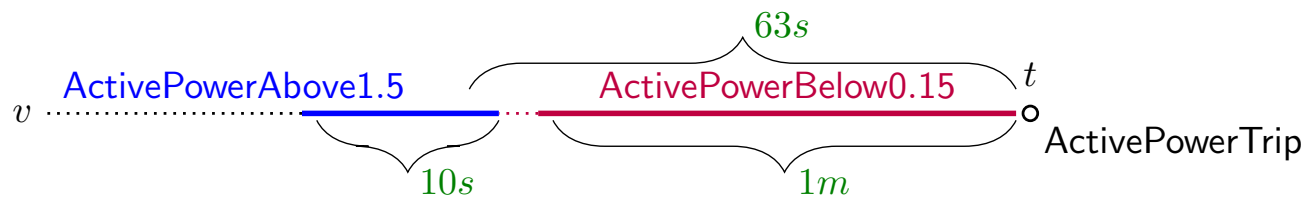

Unlike model-checking liveness properties (that some events eventually happen) in transition systems, our task is to query historical data for events that have already happened and are actually implicitly recorded in the data. As a consequence, we do not need ontology axioms with eventuality operators in the head such as $\oslash_{[0,3 s]} \operatorname{ShutDown}(v) \leftarrow \operatorname{ActivePowerTrip}(v)$ saying that an active power trip must be followed by a shutdown within 3 seconds. OWL 2 QL allows existential quantification in the head of rules such as $\exists u$ hasRotor $(v, u) \leftarrow \operatorname{Turbine}(v)$ stating that every turbine has a rotor. Although axioms of this sort are present in the Siemens turbine configuration ontology (Kharlamov, Mailis, Mehdi, Neuenstadt, Özçep, Roshchin, Solomakhina, Soylu, Svingos, Brandt, Giese, Ioannidis, Lamparter, Möller, Kotidis, \& Waaler, 2017), we opted not to include $\exists$ in the head of rules in our language. On the one hand, we have not found meaningful queries in the use cases for which such axioms would provide more answers. On the other hand, it is known that existential axioms may considerably increase the combined complexity of both atemporal (Gottlob, Kikot, Kontchakov, Podolskii, Schwentick, \& Zakharyaschev, 2014; Bienvenu, Kikot, Kontchakov, Podolskii, \& Zakharyaschev, 2018) and temporal ontology-mediated query answering (Artale et al., 2015). For these reasons, we do not allow existential rules in our ontology language and leave their investigation for future work.

The resulting temporal ontology language can be described as a datalog extension of the Horn fragment of MTL (without diamond operators in the head of rules). We denote this language by datalogMTL and prove in Section 3 that answering ontology-mediated queries of the form $(\Pi, Q(\boldsymbol{v}) @ x)$ is EXPSPACE-complete for combined complexity, where $\Pi$ is a datalogMTL program, $Q(\boldsymbol{v})$ a goal with individual variables $\boldsymbol{v}$, and $x$ a variable over time intervals during which $Q(v)$ holds. On the other hand, we show that hornMTL becomes undecidable if the diamond operators are allowed in the head of rules. We also prove that answering propositional datalogMTL queries is P-hard for data complexity. To compare, recall that answering ontology-mediated queries with propositional (not necessarily Horn) LTL ontologies is $\mathrm{NC}^{1}$-complete for data complexity (Artale et al., 2015). 
From the practical point of view, most interesting are nonrecursive datalogMTL queries. We show in Section 4 that answering such queries is in $\mathrm{AC}^{0}$ for data complexity (assuming that data timestamps and the ranges of the temporal operators in datalogMTL programs are represented as finite binary fractions) and PSPACE-complete for combined complexity (even NP-complete if the arity of predicates is bounded). In this case, we develop a query answering algorithm that can be implemented in standard SQL with window functions. We also present in Section 5 a framework for practical OBDA with nonrecursive datalogMTL queries and temporal log data stored in databases as shown above. Finally, in Section 6, we evaluate our framework on two use cases. We develop a datalogMTL ontology for temporal concepts used in typical queries at Siemens (e.g., NormalStop that takes place if events ActivePowerOff, MainFlameOff, CoastDown6600to1500, and CoastDown1500to200 happen in a certain temporal pattern). We also create a weather ontology defining standard meteorological concepts such as Hurricane (HurricaneForceWind, wind with the speed above $118 \mathrm{~km} / \mathrm{h}$, lasting at least 1 hour). Using Siemens sensor databases and MesoWest historical records of the weather stations across the US, we experimentally demonstrate that our algorithm is efficient in practice and scales on large datasets of up to 8.3GB. We used two systems, PostgreSQL and Apache Spark, to evaluate our SQL programs. To our surprise, Apache Spark achieved tenfold better performance on the weather data than PostgreSQL. This effect can be attributed to the capacity of Spark to parallelise query execution as well as to the natural 'modularity' of weather data by location.

An extended abstract of this paper was presented at AAAI-17 (Brandt, Kalayc1, Kontchakov, Ryzhikov, Xiao, \& Zakharyaschev, 2017).

\section{DatalogMTL}

In the standard metric temporal logic MTL (Alur et al., 1996), the temporal domain is the real numbers $\mathbb{R}$, while the intervals $\varrho$ in the constrained temporal operators such as $\bigoplus_{\varrho}$ (sometime in the future within the interval $\varrho$ from now) have natural numbers or $\infty$ as their endpoints. In the context of the applications of MTL we deal with in this paper, it is more natural to assume that the endpoints of $\varrho$ are non-negative dyadic rational numbers - finite binary fractions ${ }^{2}$ such as 101.011 - or $\infty$. We denote the set of dyadic rationals by $\mathbb{Q}_{2}$ and remind the reader that $\mathbb{Q}_{2}$ is dense in $\mathbb{R}$ and, by Cantor's theorem, $\left(\mathbb{Q}_{2},<\right)$ is isomorphic to $(\mathbb{Q},<)$. By an interval, $\iota$, we mean any nonempty subset of $\mathbb{Q}_{2}$ of the form $\left[t_{1}, t_{2}\right],\left[t_{1}, t_{2}\right),\left(t_{1}, t_{2}\right]$ or $\left(t_{1}, t_{2}\right)$, where $t_{1}, t_{2} \in \mathbb{Q}_{2} \cup\{-\infty, \infty\}$ and $t_{1} \leq t_{2}$. We identify $(t, \infty]$ with $(t, \infty),[-\infty, t]$ with $(-\infty, t]$, etc. A range, $\varrho$, is an interval with non-negative endpoints. The temporal operators of $M T L$ take the form $\boxplus_{\varrho}, \ominus_{\varrho}$ and $\mathcal{U}_{\varrho}$, which refer to the future, and $\boxminus_{\varrho}, \vartheta_{\varrho}$ and $\mathcal{S}_{\varrho}$, which refer to the past. The end-points of intervals and ranges are assumed to be represented in binary.

An individual term, $\tau$, is an individual variable, $v$, or a constant, $c$. As usual, we assume that there is a countably-infinite list of predicate symbols, $P$, with assigned arities. A datalogMTL program, $\Pi$, is a finite set of rules of the form

$$
A^{+} \leftarrow A_{1} \wedge \cdots \wedge A_{k} \quad \text { or } \quad \perp \leftarrow A_{1} \wedge \cdots \wedge A_{k}
$$

where $k \geq 1$, each $A_{i}(1 \leq i \leq k)$ is either an inequality $\left(\tau \neq \tau^{\prime}\right)$ or defined by the grammar

$$
A::=P\left(\tau_{1}, \ldots, \tau_{m}\right) \quad|\quad \top| \boxplus_{\varrho} A\left|\boxminus_{\varrho} A\right| \bigoplus_{\varrho} A\left|\nabla_{\varrho} A\right| A \mathcal{U}_{\varrho} A^{\prime} \mid A \mathcal{S}_{\varrho} A^{\prime}
$$

2. In other words, a dyadic rational is a number of the form $n / 2^{m}$, where $n \in \mathbb{Z}$ and $m \in \mathbb{N}$. 
and $A^{+}$is given by the same grammar but without any 'non-deterministic' operators $\nabla_{\varrho}, \vartheta_{\varrho}, \mathcal{U}_{\varrho}, \mathcal{S}_{\varrho}$. The atoms $A_{1}, \ldots, A_{k}$ constitute the body of the rule, while $A^{+}$or $\perp$ its head. As usual, we assume that every variable in the head of a rule also occurs in its body.

A data instance, $\mathcal{D}$, is a finite set of facts of the form $P(\boldsymbol{c}) @ \iota$, where $P(\boldsymbol{c})$ is a ground atom (with a tuple $c$ of individual constants) and $\iota$ an interval. The fact $P(\boldsymbol{c}) @ \iota$ states that $P(\boldsymbol{c})$ holds throughout the interval $\iota$. We denote by num $(\mathcal{D})$ the set of numbers (excluding $\pm \infty$ ) that occur in $\mathcal{D}$, and by num $(\Pi, \mathcal{D})$ the set of number occurring in $\Pi$ or $\mathcal{D}$.

An interpretation, $\mathfrak{M}$, is based on a domain $\Delta \neq \emptyset$ for the individual variables and constants. For any $m$-ary predicate $P, m$-tuple $\boldsymbol{a}$ from $\Delta$, and moment of time $t \in \mathbb{R}$, the interpretation $\mathfrak{M}$ specifies whether $P$ is true on $\boldsymbol{a}$ at $t$, in which case we write $\mathfrak{M}, t=P(\boldsymbol{a})$. Let $\nu$ be an assignment of elements of $\Delta$ to the individual terms. To simplify notation, we adopt the standard name assumption according to which $\nu(c)=c$, for every individual constant $c$. We then set inductively:

$$
\begin{aligned}
& \mathfrak{M}, t \models^{\nu} P(\boldsymbol{\tau}) \quad \text { iff } \quad \mathfrak{M}, t=P(\nu(\boldsymbol{\tau})), \\
& \mathfrak{M}, t \models^{\nu}\left(\tau \neq \tau^{\prime}\right) \quad \text { iff } \quad \nu(\tau) \neq \nu\left(\tau^{\prime}\right), \\
& \mathfrak{M}, t \models^{\nu} \boxplus_{\varrho} A \quad \text { iff } \quad \mathfrak{M}, s=^{\nu} A \text { for all } s \text { with } s-t \in \varrho, \\
& \mathfrak{M}, t \models^{\nu} \boxminus_{\varrho} A \quad \text { iff } \quad \mathfrak{M}, s=^{\nu} A \text { for all } s \text { with } t-s \in \varrho, \\
& \mathfrak{M}, t \models^{\nu} \ominus_{\varrho} A \quad \text { iff } \quad \mathfrak{M}, s \models^{\nu} A \text { for some } s \text { with } s-t \in \varrho,
\end{aligned}
$$

$\mathfrak{M}, t={ }^{\nu} \ominus_{\varrho} A \quad$ iff $\quad \mathfrak{M}, s \models^{\nu} A$ for some $s$ with $t-s \in \varrho$,

$\mathfrak{M}, t \equiv^{\nu} A \mathcal{U}_{\varrho} A^{\prime} \quad$ iff $\quad \mathfrak{M}, t^{\prime} \models^{\nu} A^{\prime}$ for some $t^{\prime}$ with $t^{\prime}-t \in \varrho$ and $\mathfrak{M}, s \models^{\nu} A$ for all $s \in\left(t, t^{\prime}\right)$, $\mathfrak{M}, t \models^{\nu} A \mathcal{S}_{\varrho} A^{\prime} \quad$ iff $\quad \mathfrak{M}, t^{\prime} \models^{\nu} A^{\prime}$ for some $t^{\prime}$ with $t-t^{\prime} \in \varrho$ and $\mathfrak{M}, s \models^{\nu} A$ for all $s \in\left(t^{\prime}, t\right)$, $\mathfrak{M},\left.t\right|^{\nu} \top$, $\mathfrak{M}, t \not{ }^{\nu} \perp$.

The picture below illustrates the semantics of the 'future' operators for $\varrho=[d, e]$ :
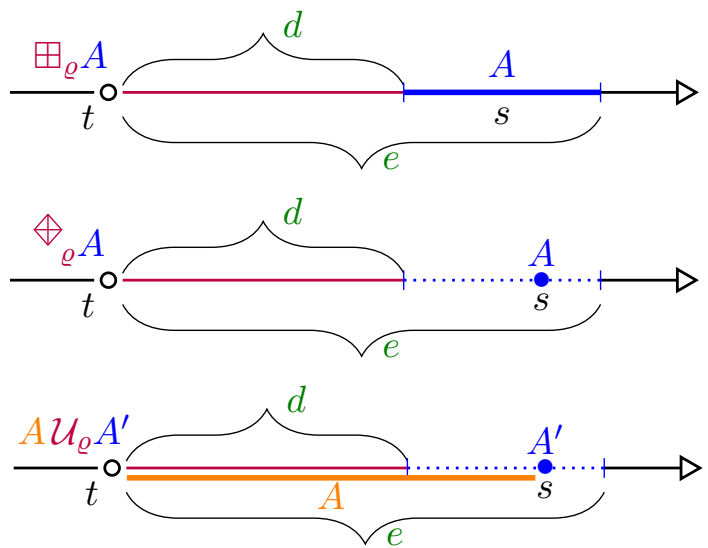

We say that $\mathfrak{M}$ satisfies a datalogMTL program $\Pi$ under an assignment $\nu$ if, for all $t \in \mathbb{R}$ and all the rules $A \leftarrow A_{1} \wedge \cdots \wedge A_{k}$ in $\Pi$, we have

$$
\mathfrak{M}, t \models^{\nu} A \text { whenever } \mathfrak{M}, t \models^{\nu} A_{i} \text { for } 1 \leq i \leq k .
$$

We call $\mathfrak{M}$ a model of $\Pi$ and $\mathcal{D}$ and write $\mathfrak{M} \models(\Pi, \mathcal{D})$ if $\mathfrak{M}$ satisfies $\Pi$ under every assignment, and $\mathfrak{M}, t \models P(\boldsymbol{c})$ for any $P(\boldsymbol{c}) @ \iota$ in $\mathcal{D}$ and any $t \in \iota$. $\Pi$ and $\mathcal{D}$ are consistent if they have a model. 
Note that ranges $\varrho$ in the temporal operators can be punctual $[r, r]$, in which case $\boxplus_{[r, r]} A$ is equivalent to $\oslash_{[r, r]} A$, and $\boxminus_{[r, r]} A$ to $\diamond_{[r, r]} A$. We also observe that $\top \mathcal{S}_{\varrho} A$ is equivalent to $\oslash_{\varrho} A$ (that is, $\mathfrak{M}, t={ }^{\nu} \top \mathcal{S}_{\varrho} A$ iff $\mathfrak{M}, t={ }^{\nu} \vartheta_{\varrho} A$ for all $\mathfrak{M}, t$ and $\nu$ ), and $\top \mathcal{U}_{\varrho} A$ is equivalent to $\uplus_{\varrho} A$.

A datalogMTL query takes the form $(\Pi, \boldsymbol{q}(\boldsymbol{v}, x))$, where $\Pi$ is a datalogMTL program and $\boldsymbol{q}(\boldsymbol{v}, x)=Q(\boldsymbol{\tau}) @ x$, for some predicate $Q, \boldsymbol{v}$ is a tuple of all individual variables occurring in the terms $\boldsymbol{\tau}$, and $x$ an interval variable. A certain answer to $(\Pi, \boldsymbol{q}(\boldsymbol{v}, x))$ over a data instance $\mathcal{D}$ is a pair $(\boldsymbol{c}, \iota)$ such that $\boldsymbol{c}$ is a tuple of constants from $\mathcal{D}$ of the same length as $\boldsymbol{v}, \iota$ an interval and, for any $t \in \iota$, any model $\mathfrak{M}$ of $\Pi$ and $\mathcal{D}$, and any assignment $\nu$ mapping $\boldsymbol{v}$ to $\boldsymbol{c}$, we have $\mathfrak{M}, t=^{\nu} Q(\boldsymbol{\tau})$. In this case, we write $\mathfrak{M}, t \models \boldsymbol{q}(\boldsymbol{c})$. If the tuple $\boldsymbol{v}$ is empty (that is, $Q(\boldsymbol{\tau})$ does not have any individual variables), then we say that $\iota$ is a certain answer to $(\Pi, \boldsymbol{q}(x))$ over $\mathcal{D}$.

Example 1. Suppose that $\Pi$ has one rule (1) and $\mathcal{D}$ consists of the facts

$$
\begin{aligned}
& \text { Turbine }(\mathrm{tb} 0) @(-\infty, \infty), \\
& \text { ActivePowerAbove1.5(tb0)@[13:00:00, 13:00:15), } \\
& \text { ActivePowerBelow0.15(tb0)@[13:00:17, 13:01:25). }
\end{aligned}
$$

Then any subinterval of the interval $[13: 01: 17,13: 01: 18)$ is a certain answer to the datalogMTL query $(\Pi$, ActivePowerTrip $(\mathrm{tb} 0) @ x)$.

Example 2. We illustrate the importance of the operators $\mathcal{S}$ (since) and $\mathcal{U}$ (until) using an example inspired by the ballet moves ontology (Raheb, Mailis, Ryzhikov, Papapetrou, \& Ioannidis, 2017). Suppose we want to say that SupportBending is a move spanning from the beginning to the end of RightAndLeftSupportLowPlace provided that it is preceded by RightAndLeftSupportMiddlePlace, which ends within $3 s$ from the beginning of the RightAndLeftSupportLowPlace, as shown below:

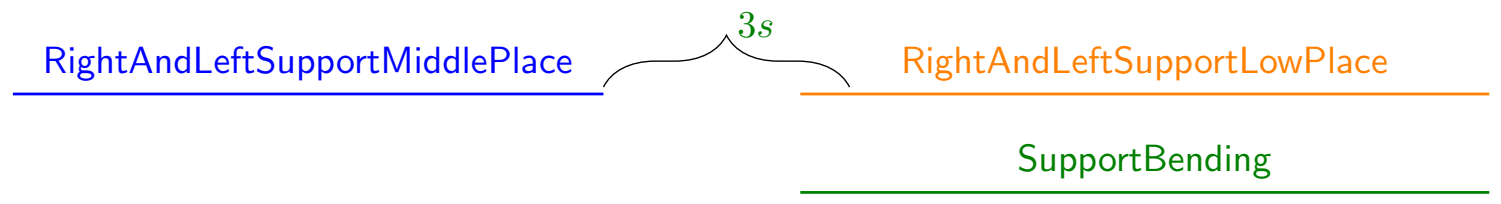

$\ominus_{[0,3 s]}$ RightAndLeftSupportMiddlePlace

We can define the SupportBending move using the following rule:

SupportBending $\leftarrow$ RightAndLeftSupportLowPlace $\mathcal{S}_{[0, \infty)}\left(\nabla_{[0,3 s]}\right.$ RightAndLeftSupportMiddlePlace).

(note that a definition of SupportBending in datalogMTL would be problematic if only the $\square$ and $\diamond$ operators were available).

By answering datalogMTL queries we understand the problem of checking whether a given pair $(\boldsymbol{c}, \iota)$ is a certain answer to a given datalogMTL query $(\Pi, \boldsymbol{q}(\boldsymbol{v}, x))$ over a given data instance $\mathcal{D}$. The consistency (or satisfiability) problem is to check whether a given datalogMTL program $\Pi$ is consistent with a given data instance $\mathcal{D}$. As usual in database theory (Vardi, 1982) and ontology-mediated query answering, we distinguish between the combined complexity and the data complexity of these problems: the former regards all the ingredients- $\Pi, \boldsymbol{q}(\boldsymbol{c}, \iota)$ and $\mathcal{D}$-as input, while the latter one assumes that $\Pi$ and $\boldsymbol{q}$ are fixed and only $\mathcal{D}$ and $(\boldsymbol{c}, \iota)$ are the input. 
Proposition 3. Answering datalogMTL queries and consistency checking are polynomially reducible to the complement of each other.

Proof. Suppose first that we want to check whether $(\boldsymbol{c}, \iota)$ is a certain answer to $(\Pi, \boldsymbol{q}(\boldsymbol{v}, x))$ over $\mathcal{D}$, where $\boldsymbol{q}(\boldsymbol{v}, x)=Q(\boldsymbol{\tau}) @ x$ and $\iota=\left[-t_{1}, t_{2}\right), t_{1}, t_{2} \in \mathbb{Q}_{2}^{\geq 0}$; other types of $\iota$ are considered analogously. Consider the following program $\Pi^{\prime}$ and data instance $\mathcal{D}^{\prime}$ :

$$
\begin{aligned}
& \Pi^{\prime}=\Pi \cup\left\{\perp \leftarrow P(\boldsymbol{v}) \wedge \boxminus_{\left[0, t_{1}\right]} Q(\boldsymbol{v}) \wedge \boxplus_{\left(0, t_{2}\right)} Q(\boldsymbol{v})\right\}, \\
& \mathcal{D}^{\prime}=\mathcal{D} \cup\{P(\boldsymbol{c}) @[0,0]\},
\end{aligned}
$$

where $P$ is a fresh predicate. It is readily seen that $(\boldsymbol{c}, \iota)$ is a certain answer to $(\Pi, \boldsymbol{q}(\boldsymbol{v}, x))$ over $\mathcal{D}$ iff $\Pi^{\prime}$ is not consistent with $\mathcal{D}^{\prime}$. Conversely, $\Pi$ and $\mathcal{D}$ are consistent iff $[0,0]$ is not a certain answer to $(\Pi, P @ x)$ over $\mathcal{D}$, where $P$ is a fresh 0 -ary predicate, that is, a propositional variable.

We conclude this section by reminding the reader that, over the integer numbers $(\mathbb{Z},<), M T L$ is as expressive as the linear temporal logic LTL with the operators $\bigcirc_{F}$ (at the next moment), $\mathcal{U}$ (until), $\square_{F}$ (always in the future), $\diamond_{F}$ (some time in the future) and their past counterparts $\bigcirc_{P}, \mathcal{S}$, $\square_{P}$ and $\diamond_{P}$. For example, the $L T L$-formula $\bigcirc_{F} A$ is equivalent to $\oslash_{[1,1]} A$ and $A \mathcal{U} B$ under the irreflexive semantics to $A \mathcal{U}_{(0, \infty)} B$; conversely, $\oslash_{[2,3]} A$ is clearly equivalent to the $L T L$-formula $\bigcirc_{F} \bigcirc_{F} A \vee \bigcirc_{F} \bigcirc_{F} \bigcirc_{F} A$. However, MTL operators are more succinct, which explains why MTLsatisfiability over $(\mathbb{Z},<)$ is EXPSPACE-complete (Alur \& Henzinger, 1993; Furia \& Spoletini, 2008) whereas LTL-satisfiability is PSPACE-complete (Sistla \& Clarke, 1985).

In the next section, we show that consistency checking for datalogMTL programs is EXPSPACEcomplete for combined complexity. It follows from Proposition 3 that answering datalogMTL queries is EXPSPACE-complete as well. On the other hand, we also prove that answering propositional datalogMTL queries is P-hard for data complexity, and that the extension of datalogMTL with $\ominus$ in the head of rules leads to undecidability.

\section{Complexity of Answering DatalogMTL Queries}

Observe first that every datalog $M T L$ program $\Pi$ can be transformed (using polynomially-many fresh predicates) to a datalogMTL program in normal form that only contains rules such as

$$
\begin{array}{lll}
P(\boldsymbol{\tau}) \leftarrow \bigwedge_{i \in I} P_{i}\left(\boldsymbol{\tau}_{i}\right), & \perp \leftarrow \bigwedge_{i \in I} P_{i}\left(\boldsymbol{\tau}_{i}\right), \\
P(\boldsymbol{\tau}) \leftarrow P_{1}\left(\boldsymbol{\tau}_{1}\right) \mathcal{S}_{\varrho} P_{2}\left(\boldsymbol{\tau}_{2}\right), & & P(\boldsymbol{\tau}) \leftarrow P_{1}\left(\boldsymbol{\tau}_{1}\right) \mathcal{U}_{\varrho} P_{2}\left(\boldsymbol{\tau}_{2}\right), \\
P(\boldsymbol{\tau}) \leftarrow \boxminus_{\varrho} P_{1}\left(\boldsymbol{\tau}_{1}\right), & & P(\boldsymbol{\tau}) \leftarrow \boxplus_{\varrho} P_{1}\left(\boldsymbol{\tau}_{1}\right),
\end{array}
$$

and gives the same certain answers as $\Pi$ over any data instance. (In particular, datalogMTL programs in normal form do not contain occurrences of the diamond operators.) For example, we can replace the rule $\boxplus_{\varrho^{\prime}} P(\boldsymbol{\tau}) \leftarrow P_{1}\left(\boldsymbol{\tau}_{1}\right) \wedge \boxminus_{\varrho} P_{2}\left(\boldsymbol{\tau}_{2}\right)$ in $\Pi$ with three rules

$$
\begin{aligned}
& P^{\prime}(\boldsymbol{\tau}) \leftarrow P_{1}\left(\boldsymbol{\tau}_{1}\right) \wedge P_{2}^{\prime}\left(\boldsymbol{\tau}_{2}\right), \\
& P_{2}^{\prime}\left(\boldsymbol{\tau}_{2}\right) \leftarrow \boxminus_{\varrho} P_{2}\left(\boldsymbol{\tau}_{2}\right), \\
& P(\boldsymbol{\tau}) \leftarrow \top \mathcal{S}_{\varrho^{\prime}} P^{\prime}(\boldsymbol{\tau}),
\end{aligned}
$$


where $P^{\prime}$ is a fresh predicate of the same arity as $P$ and $P_{2}^{\prime}$ a fresh predicate of the same arity as $P_{2}$. Moreover, we can only consider those programs and data instances where intervals take one of the following two forms:

- $\left(t_{1}, t_{2}\right)$ with $t_{1}, t_{2} \in \mathbb{Q}_{2} \cup\{-\infty, \infty\}$,

- $[t, t]$ with $t \in \mathbb{Q}_{2}$; such intervals are called punctual.

For example, a data instance $\mathcal{D}=\mathcal{D}^{\prime} \cup\left\{P(\boldsymbol{c}) @\left(t_{1}, t_{2}\right]\right\}$ is equivalent to the data instance

$$
\mathcal{D}=\mathcal{D}^{\prime} \cup\left\{P(\boldsymbol{c}) @\left(t_{1}, t_{2}\right), P(\boldsymbol{c}) @\left[t_{2}, t_{2}\right]\right\}
$$

in the sense that is gives the same certain answers as $\mathcal{D}$, the rule $P(v) \leftarrow \boxminus_{\left(r_{1}, r_{2}\right]} P^{\prime}(v)$ is equivalent to $P(v) \leftarrow \boxminus_{\left(r_{1}, r_{2}\right)} P^{\prime}(v) \wedge \boxminus_{\left[r_{2}, r_{2}\right]} P^{\prime}(v)$, whereas the rule $P(v) \leftarrow P_{1}(v) \mathcal{U}_{\left(r_{1}, r_{2}\right]} P_{2}(v)$ is equivalent to the pair of rules

$$
P(v) \leftarrow P_{1}(v) \mathcal{U}_{\left(r_{1}, r_{2}\right)} P_{2}(v), \quad P(v) \leftarrow P_{1}(v) \mathcal{U}_{\left[r_{2}, r_{2}\right]} P_{2}(v) .
$$

We use the following notations. We assume that $\langle$ is one of ( and [, while $\rangle$ is one of ) and ]. Given an interval $\iota=\left\langle\iota_{b}, \iota_{e}\right\rangle$ and a range $\varrho$, we set

$$
\iota+{ }^{o} \varrho=\left\{\begin{array}{ll}
\left\langle\iota_{b}+r, \iota_{e}+r\right\rangle, & \text { if } \varrho=[r, r], \\
\left(\iota_{b}+r_{1}, \iota_{e}+r_{2}\right), & \text { if } \varrho=\left(r_{1}, r_{2}\right),
\end{array} \quad \iota-^{o} \varrho= \begin{cases}\left\langle\iota_{b}-r, \iota_{e}-r\right\rangle, & \text { if } \varrho=[r, r], \\
\left(\iota_{b}-r_{2}, \iota_{e}-r_{1}\right), & \text { if } \varrho=\left(r_{1}, r_{2}\right) .\end{cases}\right.
$$

In other words, $\iota+^{o} \varrho=\{t+k \mid t \in \iota$ and $k \in \varrho\}$ and $\iota-^{o} \varrho=\{t-k \mid t \in \iota$ and $k \in \varrho\}$. We also set

$$
\begin{aligned}
& \iota-{ }^{c} \varrho=\left\{\begin{array}{lll}
\left\langle\iota_{b}-r, \iota_{e}-r\right\rangle, & \text { if } \varrho=[r, r], \\
{\left[\iota_{b}-r_{1}, \iota_{e}-r_{2}\right],} & \text { if } \varrho=\left(r_{1}, r_{2}\right), & r_{2}, \iota_{e} \in \mathbb{Q}_{2}, \\
{\left[\iota_{b}-r_{1}, \infty\right),} & \text { if } \varrho=\left(r_{1}, r_{2}\right), & r_{2}=\infty \text { or } \iota_{e}=\infty,
\end{array}\right. \\
& \iota+{ }^{c} \varrho=\left\{\begin{array}{lll}
\left\langle\iota_{b}+r, \iota_{e}+r\right\rangle, & \text { if } \varrho=[r, r], \\
{\left[\iota_{b}+r_{2}, \iota_{e}+r_{1}\right],} & \text { if } \varrho=\left(r_{1}, r_{2}\right), & r_{2}, \iota_{b} \in \mathbb{Q}_{2}, \\
\left(-\infty, \iota_{e}+r_{1}\right], & \text { if } \varrho=\left(r_{1}, r_{2}\right), & r_{2}=\infty \text { or } \iota_{b}=-\infty .
\end{array}\right.
\end{aligned}
$$

We assume that $\iota-{ }^{c} \varrho$ and $\iota+{ }^{c} \varrho$ are only defined if $r_{2}-r_{1} \leq \iota_{e}-\iota_{b}$, in which case we write $\varrho \sqsubseteq \iota$. Thus, $\iota-{ }^{c} \varrho$ is defined if there is $t^{\prime}$ such that $t^{\prime}+k \in \iota$, for all $k \in \varrho$. Symmetrically, $\iota+{ }^{c} \varrho$ is defined if there is $t^{\prime}$ such that $t^{\prime}-k \in \iota$. The picture below illustrates the intuition behind $\iota+^{o} \varrho$ and $\iota+{ }^{c} \varrho$, for non-punctual $\varrho$, and the difference between them:

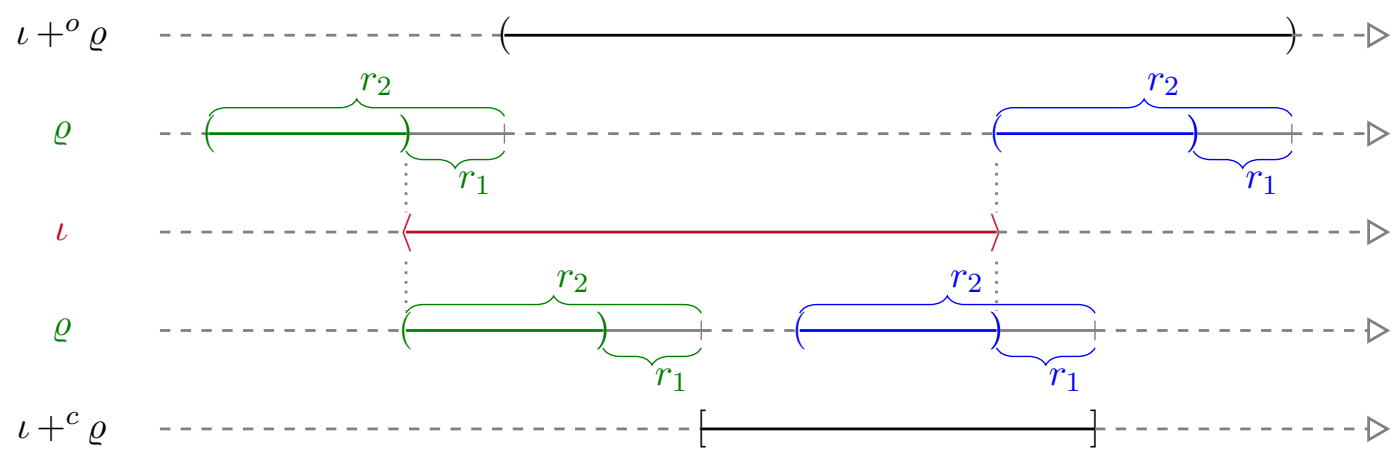


Furthermore, we write

- $\bigcap_{i \in I} \iota_{i} \neq \emptyset$ to say that the intersection of the intervals $\iota_{i}$, for $i \in I$, is non-empty;

- $\bigcap_{i \in I} \iota_{i}$ for the intersection of the intervals $\iota_{i}$ provided that $\bigcap_{i \in I} \iota_{i} \neq \emptyset$; otherwise $\bigcap_{i \in I} \iota_{i}$ is undefined;

- $\bigcup_{i \in I} \iota_{i}$ for the union of the intervals $\iota_{i}$ provided that $\bigcup_{i \in I} \iota_{i}$ is a single interval; otherwise $\bigcup_{i \in I} \iota_{i}$ is undefined;

- $\iota^{c}$ for the closure of an interval $\iota$, that is $\iota^{c}=\left[\iota_{b}, \iota_{e}\right]$ for any $\iota=\left\langle\iota_{b}, \iota_{e}\right\rangle$.

Suppose now that we are given a datalogMTL program $\Pi$ (in normal form) and a data instance $\mathcal{D}$. We define a (possibly infinite) set $\mathfrak{C}_{\Pi, \mathcal{D}}$ of atoms of the form $P(\boldsymbol{c}) @ \iota$ or $\perp @_{\iota}$ that contains all answers to datalogMTL queries with $\Pi$ over $\mathcal{D}$. The construction is essentially the standard chase procedure from database theory (Abiteboul, Hull, \& Vianu, 1995) adapted to time intervals and the temporal operators by mimicking their semantics. The only new chase rule is coalescing (coal) that merges-possibly infinitely-many-smaller intervals into the lager one they cover. Because of this rule, our chase construction requires transfinite recursion; see also the work by Bresolin, Kurucz, Muñoz-Velasco, Ryzhikov, Sciavicco, and Zakharyaschev (2017) and Artale et al. (2015).

Let $\mathcal{C}$ be some set of atoms of the form $P(\boldsymbol{c}) @ \iota$ or $\perp @ \iota$ from $\Pi$ and $\mathcal{D}$. Denote by $\operatorname{cl}(\mathcal{C})$ the result of applying exhaustively and non-recursively the following rules to $\mathcal{C}$ :

(coal) if $P(\boldsymbol{c}) @ \iota_{i} \in \mathcal{C}$, for all $i \in I$ with a possibly infinite set $I$, and $\bigcup_{i \in I} \iota_{i}$ is defined, then we add $P(\boldsymbol{c}) @ \bigcup_{i \in I} \iota_{i}$ to $\mathcal{C}$;

(horn) if $P(\boldsymbol{c}) \leftarrow \bigwedge_{i \in I} P_{i}\left(\boldsymbol{c}_{i}\right)$ is an instance of a rule in $\Pi$ with all $P_{i}\left(\boldsymbol{c}_{i}\right) @_{\iota_{i}}$ in $\mathcal{C}$ and $\bigcap_{i \in I} \iota_{i} \neq \emptyset$, then we add $P(\boldsymbol{c}) @ \bigcap_{i \in I} \iota_{i}$ to $\mathcal{C}$; if $\perp \leftarrow \bigwedge_{i \in I} P_{i}\left(\boldsymbol{c}_{i}\right)$ is an instance of a rule in $\Pi$, then we add $\perp @ \bigcap_{i \in I} \iota_{i}$ to $\mathcal{C}$;

$\left(\mathcal{S}_{\varrho}\right)$ if $P(\boldsymbol{c}) \leftarrow P_{1}\left(\boldsymbol{c}_{1}\right) \mathcal{S}_{\varrho} P_{2}\left(\boldsymbol{c}_{2}\right)$ is an instance of a rule in $\Pi$ with $P_{i}\left(\boldsymbol{c}_{i}\right) @ \iota_{i} \in \mathcal{C}$ for $i \in\{1,2\}$, $\iota_{1}^{c} \cap \iota_{2} \neq \emptyset$, and $\left(\left(\iota_{1}^{c} \cap \iota_{2}\right)+{ }^{o} \varrho\right) \cap \iota_{1}^{c} \neq \emptyset$, then we add $P(\boldsymbol{c}) @\left(\left(\iota_{1}^{c} \cap \iota_{2}\right)+{ }^{o} \varrho\right) \cap \iota_{1}^{c}$ to $\mathcal{C}$; see the picture below, where $\varrho=\left(r_{1}, r_{2}\right)$;

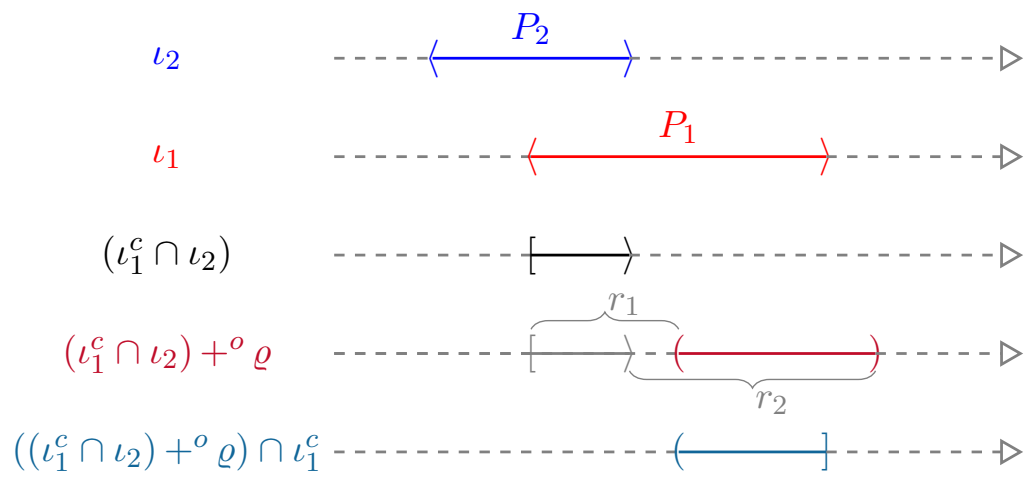

$\left(\boxplus_{\varrho}\right)$ if $P(\boldsymbol{c}) \leftarrow \boxplus_{\varrho} P_{1}\left(\boldsymbol{c}_{1}\right)$ is an instance of a rule in $\Pi$ with $P_{1}\left(\boldsymbol{c}_{1}\right) @ \iota \in \mathcal{C}$ and $\varrho \sqsubseteq \iota$, then we add $P(\boldsymbol{c}) @\left(\iota-{ }^{c} \varrho\right)$ to $\mathcal{C}$; 
$\left(\mathcal{U}_{\varrho}\right)$ if $P(\boldsymbol{c}) \leftarrow P_{1}\left(\boldsymbol{c}_{1}\right) \mathcal{U}_{\varrho} P_{2}\left(\boldsymbol{c}_{2}\right)$ is an instance of a rule in $\Pi$ with $P_{i}\left(\boldsymbol{c}_{i}\right) @ \iota_{i} \in \mathcal{C}, \iota_{1}^{c} \cap \iota_{2} \neq \emptyset$ and $\left(\left(\iota_{1}^{c} \cap \iota_{2}\right)-{ }^{o} \varrho\right) \cap \iota_{1}^{c} \neq \emptyset$, then we add $P(\boldsymbol{c}) @\left(\left(\iota_{1}^{c} \cap \iota_{2}\right)-{ }^{o} \varrho\right) \cap \iota_{1}^{c}$ to $\mathcal{C}$;

$\left(\Xi_{\varrho}\right)$ if $P(\boldsymbol{c}) \leftarrow \boxminus_{\varrho} P_{1}\left(\boldsymbol{c}_{1}\right)$ is an instance of a rule in $\Pi$ with $P_{1}\left(\boldsymbol{c}_{1}\right) @ \iota \in \mathcal{C}$ and $\varrho \sqsubseteq \iota$, then we add $P(c) @\left(\iota+{ }^{c} \varrho\right)$ to $\mathcal{C}$.

We set $\operatorname{cl}^{0}(\mathcal{D})=\mathcal{D} \cup\{\top(-\infty, \infty)\}$ and, for any successor ordinal $\xi+1$ and limit ordinal $\zeta$,

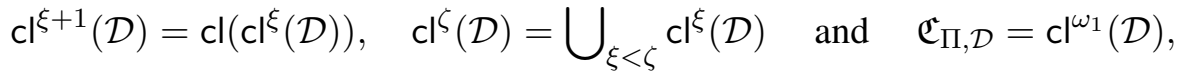

where $\omega_{1}$ is the first uncountable ordinal (as $\mathrm{cl}^{\omega_{1}}(\mathcal{D})$ is countable, there is an ordinal $\alpha<\omega_{1}$ such that $\mathrm{cl}^{\alpha}(\mathcal{D})=\mathrm{cl}^{\beta}(\mathcal{D})$, for all $\beta \geq \alpha$ ). We regard $\mathfrak{C}_{\Pi, \mathcal{D}}$ as both a set of atoms of the form $P(\boldsymbol{c}) @ \iota$ or $\perp @ \iota$ and an interpretation where, for any $t \in \mathbb{R}$, any $P$ (different from $\perp$ ), and any tuple $c$ of individual constants, we have $\mathfrak{C}_{\Pi, \mathcal{D}}, t=P(\boldsymbol{c})$ iff $P(\boldsymbol{c}) @_{\iota} \in \mathfrak{C}_{\Pi, \mathcal{D}}$ and $t \in \iota$. The domain of $\mathfrak{C}_{\Pi, \mathcal{D}}$ is the set ind $(\mathcal{D}) \cup$ ind $(\Pi)$ that comprises the individual constants occurring in $\mathcal{D}$ and $\Pi$.

We illustrate the definition above by a simple example:

Example 4. Let $\Pi$ have two rules $P \leftarrow \boxminus_{[1,1]} P$ and $Q \leftarrow \boxplus_{(0, \infty)} P$, and let $\mathcal{D}=\{P(0,1]\}$. The first $\omega$ steps of the construction of $\mathfrak{C}_{\Pi, \mathcal{D}}$ will produce, using the rules $\left(\boxminus_{\varrho}\right)$ and (coal), the atoms $P(n, n+1]$ and $P(0, n+1]$, for $n<\omega$. In the step $\omega+1$, (coal) will give $P(0, \infty)$ and then $\left(\boxplus_{\varrho}\right)$ will return $Q @[0, \infty)$.

Lemma 5. Let $\Pi$ be a datalogMTL program and $\mathcal{D}$ a data instance. Then, for any predicate symbol $P$ from $\Pi$ and $\mathcal{D}$, any tuple $c$ of constants from $\mathcal{D}$ and $\Pi$, and any interval $\iota$,

(i) $P(\boldsymbol{c}) @_{\iota} \in \mathfrak{C}_{\Pi, \mathcal{D}}$ implies $\mathfrak{M}, t=P(\boldsymbol{c})$, for all $t \in \iota$ and all models $\mathfrak{M}$ of $\Pi$ and $\mathcal{D}$;

(ii) if $\perp @ \iota \notin \mathfrak{C}_{\Pi, \mathcal{D}}$ for any $\iota$, then $\mathfrak{C}_{\Pi, \mathcal{D}} \models(\Pi, \mathcal{D})$; otherwise, $\Pi$ and $\mathcal{D}$ are inconsistent.

Proof. (i) Suppose that $\mathfrak{M}$ is a model of $\Pi$ and $\mathcal{D}$, and that $P(\boldsymbol{c}) @ \iota \in \mathfrak{C}_{\Pi, \mathcal{D}}$. Let $\xi$ be the smallest ordinal such that $P(\boldsymbol{c}) @ \iota \in \mathrm{cl}^{\xi}(\mathcal{D})$. We show that $\mathfrak{M}, t=P(\boldsymbol{c})$ for all $t \in \iota$ by induction of $\xi$. If $\xi=0$, then $P(\boldsymbol{c}) @ \iota \in \mathcal{D}$, and since $\mathfrak{M}$ satisfies every assertion in $\mathcal{D}$, we are done. If $\xi=\xi^{\prime}+1$ then $P(\boldsymbol{c}) @ \iota$ was obtained from $\mathrm{cl}^{\xi^{\prime}}(\mathcal{D})$ by applying one of the construction rules for $\mathfrak{C}_{\Pi, \mathcal{D}}$. Suppose $P(\boldsymbol{c}) @ \iota$ is $P(\boldsymbol{c}) @ \bigcup_{i \in I} \iota_{i}$ obtained by (coal). By the induction hypothesis, $\mathfrak{M}, t \models P(\boldsymbol{c})$ for all $t \in \iota_{i}$ and $i \in I$. Clearly, $\mathfrak{M}, t=P(\boldsymbol{c})$ for all $t \in \bigcup_{i \in I} \iota_{i}$, and so for all $t \in \iota$. The case of (horn) is similar (with intersection in place of union).

Suppose $P(\boldsymbol{c}) @ \iota$ is obtained by $\left(\mathcal{S}_{\varrho}\right)$ from $P_{i}\left(\boldsymbol{c}_{i}\right) @ \iota_{i}, i \in\{1,2\}$. By the induction hypothesis, $\mathfrak{M}, t \models P_{i}\left(\boldsymbol{c}_{i}\right)$ for every $t \in \iota_{i}$. Take an arbitrary $t \in\left(\left(\iota_{1}^{c} \cap \iota_{2}\right)+{ }^{o} \varrho\right) \cap \iota_{1}^{c}$. Then there exists $t^{\prime} \in \iota_{1}^{c} \cap \iota_{2}$ such that $t-t^{\prime} \in \varrho$ and $\mathfrak{M}, t=P_{2}\left(\boldsymbol{c}_{2}\right)$. Moreover, we have $\mathfrak{M}, s \models P_{1}\left(\boldsymbol{c}_{1}\right)$ for all $s \in\left(t^{\prime}, t\right)$. Therefore, $\mathfrak{M}, t=P_{1}\left(\boldsymbol{c}_{1}\right) \mathcal{S} P_{2}\left(\boldsymbol{c}_{2}\right)$. If $P(\boldsymbol{c}) @_{\iota}$ is obtained by $\left(\boxplus_{\varrho}\right)$ from $P_{1}\left(\boldsymbol{c}_{1}\right) @ \iota$, the proof is analogous by considering $t \in \iota-{ }^{c} \varrho$. The remaining rules are treated similarly.

(ii) Suppose $\perp @ \iota \notin \mathfrak{C}_{\Pi, \mathcal{D}}$ for any $\iota$. By definition, $\mathcal{D} \subseteq \mathfrak{C}_{\Pi, \mathcal{D}}$, and so $\mathfrak{C}_{\Pi, \mathcal{D}}=P(\boldsymbol{c}) @ \iota$ for every $P(\boldsymbol{c}) @_{\iota} \in \mathcal{D}$. To show that all the rules in $\Pi$ are satisfied by $\mathfrak{C}_{\Pi, \mathcal{D}}$, we take an assignment $\nu$, a rule $P(\boldsymbol{\tau}) \leftarrow \bigwedge_{i \in I} P_{i}\left(\boldsymbol{\tau}_{i}\right)$ from $\Pi$, and suppose that $\mathfrak{C}_{\Pi, \mathcal{D}}, t \models^{\nu} P_{i}\left(\boldsymbol{\tau}_{i}\right)$, for all $i \in I$. By the definition of $\mathfrak{C}_{\Pi, \mathcal{D}}$, it follows that $\mathfrak{C}_{\Pi, \mathcal{D}}, t \models P_{i}\left(\nu\left(\boldsymbol{\tau}_{i}\right)\right)$ and $P_{i}\left(\nu\left(\boldsymbol{\tau}_{i}\right)\right) \in \mathfrak{C}_{\Pi, \mathcal{D}}$, for some $\iota_{i} \ni t$. Moreover, there are ordinals $\xi_{i}, i \in I$, such that $P_{i}\left(\nu\left(\boldsymbol{\tau}_{i}\right)\right) @_{\iota_{i}} \in \mathrm{cl}^{\xi_{i}}(\mathcal{D})$. By the rule (horn), we then have $P(\nu(\boldsymbol{\tau})) @ \bigcap_{i \in I} \iota_{i} \in \mathrm{cl}^{\max \left\{\xi_{i} \mid i \in I\right\}+1}(\mathcal{D})$, from which $P(\nu(\boldsymbol{\tau})) @ \bigcap_{i \in I} \iota_{i} \in \mathfrak{C}_{\Pi, \mathcal{D}}$, and so $\mathfrak{C}_{\Pi, \mathcal{D}}, t \models P(\nu(\boldsymbol{\tau}))$. Now, consider a rule $\perp \leftarrow \bigwedge_{i \in I} P_{i}\left(\boldsymbol{\tau}_{i}\right)$ and suppose that $\mathfrak{C}_{\Pi, \mathcal{D}}, t \models{ }^{\nu} P_{i}\left(\boldsymbol{\tau}_{i}\right)$, for 
all $i \in I$. By the argument above, we then should have $\perp @ \bigcap_{i \in I} \iota_{i} \in \mathfrak{C}_{\Pi, \mathcal{D}}$, which is a contradiction. For a rule $P(\boldsymbol{\tau}) \leftarrow P_{1}\left(\boldsymbol{\tau}_{1}\right) \mathcal{S}_{\varrho} P_{2}\left(\boldsymbol{\tau}_{2}\right)$, take an arbitrary $t$ and suppose that $\mathfrak{C}_{\Pi, \mathcal{D}}, t_{2} \models{ }^{\nu} P_{2}\left(\boldsymbol{\tau}_{2}\right)$ for some $t_{2}$ with $t-t_{2} \in \varrho$ and $\mathfrak{C}_{\Pi, \mathcal{D}}, t_{1} \mid=^{\nu} P_{1}\left(\boldsymbol{\tau}_{1}\right)$ for all $t_{2} \in\left(t_{2}, t\right)$. By the construction of $\mathfrak{C}_{\Pi, \mathcal{D}}$, it follows that $P_{2}\left(\nu\left(\boldsymbol{\tau}_{2}\right)\right) @ \iota_{2} \in \mathfrak{C}_{\Pi, \mathcal{D}}$ for some $\iota_{2} \ni t_{2}$. Moreover, there are finitely many intervals $\iota_{i}^{\prime}, i \in I$, such that $\left(t_{2}, t\right) \subseteq \bigcup_{i \in I} \iota_{i}^{\prime}$ and $P_{1}\left(\nu\left(\tau_{1}\right)\right) @ \iota_{i}^{\prime} \in \mathfrak{C}_{\Pi, \mathcal{D}}$. By the rule (coal), $P_{1}\left(\nu\left(\boldsymbol{\tau}_{1}\right)\right) @ \iota_{1} \in \mathfrak{C}_{\Pi, \mathcal{D}}$ for $\iota_{1}=\bigcup_{i \in I} \iota_{i}^{\prime}$. It follows then that $t_{2}, t \in \iota_{1}^{c}$, and so $\iota_{1}^{c} \cap \iota_{2} \neq \emptyset$ and $t \in\left(\left(\iota_{1}^{c} \cap \iota_{2}\right)+{ }^{o} \varrho\right) \cap \iota_{1}^{c}$. Thus, by the rule $\left(\mathcal{S}_{\varrho}\right)$, we have $P(\nu(\boldsymbol{\tau})) @\left(\left(\iota_{1}^{c} \cap \iota_{2}\right)+{ }^{o} \varrho\right) \cap \iota_{1}^{c} \in \mathfrak{C}_{\Pi, \mathcal{D}}$. Therefore, $\mathfrak{C}_{\Pi, \mathcal{D}}, t \models^{\nu} P(\boldsymbol{\tau})$. The remaining rules are considered in the same manner.

That $\perp @ \iota \in \mathfrak{C}_{\Pi, \mathcal{D}}$, for some $\iota$, implies inconsistency of $\mathcal{D}$ and $\Pi$ follows from $(i)$.

If $\perp @_{\iota} \notin \mathfrak{C}_{\Pi, \mathcal{D}}$, we call $\mathfrak{C}_{\Pi, \mathcal{D}}$ the canonical (or minimal) model of $\Pi$ and $\mathcal{D}$. We now establish an important property of $\mathfrak{C}_{\Pi, \mathcal{D}}$ that will allow us to reduce consistency checking for datalogMTL programs and data to the satisfiability problem for formulas in the linear temporal logic LTL over $(\mathbb{Z},<)$.

Recall that the greatest common divisor of a finite set $N \subseteq \mathbb{Q}$ (at least one of which is not 0 ) is the largest number $\operatorname{gcd}(N)>0$ such that every $n \in N$ is divisible by $\operatorname{gcd}(N)$ (in the sense that $n / \operatorname{gcd}(N) \in \mathbb{Z})$. It is known that $\operatorname{gcd}(N)$ always exists and $\operatorname{gcd}(N) \leq \prod_{n \in N}|n|$. It is easy to see that, for any a finite set $N \subseteq \mathbb{Q}_{2}$ (at least one of which is not 0 ), we have $\operatorname{gcd}(N)=2^{m}$, where $m$ is the maximal natural number such that $n / 2^{m} \in N$ is an irreducible fraction. Thus, $\operatorname{gcd}(N)$ can be computed and stored using space polynomial in $|N|$ (the size of the binary encoding of $N$ ). To make further definitions simpler, it will be convenient to assume that $\operatorname{gcd}(N)=1$ if $N=\{0\}$.

Given a datalogMTL program $\Pi$ and a data instance $\mathcal{D}$, we take $d=\operatorname{gcd}(\operatorname{num}(\Pi, \mathcal{D}))$. Denote by $\sec _{\Pi, \mathcal{D}}$ the set of all the intervals of the form $[k d, k d]$ and $((k-1) d, k d)$, for $k \in \mathbb{Z}$. Clearly, $\sec _{\Pi, \mathcal{D}}$ is a partition of $\mathbb{Q}_{2}$. We represent $\sec _{\Pi, \mathcal{D}}$ as

$$
\sec _{\Pi, \mathcal{D}}=\left\{\ldots, \sigma_{-3}, \sigma_{-2}, \sigma_{-1}, \sigma_{0}, \sigma_{1}, \sigma_{2}, \sigma_{3}, \ldots\right\},
$$

where $\sigma_{0}=[0,0], \sigma_{1}=(0, d), \sigma_{2}=[d, d], \sigma_{3}=(d, 2 d), \sigma_{-1}=(-d, 0)$, etc. Thus, $\sigma_{i}$ is punctual if $i$ is even and non-punctual if $i$ is odd. We refer to the $\sigma_{i}$ as sections of $\sec _{\Pi, \mathcal{D}}$.

Lemma 6. For every atom $P(\boldsymbol{c})$ and every $\sigma \in \sec _{\Pi, \mathcal{D}}$, we either have $\mathfrak{C}_{\Pi, \mathcal{D}}, t \models P(\boldsymbol{c})$ for all $t \in \sigma$, or $\mathfrak{C}_{\Pi, \mathcal{D}}, t \not \models P(\boldsymbol{c})$ for all $t \in \sigma$.

Proof. It suffices to show that every interval $\iota$ such that $P(\boldsymbol{c}) @ \iota \in \mathfrak{C}_{\Pi, \mathcal{D}}$ takes one of the following forms: $(-\infty, \infty),\langle d k, \infty),(-\infty, d k\rangle,\left\langle d k, d k^{\prime}\right\rangle$, where $k, k^{\prime} \in \mathbb{Z}$. This can readily be done by induction on the construction of $\mathfrak{C}_{\Pi, \mathcal{D}}$. Indeed, when applied to a set of atoms of this form, the operator $\mathrm{Cl}$ also results in a set of such atoms.

Our aim now is to encode the structure of $\mathfrak{C}_{\Pi, \mathcal{D}}$ given by Lemma 6 by means of an $L T L$-formula $\varphi_{\Pi, \mathcal{D}}$ that is satisfiable over $(\mathbb{Z},<)$ iff $\Pi$ and $\mathcal{D}$ are consistent. The $L T L$-formula $\varphi_{\Pi, \mathcal{D}}$ contains propositional variables of the form $P^{c}$, where $P$ is a predicate symbol from $\Pi$ and $\mathcal{D}$ of arity $m$ and $c$ an $m$-tuple of individual constants from $\mathcal{D}$ and $\Pi$, as well as two additional propositional variables odd and even. We define $\varphi_{\Pi, \mathcal{D}}$ as a conjunction of the following clauses, where $\nu$ is any assignment of the individual constants from $\mathcal{D}$ and $\Pi$ to the terms in $\Pi$, and $\square \psi$ is a shorthand for $\square_{P} \varphi \wedge \varphi \wedge \square_{F} \varphi$ :

- even $\wedge \square\left(\right.$ even $\leftrightarrow \bigcirc_{F}$ odd $) \wedge \square\left(\right.$ odd $\leftrightarrow \bigcirc_{F}$ even $)$; 
$-\square\left(P^{\nu(\boldsymbol{\tau})} \leftarrow \bigwedge_{i \in I} P_{i}^{\nu\left(\boldsymbol{\tau}_{i}\right)}\right)$, for every rule $P(\boldsymbol{\tau}) \leftarrow \bigwedge_{i \in I} P_{i}\left(\boldsymbol{\tau}_{i}\right)$ in $\Pi$;

$-\square\left(\perp \leftarrow \bigwedge_{i \in I} P_{i}^{\nu\left(\tau_{i}\right)}\right)$, for every rule $\perp \leftarrow \bigwedge_{i \in I} P_{i}\left(\boldsymbol{\tau}_{i}\right)$ in $\Pi$;

- for every rule $P(\boldsymbol{\tau}) \leftarrow P_{1}\left(\boldsymbol{\tau}_{1}\right) \mathcal{S}_{\varrho} P_{2}\left(\boldsymbol{\tau}_{2}\right)$ in $\Pi$ with $\varrho=[r, r]$, we require two clauses:

$$
\begin{aligned}
& \square\left(P^{\nu(\boldsymbol{\tau})} \leftarrow \text { even } \wedge \bigcirc^{-2 r / d} P_{2}^{\nu\left(\boldsymbol{\tau}_{2}\right)} \wedge \bigwedge_{-2 r / d<j<0} \bigcirc^{j} P_{1}^{\nu\left(\boldsymbol{\tau}_{1}\right)}\right), \\
& \square\left(P^{\nu(\boldsymbol{\tau})} \leftarrow \text { odd } \wedge \bigcirc^{-2 r / d} P_{2}^{\nu\left(\boldsymbol{\tau}_{2}\right)} \wedge \bigwedge_{-2 r / d \leq j \leq 0} \bigcirc^{j} P_{1}^{\nu\left(\boldsymbol{\tau}_{1}\right)}\right),
\end{aligned}
$$

where $\bigcirc^{n} \varphi=\underbrace{\bigcirc_{F} \ldots \bigcirc_{F}}_{n} \varphi$ if $n>0, \bigcirc^{0} \varphi=\varphi$, and $\bigcirc^{n} \varphi=\underbrace{\bigcirc_{P} \ldots \bigcirc_{P}}_{|n|} \varphi$ if $n<0$;

- for every rule $P(\boldsymbol{\tau}) \leftarrow P_{1}\left(\boldsymbol{\tau}_{1}\right) \mathcal{S}_{\varrho} P_{2}\left(\boldsymbol{\tau}_{2}\right)$ in $\Pi$ with $\varrho=\left(r_{1}, r_{2}\right)$, we require four clauses:

$$
\begin{gathered}
\square\left(P^{\nu(\boldsymbol{\tau})} \leftarrow \text { even } \wedge \bigvee_{-2 r_{2} / d<k<-2 r_{1} / d}\left(\bigcirc^{k} P_{2}^{\nu\left(\boldsymbol{\tau}_{2}\right)} \wedge \bigcirc^{k} \text { even } \wedge \bigwedge_{k<j<0} \bigcirc^{j} P_{1}^{\nu\left(\boldsymbol{\tau}_{1}\right)}\right),\right. \\
\square\left(P^{\nu(\boldsymbol{\tau})} \leftarrow \text { even } \wedge \bigvee_{-2 r_{2} / d<k<-2 r_{1} / d}\left(\bigcirc^{k} P_{2}^{\nu\left(\boldsymbol{\tau}_{2}\right)} \wedge \bigcirc^{k} \text { odd } \wedge \bigwedge_{k \leq j<0} \bigcirc^{j} P_{1}^{\nu\left(\boldsymbol{\tau}_{1}\right)}\right),\right. \\
\square\left(P^{\nu(\boldsymbol{\tau})} \leftarrow \text { odd } \wedge \bigvee_{-2 r_{2} / d \leq k \leq-2 r_{1} / d}\left(\bigcirc^{k} P_{2}^{\nu\left(\boldsymbol{\tau}_{2}\right)} \wedge \bigcirc^{k} \text { even } \wedge \bigwedge_{k<j \leq 0} \bigcirc^{j} P_{1}^{\nu\left(\boldsymbol{\tau}_{1}\right)}\right),\right. \\
\square\left(P^{\nu(\boldsymbol{\tau})} \leftarrow \text { odd } \wedge \bigvee_{-2 r_{2} / d \leq k \leq-2 r_{1} / d}\left(\bigcirc^{k} P_{2}^{\nu\left(\boldsymbol{\tau}_{2}\right)} \wedge \bigcirc^{k} \text { odd } \wedge \bigwedge_{k \leq j \leq 0} \bigcirc^{j} P_{1}^{\nu\left(\boldsymbol{\tau}_{1}\right)}\right) ;\right.
\end{gathered}
$$

- for every rule $P(\boldsymbol{\tau}) \leftarrow P_{1}\left(\boldsymbol{\tau}_{1}\right) \mathcal{S}_{\varrho} P_{2}\left(\boldsymbol{\tau}_{2}\right)$ in $\Pi$ with $\varrho=\left(r_{1}, \infty\right)$,

$$
\begin{array}{r}
\square\left(P ^ { \nu ( \boldsymbol { \tau } ) } \leftarrow \operatorname { e v e n } \wedge \bigwedge _ { - 2 r _ { 1 } / d \leq j < 0 } \bigcirc ^ { j } P _ { 1 } ^ { \nu ( \boldsymbol { \tau } _ { 1 } ) } \wedge \bigcirc ^ { - 2 r _ { 1 } / d } \left(P_{1}^{\nu\left(\boldsymbol{\tau}_{1}\right)} \mathcal{S}\left(\text { even } \wedge P_{2}^{\nu\left(\boldsymbol{\tau}_{2}\right)}\right) \vee\right.\right. \\
\left.\left.P_{1}^{\nu\left(\boldsymbol{\tau}_{1}\right)} \mathcal{S}\left(\text { odd } \wedge P_{1}^{\nu\left(\boldsymbol{\tau}_{1}\right)} \wedge P_{2}^{\nu\left(\boldsymbol{\tau}_{2}\right)}\right)\right)\right), \\
\square\left(P ^ { \nu ( \boldsymbol { \tau } ) } \leftarrow \operatorname { o d d } \wedge \bigwedge _ { - 2 r _ { 1 } / d \leq j \leq 0 } \bigcirc ^ { j } P _ { 1 } ^ { \nu ( \boldsymbol { \tau } _ { 1 } ) } \wedge \bigcirc ^ { - 2 r _ { 1 } / d } \left(P_{2}^{\nu\left(\boldsymbol{\tau}_{2}\right)} \vee P_{1}^{\nu\left(\boldsymbol{\tau}_{1}\right)} \mathcal{S}\left(\operatorname{even} \wedge P_{2}^{\nu\left(\boldsymbol{\tau}_{2}\right)}\right) \vee\right.\right. \\
\left.\left.P_{1}^{\nu\left(\boldsymbol{\tau}_{1}\right)} \mathcal{S}\left(\text { odd } \wedge P_{1}^{\nu\left(\boldsymbol{\tau}_{1}\right)} \wedge P_{2}^{\nu\left(\boldsymbol{\tau}_{2}\right)}\right)\right)\right)
\end{array}
$$

(recall that $P \mathcal{S} Q$ holds at $i$ iff there exists $k<i$, such that $Q$ holds at $k$ and $P$ holds at all $j$ with $k<j<i$ );

- similar clauses for the rules of the form $P(\boldsymbol{\tau}) \leftarrow P_{1}\left(\boldsymbol{\tau}_{1}\right) \mathcal{U}_{\varrho} P_{2}\left(\boldsymbol{\tau}_{2}\right)$ (here we need the 'until' operator $\mathcal{U}), P(\boldsymbol{\tau}) \leftarrow \boxminus_{\varrho} P_{1}\left(\boldsymbol{\tau}_{1}\right)$ and $P(\boldsymbol{\tau}) \leftarrow \boxplus_{\varrho} P_{1}\left(\boldsymbol{\tau}_{1}\right)$ in $\Pi$; 
- for every fact $P(\boldsymbol{c}) @_{\iota}$ in $\mathcal{D}$, we need the clauses:

$$
\begin{array}{ll}
\bigcirc^{2 r / d} P^{c}, & \text { if } \iota=[r, r], \\
\bigwedge_{2 r_{1} / d<i<2 r_{2} / d} \bigcirc^{i} P^{c}, & \text { if } \iota=\left(r_{1}, r_{2}\right) \text { and } r_{1}, r_{2} \in \mathbb{Q}_{2}, \\
\bigcirc^{2 r_{1} / d} \square_{F} P^{c}, & \text { if } \iota=\left(r_{1}, r_{2}\right), r_{1} \in \mathbb{Q}_{2} \text { and } r_{2}=\infty, \\
\bigcirc^{2 r_{2} / d} \square_{P} P^{c}, & \text { if } \iota=\left(r_{1}, r_{2}\right), r_{1}=-\infty \text { and } r_{2} \in \mathbb{Q}_{2}, \\
\square_{F} \square_{P} P^{c}, & \text { if } \iota=\left(r_{1}, r_{2}\right), r_{1}=-\infty \text { and } r_{2}=\infty .
\end{array}
$$

Lemma 7. $(\Pi, D)$ is consistent iff $\varphi_{\Pi, \mathcal{D}}$ is satisfiable.

Proof. $(\Rightarrow)$ If $\mathfrak{C}_{\Pi, \mathcal{D}}$ is a model of $(\Pi, \mathcal{D})$, we define an $L T L$-interpretation $\mathfrak{M}$ by taking

- $\mathfrak{M}, i \models P^{c}$ iff $\mathfrak{C}_{\Pi, \mathcal{D}}, t \models P(\boldsymbol{c})$, for all $t \in \sigma_{i}$ and $i \in \mathbb{Z}$, all tuples of individual constants $\boldsymbol{c}$, and predicates $P$;

- $\mathfrak{M}, i \models$ even, for even $i \in \mathbb{Z}$;

- $\mathfrak{M}, i \models$ odd, for odd $i \in \mathbb{Z}$.

It is routine to check that $\mathfrak{M}, 0 \models \varphi_{\Pi, \mathcal{D}}$, taking into account that $\mathfrak{C}_{\Pi, \mathcal{D}}, t \models P_{1}\left(\boldsymbol{c}_{1}\right) \mathcal{S}_{\varrho} P_{2}\left(\boldsymbol{c}_{2}\right)$ for some (= all) $t \in \sigma_{i}$ iff the following conditions hold:

Case $\varrho=[r, r]: \mathfrak{C}_{\Pi, \mathcal{D}}, t^{\prime}=P_{2}\left(\boldsymbol{c}_{2}\right)$, for some $t^{\prime} \in \sigma_{i-2 r / d}$, and $\mathfrak{C}_{\Pi, \mathcal{D}}, s=P_{1}\left(\boldsymbol{c}_{1}\right)$ for all $s \in \sigma_{j}$ such that

$$
\begin{array}{ll}
i-2 r / d<j<i, & \text { if } i \text { is even, } \\
i-2 r / d \leq j \leq i, & \text { if } i \text { is odd }
\end{array}
$$

Case $\varrho=\left(r_{1}, r_{2}\right)$ : there exists $\sigma_{k}$ with $\mathfrak{C}_{\Pi, \mathcal{D}}, t^{\prime} \models P_{2}\left(\boldsymbol{c}_{2}\right)$, for some $t^{\prime} \in \sigma_{k}$, and $\mathfrak{C}_{\Pi, \mathcal{D}}, s \models P_{1}\left(\boldsymbol{c}_{1}\right)$ for all $s \in \sigma_{j}$ such that

$$
\begin{aligned}
& i-2 r_{2} / d<k<i-2 r_{1} / d, \quad k<j<i, \quad \text { if } i \text { is even and } k \text { is even, } \\
& i-2 r_{2} / d<k<i-2 r_{1} / d, \quad k \leq j<i, \quad \text { if } i \text { is even and } k \text { is odd, } \\
& i-2 r_{2} / d \leq k \leq i-2 r_{1} / d, \quad k<j \leq i, \quad \text { if } i \text { is odd and } k \text { is even, } \\
& i-2 r_{2} / d \leq k \leq i-2 r_{1} / d, \quad k \leq j \leq i, \quad \text { if } i \text { is odd and } k \text { is odd; }
\end{aligned}
$$

and similarly for the other temporal operators in $\varphi_{\Pi, \mathcal{D}}$.

$(\Leftarrow)$ Suppose now $\varphi_{\Pi, \mathcal{D}}$ is satisfiable. Take the canonical model $\mathfrak{M}$ of $\varphi_{\Pi, \mathcal{D}}$ with $\mathfrak{M}, 0=\varphi_{\Pi, \mathcal{D}}$; see the work by Artale, Kontchakov, Ryzhikov, and Zakharyaschev (2013) for details. Using the observations above, it is not hard to check that $\mathfrak{M}, i \models P^{c}$ iff $\mathfrak{C}_{\Pi, \mathcal{D}}, t \models P(\boldsymbol{c})$, for all $t \in \sigma_{i}$ and $i \in \mathbb{Z}$, all tuples of individual constants $c$ and predicates $P$. Details are left to the reader.

We are now in a position to prove our first complexity result:

Theorem 8. Consistency checking for datalogMTL programs is EXPSPACE-complete. The lower bound holds even for propositional datalogMTL. 
Proof. We first show the upper bound. By the two lemmas above, a datalogMTL program $\Pi$ is consistent with a data instance $\mathcal{D}$ iff the $L T L$ formula $\varphi_{\Pi, \mathcal{D}}$ is satisfiable. Thus, a consistency checking EXPSPACE algorithm can first construct $\varphi_{\Pi, \mathcal{D}}$, which requires exponential time in the size of $\Pi$ and $\mathcal{D}$. Indeed, the greatest common divisor of the set num $(\Pi, \mathcal{D})$ can be computed in polynomial time. The $L T L$ formula $\varphi_{\Pi, \mathcal{D}}$ contains exponentially many clauses (as there are exponentially many assignments $\nu$ ) of at most exponential size (as they contain $2 t / d$ conjuncts or disjuncts, where $t$ is a number from $\Pi$ or $\mathcal{D}$ ). After that we can run a standard PSPACE satisfiability checking algorithm for LTL; see, e.g., the work by Sistla and Clarke (1985).

We establish the matching lower bound by reduction of the non-halting problem for deterministic Turing machines with an exponential tape. Let $M$ a deterministic Turing machine that requires $2^{f(m)}$ cells of the tape given an input of length $m$, for some polynomial $f$. Let $n=f(m)$. Without loss of generality, we can assume that $M$ never runs outside the first $2^{n}$ cells. Suppose $M=\left(Q, \Gamma, \#, \Sigma, \delta, q_{0}, q_{h}\right)$, where $Q$ is a finite set of states, $\Gamma$ a tape alphabet, $\# \in \Gamma$ the blank symbol, $\Sigma \subseteq \Gamma$ a set of input symbols, $\delta:\left(Q \backslash\left\{q_{h}\right\}\right) \times \Gamma \rightarrow Q \times \Gamma \times\{L, R\}$ a transition function, and $q_{0}, q_{h} \in Q$ are the initial and halting states, respectively. Let $\vec{a}=a_{1} \ldots a_{m}$ be an input for $M$. We construct a propositional datalogMTL program $\Pi$ and a data instance $\mathcal{D}$ such that they are not consistent iff $M$ accepts $\vec{a}$. In our encoding, we employ the following propositional variables, where $a \in \Gamma, q \in Q$ :

- $H_{q, a}$ indicating that a cell is read by the head, the current state of the machine is $q$, and the cell contains $a$;

- $N_{a}$ indicating that a cell is not read by the head and contains $a$,

- first and last marking the first and last cells of a configuration, respectively.

The program $\Pi$ consists of the following rules, for $a, a^{\prime}, a^{\prime \prime} \in \Gamma, q, q^{\prime} \in Q$ :

$$
\begin{array}{ll}
\boxplus_{2^{n}+1} H_{q^{\prime}, a^{\prime \prime}} \leftarrow H_{q, a} \wedge \boxplus_{1} N_{a^{\prime \prime}}, \quad \boxplus_{2^{n}} N_{a^{\prime}} \leftarrow H_{q, a}, & \text { if } \delta(q, a)=\left(q^{\prime}, a^{\prime}, R\right), \\
\boxplus_{2^{n}-1} H_{q^{\prime}, a^{\prime \prime}} \leftarrow H_{q, a} \wedge \boxminus_{1} N_{a^{\prime \prime}}, \boxplus_{2^{n}} N_{a^{\prime}} \leftarrow H_{q, a}, & \text { if } \delta(q, a)=\left(q^{\prime}, a^{\prime}, L\right), \\
\boxplus_{2^{n}} N_{a} \leftarrow \boxminus_{1} N_{a^{\prime}} \wedge N_{a} \wedge \boxplus_{1} N_{a^{\prime \prime}}, & \\
\boxplus_{2^{n}} N_{a} \leftarrow \boxminus_{1} H_{q, a^{\prime}} \wedge N_{a} \wedge \boxplus_{1} N_{a^{\prime \prime}}, & \text { if } \delta\left(q, a^{\prime}\right) \neq(r, b, R) \text { for all } r, b, \\
\boxplus_{2^{n}} N_{a} \leftarrow \boxminus_{1} N_{a^{\prime}} \wedge N_{a} \wedge \boxplus_{1} H_{q, a^{\prime \prime}}, & \text { if } \delta\left(q, a^{\prime \prime}\right) \neq(r, b, L) \text { for all } r, b, \\
\boxplus_{2^{n}} N_{a} \leftarrow N_{a} \wedge \text { first } \wedge \boxplus_{1} N_{a^{\prime}}, & \\
\boxplus_{2^{n}} N_{a} \leftarrow N_{a} \wedge \text { first } \wedge \boxplus_{1} H_{q, a^{\prime}}, & \text { if } \delta\left(q, a^{\prime}\right) \neq(r, b, L) \text { for all } r, b, \\
\boxplus_{2^{n}} N_{a} \leftarrow \boxminus_{1} N_{a^{\prime}} \wedge N_{a} \wedge \text { last, } & \\
\boxplus_{2^{n}} N_{a} \leftarrow \boxminus_{1} N_{q, a^{\prime}} \wedge N_{a} \wedge \text { last, } & \\
\boxplus_{2^{n}} \text { first } \leftarrow \text { first, } & \\
\boxplus_{2^{n}} \text { last } \leftarrow \text { last, } & \\
\perp \leftarrow H_{q_{h}, a}, & \\
\boxplus_{1} N_{\#} \leftarrow N_{\#} \wedge \bigoplus_{(0, \infty)} N_{\#}^{<,} &
\end{array}
$$

where $\boxplus_{r}$ is an abbreviation for $\boxplus_{[r, r]}$ and similarly for $\boxminus_{r}$. Let $\mathcal{D}$ contain the following facts:

$$
\begin{aligned}
N_{a_{i}} @[i, i], \text { for } 1<i \leq m, N_{\#} @[m+1, m+1], \quad N_{\#}^{<} @\left[2^{n}, 2^{n}\right], \\
H_{q_{0}, a_{1}} @[1,1], \text { first } @[1,1], \quad \text { last } @\left[2^{n}, 2^{n}\right] .
\end{aligned}
$$


The program represents the computation of $M$ on $\vec{a}$ as a sequence of configurations. The initial one is spread over the time instants $1, \ldots, 2^{n}$, from which the first $m$ instants represent $\vec{a}$ and the remaining ones are \#. The second configuration uses the next $2^{n}$ instants (i.e., $2^{n}+1, \ldots, 2^{n}+2^{n}$ ), etc. It is routine to check that $M$ halts on $\vec{a}$ iff $\Pi$ and $\mathcal{D}$ are inconsistent.

Note that datalogMTL allows punctual intervals of the form $[r, r]$ as ranges of temporal operators, and that full propositional MTL with such intervals is undecidable (Alur \& Henzinger, 1993).

Now we turn to the data complexity of datalogMTL and show the following result:

Theorem 9. Consistency checking and answering propositional datalogMTL queries is $\mathrm{P}$-hard for data complexity (under LOGSPACE reductions).

Proof. We establish this lower bound by reduction of the monotone circuit value problem, which is known to be P-complete (Arora \& Barak, 2009). Let $C$ be a monotone circuit with input gates having fan-in 1 and all other gates fan-in 2 . We assume that the gates are enumerated by consecutive positive integers, so that if there is an edge from $n$ to $m$ then $n<m$. Let $N=2^{k}$, for some $k \in \mathbb{N}$, be the minimal number that is greater than or equal to the maximal gate number. We encode the computation of $\boldsymbol{C}$ on an input $\boldsymbol{\alpha}$ by a data instance $\mathcal{D}_{\boldsymbol{C}}$ with the following punctual facts, where $[n]$ stands for $[n, n]$ :

$-V[2 n+n / N]$, if $n$ is an input gate and $\boldsymbol{\alpha}(n)=V \in\{T, F\}$;

$-D[2 n+n / N]$, if $n$ is an OR gate;

- $C[2 n+n / N]$, if $n$ is an AND gate;

- $I_{0}[2 n+m / N], I_{1}[2 n+k / N]$, if $n$ is a gate with input gates $m$ and $k$.

Let $\Pi_{C}$ be a datalogMTL program with the rules

$$
\begin{array}{ll}
T \leftarrow \vartheta_{[2,2]} T, & F \leftarrow \vartheta_{[2,2]} F, \\
T \leftarrow \vartheta_{[0,1]}\left(I_{0} \wedge T\right) \wedge D, & F \leftarrow \vartheta_{[0,1]}\left(I_{0} \wedge F\right) \wedge C, \\
T \leftarrow \vartheta_{[0,1]}\left(I_{1} \wedge T\right) \wedge D, & F \leftarrow \vartheta_{[0,1]}\left(I_{1} \wedge F\right) \wedge C, \\
F \leftarrow \vartheta_{[0,1]}\left(I_{0} \wedge F\right) \wedge \vartheta_{[0,1]}\left(I_{1} \wedge F\right) \wedge D, & T \leftarrow \vartheta_{[0,1]}\left(I_{0} \wedge T\right) \wedge \vartheta_{[0,1]}\left(I_{1} \wedge T\right) \wedge C .
\end{array}
$$

Suppose $n$ is the output gate. Then it is straightforward to check that the value of $\mathbf{C}$ on $\boldsymbol{\alpha}$ is $T$ iff $(\Pi, \mathcal{D}) \models T[2 n+n / N]$. This immediately implies the required hardness for the query answering problem. An example of a circuit $\boldsymbol{C}$ with an assignment $\boldsymbol{\alpha}$, and an initial part of the canonical model of $\left(\Pi_{C}, \mathcal{D}_{C}\right)$ are shown below, with the black symbols above the timestamps indicating what is given in $\mathcal{D}_{C}$ and the grey ones what is implied by $\Pi_{C}$ :
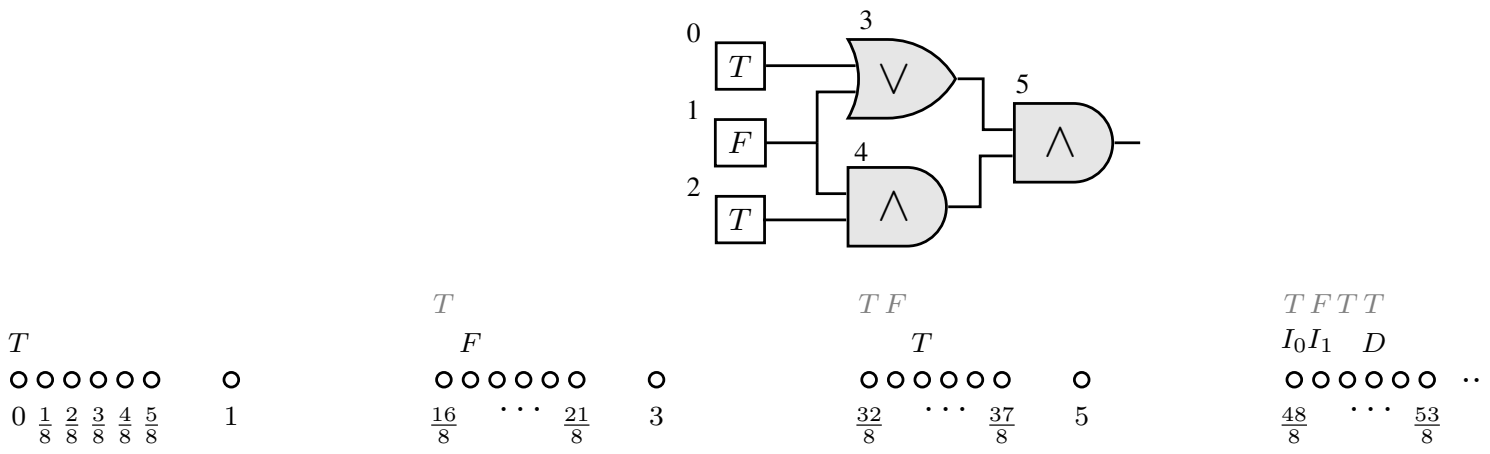
To show P-hardness of the consistency problem, it suffices to add the fact $P[2 n+n / N]$ to $\mathcal{D}_{\boldsymbol{C}}$, for a fresh $P$, and the axiom $\perp \leftarrow P \wedge T$ to $\Pi_{C}$.

The exact data complexity of answering propositional datalogMTL queries remains open. It is worth noting that answering ontology-mediated queries with propositional LTL ontologies is $\mathrm{NC}^{1}$-complete for data complexity (Artale et al., 2015), while answering propositional datalog queries with the Halpern-Shoham operators is P-complete for data complexity (Kontchakov, Pandolfo, Pulina, Ryzhikov, \& Zakharyaschev, 2016).

The diamond operators $\vartheta_{\varrho}$ and $\vartheta_{\varrho}$ are disallowed in the head of datalogMTL rules. Denote by datalogMTL ${ }^{\diamond}$ the extension of datalogMTL that allows both box and diamond operators in the head of rules. We show now that this language has much more expressive power and can encode 2-counter Minsky machines, which gives the following theorem; cf. the work by Madnani, Krishna, and Pandya (2013):

Theorem 10. Consistency checking for propositional datalog $\mathrm{MTL}^{\diamond}$ programs is undecidable.

Proof. We use some ideas of Madnani et al. (2013), where a non-Horn fragment of MTL was shown to be undecidable. The proof is by reduction of the undecidable non-halting problem for Minksy machines: given a 2-counter Minsky machine, decide whether it does not halt starting from 0 in both counters.

Suppose we are given a Minsky machine with counters $C_{1}$ and $C_{2}$ that has $n-1$ instructions of the form

$$
\begin{aligned}
& i \text { : Increment }\left(C_{k}\right) \text {, goto } j, \\
& i \text { : Decrement }\left(C_{k}\right) \text {, goto } j, \\
& i \text { : If } C_{k}=0 \text { then } j_{1} \text { else } j_{2},
\end{aligned}
$$

where $i, j, j_{1}$ and $j_{2}$ are instruction indexes, $k=1,2$, and the $n$-th instruction is

$$
n \text { : Halt. }
$$

We encode successive configurations of the machine using the sequence $[0,4),[4,8),[8,12), \ldots$ of time intervals. The current instruction index is represented by a propositional variable $P_{i}$, for $1 \leq i \leq n$, that holds at the first point, say $4 m$, of the interval $[4 m, 4 m+4)$. The current value, say $k_{1}$, of the counter $C_{1}$ is encoded by exactly $k_{1}$ moments of time in the interval $(4 m+1,4 m+2)$ where the propositional variable $C$ holds true. Similarly, the value $k_{2}$ of $C_{2}$ is encoded by exactly $k_{2}$ moments in the interval $(4 m+3,4 m+4)$ where the propositional variable $C$ holds true.

The initial configuration is encoded by the following data instance $\mathcal{D}$, where the variable $Z$ indicates that both counters are 0 :

$$
P_{1} @[0,0], \quad Z @(1,2), \quad Z @(3,4) .
$$

For every $i(1 \leq i \leq n)$ we require the rules

$$
\boxplus_{[0,1]} Z \leftarrow P_{i}, \quad \boxplus_{[2,3]} Z \leftarrow P_{i}, \quad \perp \leftarrow Z \wedge C, \quad \perp \leftarrow Z \wedge N
$$

saying, in particular, that $C$ cannot hold true outside the intended intervals (here $N$ is an auxiliary variable). To simplify notation, we use the following abbreviations: (1) $=\boxplus_{[1,1]}$, (2) $=\boxplus_{[3,3]}$, and 
$\bigcirc=\boxplus_{[4,4]}$. The machine instructions are encoded as follows (the instructions for $C_{2}$ are obtained by replacing (1) with (2):

$$
\begin{array}{ll}
\bigcirc P_{j_{1}} \leftarrow P_{i} \wedge \mathbb{1}_{(0,1)} Z, & \\
\bigcirc P_{j_{2}} \leftarrow P_{i} \wedge \text { (1) } \uplus_{(0,1)} C, & i \text { : if } C_{1}=0 \text { then } j_{1} \\
\text { (1) } \boxplus_{(0,1)} \mathrm{CP} \leftarrow P_{i}, \quad \text { (2) } \boxplus_{(0,1)} \mathrm{CP} \leftarrow P_{i}, & \text { else } j_{2} \\
\bigcirc P_{j} \leftarrow P_{i}, \quad(1) \boxplus_{(0,1)} \mathrm{IC} \leftarrow P_{i}, \quad \text { (2) } \boxplus_{(0,1)} \mathrm{CP} \leftarrow P_{i}, & i: \operatorname{Inc}\left(C_{1}\right), \text { goto } j \\
O P_{j} \leftarrow P_{i}, \quad(1) \boxplus_{(0,1)} \mathrm{DC} \leftarrow P_{i}, \quad \text { (2) } \boxplus_{(0,1)} \mathrm{CP} \leftarrow P_{i}, & i: \operatorname{Dec}\left(C_{1}\right), \text { goto } j .
\end{array}
$$

Here the variable $\mathrm{CP}$ means copying of the counter value, DC means decrementing it by 1 , and IC incrementing it by 1 . To achieve this, we require the following rules:

$$
\begin{aligned}
& \bigcirc C \leftarrow \mathrm{CP} \wedge C, \quad \bigcirc Z \leftarrow \mathrm{CP} \wedge Z, \\
& \bigcirc C \leftarrow \mathrm{DC} \wedge C \wedge \oplus_{(0,1)} C, \\
& \bigcirc Z \leftarrow \mathrm{DC} \wedge Z \wedge \ominus_{(0,1)} C, \quad \bigcirc \boxplus_{[0,1]} Z \leftarrow \mathrm{DC} \wedge C \wedge \boxplus_{(0,1)} Z, \\
& \ominus_{(0,1)} N \leftarrow \boxplus_{(0,1)} \mathrm{IC} \wedge \boxplus_{(0,1)} Z, \quad \vartheta_{(0,1)} N \leftarrow C \wedge \mathrm{IC} \wedge \boxplus_{(0,1)} Z, \\
& \bigcirc C \leftarrow \mathrm{IC} \wedge C, \quad \bigcirc C \leftarrow \mathrm{IC} \wedge N, \\
& \bigcirc Z \leftarrow \mathrm{IC} \wedge Z \wedge \oplus_{(0,1)} N, \quad \bigcirc \boxplus_{(0,1)} Z \leftarrow \mathrm{IC} \wedge N \wedge \boxplus_{(0,1)} Z,
\end{aligned}
$$

We explain the intuition behind the most complex rules (8)-(10) that are used to model the increment of the counters. The rules (8) mark a new time-point with the variable $N$ in a block located after the last $C$-time-point in this block (or, according the first axiom, $N$ is placed anywhere in the block if the current value of a counter is 0). The rules (9) insert $C$ in the next block, where in the current block we have either $C$ or $N$. The rules (10) transfer $Z$ from the current block to the next one excluding the time-point where $N$ holds. Finally, we add the rule

$$
\perp \leftarrow P_{n}, \quad \text { n: Halt. }
$$

It is not hard to check that the program and data instance above are consistent iff the given 2-counter Minsky machine does not halt.

The diamond operators in the head of rules can encode disjunction and thereby ruin 'Horness'. Thus, the temporalised description logic $\mathcal{E} \mathcal{L}$ with such rules is undecidable (Lutz, Wolter, \& Zakharyaschev, 2008); cf. also the work by Gutiérrez-Basulto et al. (2016a). The addition of diamonds in the heads to the Horn fragment of the propositional Halpern-Shoham logic $\mathcal{H S}$ can make a P-complete logic undecidable (Bresolin et al., 2017). A distinctive feature of these formalisms is their two-dimensionality (Gabbay, Kurucz, Wolter, \& Zakharyaschev, 2003), while propositional datalogMTL is one-dimensional. Diamonds in the head of rules also ruin FO-rewritability of answering ontology-mediated queries with temporalised DL-Lite ontologies by increasing their data complexity to CONP (Artale et al., 2013). The same construction actually shows that nonrecursive datalogMTL with binary predicates and diamonds in the heads is CONP-hard.

\section{Nonrecursive DatalogMTL}

As none of the datalogMTL programs required in our use cases is recursive, we now consider the class datalog $\log _{n r} M T L$ of nonrecursive datalogMTL programs. We first show that consistency 
checking (and so query answering) for datalog ${ }_{n r} M T L$ programs is PSPACE-complete for combined complexity. Then we regard a given datalog ${ }_{n r} M T L$ program as fixed and reduce these problems to evaluating a (data-independent) $\mathrm{FO}(<)$-formula over any given data, thereby establishing that datalog $\log _{n r} M T L$ is in $\mathrm{AC}^{0}$ for data complexity.

More precisely, for a program $\Pi$, let $\lessdot$ be the dependence relation on the predicate symbols in $\Pi$ : we have $P \lessdot Q$ iff $\Pi$ contains a clause with $P$ in the head and $Q$ in the body. $\Pi$ is called nonrecursive if $P \lessdot^{+} P$ does not hold for any predicate symbol $P$ in $\Pi$, where $\lessdot^{+}$is the transitive closure of $\lessdot$. We denote by $\operatorname{depth}_{\Pi}(P)$ the maximal number $l$ such that $P_{0} \lessdot P_{1} \lessdot \cdots \lessdot P_{l}=P$. (Note that $\operatorname{depth}_{\Pi}(P)=0$ iff either $P$ does not occur in $\Pi$ or $P$ occurs only in the body of some rules.) The maximal $\operatorname{depth}_{\Pi}(P)$ over all predicates $P$ is denoted by depth $(\Pi)$. It should be clear that, for any nonrecursive $\Pi$ and any data instance $\mathcal{D}$, there exists some $n \in \mathbb{N}$ such that $\mathrm{cl}^{n+1}(\mathcal{D})=$ $\mathrm{cl}^{n}(\mathcal{D})=\mathfrak{C}_{\Pi, \mathcal{D}}$. Therefore, $\mathfrak{C}_{\Pi, \mathcal{D}}$ is finite.

Denote by $\min \mathcal{D}$ and $\max \mathcal{D}$ the minimal and, respectively, maximal finite numbers that occur in the intervals from $\mathcal{D}$. Let $K$ be the largest number occurring in $\Pi$. We then set

$$
M_{l}=\min \mathcal{D}-K \times \operatorname{depth}(\Pi) \quad \text { and } \quad M_{r}=\max \mathcal{D}+K \times \operatorname{depth}(\Pi) .
$$

Let $d=\operatorname{gcd}(\operatorname{num}(\Pi, \mathcal{D}))$. The next lemma will be required for our PSPACE algorithm checking consistency of datalog $\log _{n r} M T L$ programs.

Lemma 11. Let $\Pi$ be a datalog ${ }_{n r} M T L$ program. Then every interval $\iota$ such that $P(\boldsymbol{c}) @ \iota \in \mathfrak{C}_{\Pi, \mathcal{D}}$ or $\perp(\boldsymbol{c}) @_{\iota} \in \mathfrak{C}_{\Pi, \mathcal{D}}$ takes one of the following forms: $(-\infty, \infty),\langle d k, \infty),(-\infty, d k\rangle,\left\langle d k, d k^{\prime}\right\rangle$, where $k, k^{\prime} \in \mathbb{Z}$ and $M_{l} \leq d k \leq d k^{\prime} \leq M_{r}$.

Proof. That every interval in $\mathfrak{C}_{\Pi, \mathcal{D}}$ is of the form $(-\infty, \infty),\langle d k, \infty),(-\infty, d k\rangle,\left\langle d k, d k^{\prime}\right\rangle$, where $k, k^{\prime} \in \mathbb{Z}$, was observed in the proof of Lemma 6 . Thus, we only need to establish the bounds on $d k$ and $d k^{\prime}$. For each $P$, let hi $(P)$ and $\mathrm{lo}(P)$ be the maximal and, respectively, minimal number $d k \in \mathbb{Q}$ such that $P(\boldsymbol{c}) @ \iota \in \mathfrak{C}_{\Pi, \mathcal{D}}$ and $d k$ is an end-point of $\iota$. Note that $h i(P)$ and $\operatorname{lo}(P)$ can be undefined. We are going to show that hi $(P)$ is either undefined or hi $(P) \leq \max \mathcal{D}+\operatorname{depth}_{\Pi}(P) K$. (That $\operatorname{lo}(P)$ is either undefined or $\operatorname{lo}(P) \geq \min \mathcal{D}-\operatorname{depth}_{\Pi}(P) K$ is left to the reader.) Clearly, this fact implies the required bounds on $d k$ and $d k^{\prime}$.

The proof is by induction on the construction of $\mathfrak{C}_{\Pi, \mathcal{D}}$. Let hi ${ }^{n}(P)$ be the maximal $d k \in \mathbb{Q}_{2}$ such that $P(\boldsymbol{c}) @ \iota \in \mathrm{cl}^{n}(\mathcal{D})$ and $d k$ is an end-point of $\iota$. We show by induction on $n$ that either $\mathrm{hi}^{n}(P)$ is undefined or hi ${ }^{n}(P) \leq \max \mathcal{D}+K \operatorname{depth}_{\Pi}(P)$.

For the basis of induction, if $\mathrm{hi}^{0}(P)$ is defined and $P(\boldsymbol{c}) @ \iota \in \mathrm{cl}^{0}(\mathcal{D})$ is an atom mentioning $\mathrm{hi}^{0}(P)$, then $P(\boldsymbol{c}) @ \iota \in \mathcal{D}$ and $\mathrm{hi}^{0}(P) \leq \max \mathcal{D}$. Assume next that $n=n^{\prime}+1$. Suppose $\mathrm{hi}^{n}(P)$ is defined and let $P(\boldsymbol{c}) @_{\iota} \in \mathrm{cl}^{n}(\mathcal{D})$ be an atom mentioning hi ${ }^{n}(P)$. If $P(\boldsymbol{c}) @ \iota \in \mathrm{cl}^{n^{\prime}}(\mathcal{D})$, we are done by the induction hypothesis. Otherwise, we consider how $P(\boldsymbol{c}) @ \iota$ was obtained. Suppose it was obtained by (coal) with $\iota=\bigcup_{i \in I} \iota_{i}$. By the induction hypothesis, hi $^{n^{\prime}}(P) \leq \max \mathcal{D}+K \operatorname{depth}_{\Pi}(P)$, and so every number mentioned in $\left\{\iota_{i} \mid i \in I\right\}$ does not exceed max $\mathcal{D}+K \operatorname{depth}_{\Pi}(P)$. Thus, we have $\mathrm{hi}^{n}(P) \leq \max \mathcal{D}+K \operatorname{depth}_{\Pi}(P)$. Now suppose that $P(\boldsymbol{c}) @ \iota$ was obtained by (horn) from $P_{i}\left(\boldsymbol{c}_{i}\right) @ \iota_{i}, i \in I$. Observe that $\operatorname{depth}_{\Pi}\left(P_{i}\right)<\operatorname{depth}_{\Pi}(P)$ and, by the induction hypothesis, $\operatorname{hi}^{n^{\prime}}\left(P_{i}\right) \leq \max \mathcal{D}+K \operatorname{depth}_{\Pi}\left(P_{i}\right)$. Since $\iota=\bigcap_{i \in I} \iota_{i}$, the maximal number mentioned in $\iota$ cannot exceed $\max \mathcal{D}+K \operatorname{depth}_{\Pi}(P)$. Thus, hi $^{n}(P) \leq \max \mathcal{D}+K \operatorname{depth}_{\Pi}(P)$. Consider now the case when $P(\boldsymbol{c}) @ \iota$ was obtained by applying $\left(\mathcal{S}_{\varrho}\right)$ to $P_{i}\left(\boldsymbol{c}_{i}\right) @ \iota_{i}, i \in\{1,2\}$. By the induction hypothesis, the largest number mentioned in $\iota_{i}$ does not exceed $\max \mathcal{D}+K \operatorname{depth}_{\Pi}\left(P_{i}\right)$. On the other hand, 
$\operatorname{depth}_{\Pi}\left(P_{i}\right)<\operatorname{depth}_{\Pi}(P)$ and the maximal number in $\iota$ cannot be larger that the maximal number in $\left\{\iota_{i} \mid i \in\{1,2\}\right\}$ plus $K$. Thus, the maximal number in $\iota$ does not exceed

$$
\max \mathcal{D}+K \operatorname{depth}_{\Pi}\left(P_{i}\right)+K \leq \max \mathcal{D}+K \operatorname{depth}_{\Pi}(P),
$$

and so $\mathrm{hi}^{n}(P) \leq \max \mathcal{D}+K \operatorname{depth}_{\Pi}(P)$. The remaining temporal rules are similar and left to the reader.

Suppose we are given a datalog ${ }_{n r} M T L$ program $\Pi$ and a data instance $\mathcal{D}$. If $\Pi$ and $\mathcal{D}$ are inconsistent then, by Lemmas 5 and 11, we have $\perp @ \iota \in \mathfrak{C}_{\Pi, \mathcal{D}}$, for some $\iota$ of the form $(-\infty, \infty)$, $\langle d k, \infty),(-\infty, d k\rangle,\left\langle d k, d k^{\prime}\right\rangle$, where $k, k^{\prime} \in \mathbb{Z}$ and $M_{l} \leq d k \leq d k^{\prime} \leq M_{r}$. Thus, there is a derivation of $\perp @ \iota$ from $\Pi$ and $\mathcal{D}$, that is, a tree whose root is $\perp @ \iota$, whose leaves are some atoms from $\mathcal{D}$, and whose every non-leaf vertex results from applying one of the rules (coal), (horn), $\left(\mathcal{S}_{\varrho}\right)$, $\left(\boxplus_{\varrho}\right),\left(\mathcal{U}_{\varrho}\right),\left(\boxminus_{\varrho}\right)$ to the immediate predecessors of this vertex.

Lemma 12. If $\perp @ \iota \in \mathfrak{C}_{\Pi, \mathcal{D}}$ then there is a derivation of $\perp @_{\iota}$ from $\Pi$ and $\mathcal{D}$ such that

(i) the length of any branch in the derivation does not exceed 2|П|;

(ii) for some polynomial p, every non-leaf vertex, corresponding to the application of (coal) in the derivation, has at most $2^{p(|\Pi|,|\mathcal{D}|)}$ immediate predecessors.

Proof. To show $(i)$, it suffices to recall that $\Pi$ is non-recursive (and so none of the rules in $\Pi$ can be applied twice in the same branch of the derivation) and observe that we can always replace multiple successive applications of the rule (coal) with a single application.

(ii) follows from Lemma 11.

Theorem 13. Consistency checking for datalog ${ }_{n r}$ MTL programs is PSPACE-complete for combined complexity. The lower bound holds even for propositional datalog ${ }_{n r} M T L$.

Proof. The upper bound is established by a standard algorithm (Ladner, 1977; Tobies, 2001) using Lemma 12 and Savitch's theorem according to which NPSPACE = PSPACE. In essence, the NPSPACE algorithm guesses branches of the derivation one by one and keeps only last two branches in memory. By Lemma $12(i)$, each branch contains $\leq 2|\Pi|$ atoms of the form $P(\boldsymbol{c}) @ \iota$, where $\iota$ is as in Lemma 11, and so is stored in polynomial space. In addition, we store the axioms in $\Pi$ that created these atoms, or (coal) if the atom was obtained by coalescing. In the latter case, we also need to guess a number $k$ indicating how many distinct intervals are coalesced to obtain $\iota$. By Lemma $12(i i), k \leq 2^{p(|\Pi|,|\mathcal{D}|)}$, and so it can be stored in polynomial space.

The lower bound is proved by reduction of the satisfiability problem for quantified Boolean formulas (QBFs), which is known to be PSPACE-complete. Let $\varphi=Q_{n} p_{n} \ldots Q_{0} p_{0} \varphi_{0}$ be a QBF, where each $Q_{i}$ is either $\forall$ or $\exists$, and $\varphi_{0}=c_{0} \wedge \cdots \wedge c_{m}$ is a propositional formula in CNF with $c_{i}=l_{0} \vee \cdots \vee l_{k}$, with each $l_{i}$ being either a variable $p_{j}$ or its negation $\neg p_{j}$, for $0 \leq j \leq n$. In our datalog ${ }_{n r} M T L$ program, we use the following propositional variables:

- $P_{0}, \ldots, P_{n}$ (to represent $p_{0}, \ldots, p_{n}$ from $\varphi$ );

- $\bar{P}_{0}, \ldots, \bar{P}_{n}$ (to represent $\neg p_{0}, \ldots, \neg p_{n}$ );

- $P_{0}^{0}, \ldots, P_{0}^{n}$ for $p_{0} ; P_{1}^{1}, \ldots, P_{1}^{n}$ for $p_{1}$, etc.; $P_{n}^{n}$ for $p_{n}$, and similarly for $\neg p_{i}$; 


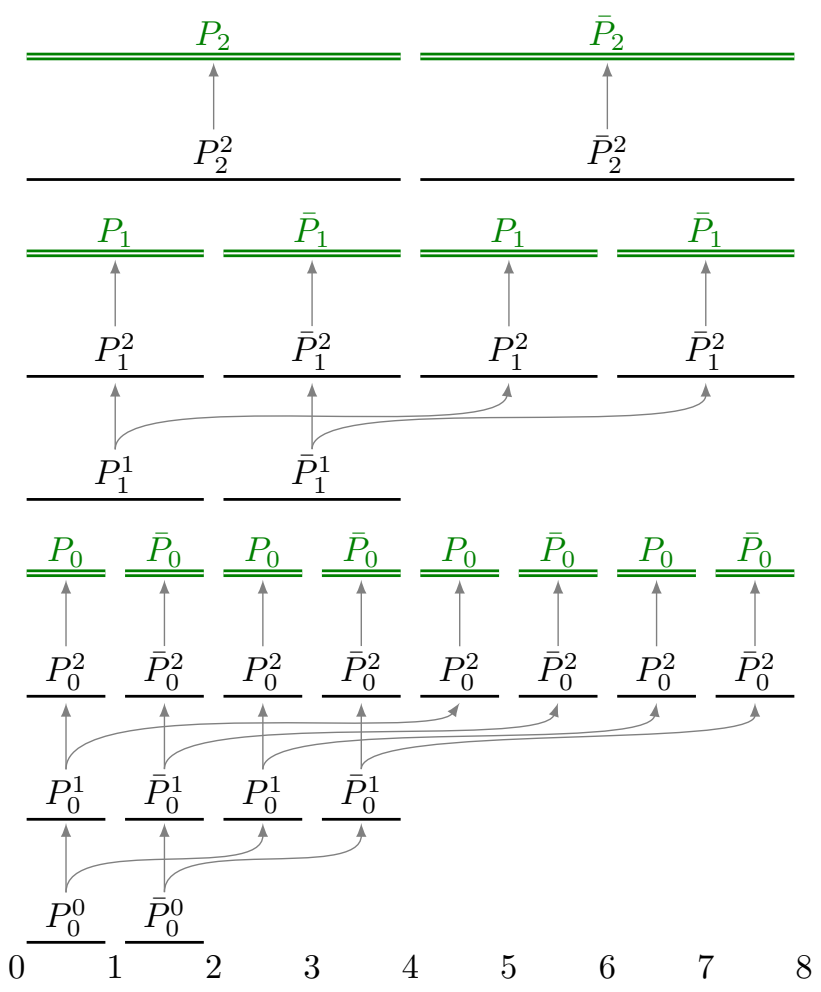

Figure 1: The canonical model for the proof of Theorem 13.

$-F_{0}, \ldots, F_{n+1}$

- $C_{0}, \ldots, C_{m}$ (to represent $\left.c_{0}, \ldots, c_{m}\right)$.

We first take a data instance $\mathcal{D}$ with the following facts:

$$
P_{i}^{i} @\left[0,2^{i}\right), \bar{P}_{i}^{i} @\left[2^{i}, 2^{i+1}\right), \quad \text { for } 0 \leq i \leq n .
$$

Starting from this data, we can generate all the truth-assignments for the variables $p_{0}, \ldots, p_{n}$ using the following rules, where $0 \leq i \leq n$ :

$$
\begin{aligned}
P_{i} & \leftarrow P_{i}^{n}, \quad \bar{P}_{i} \leftarrow \bar{P}_{i}^{n}, \\
P_{i}^{j+1} \leftarrow P_{i}^{j}, \quad \boxplus_{2^{j+1}} P_{i}^{j+1} & \leftarrow P_{i}^{j}, \quad \bar{P}_{i}^{j+1} \leftarrow \bar{P}_{i}^{j}, \quad \boxplus_{2^{j+1}} \bar{P}_{i}^{j+1} \leftarrow \bar{P}_{i}^{j}, \quad i \leq j<n .
\end{aligned}
$$

The canonical model for $\mathcal{D}$ and the rules above for the variables $p_{0}, p_{1}, p_{2}$ (thus, $n=2$ ) is shown in Fig. 1.

We then need the rules:

$$
\begin{aligned}
& C_{i} \leftarrow P_{j}, \quad p_{j} \text { occurs in } c_{i}, \\
& C_{i} \leftarrow \bar{P}_{j}, \quad \neg p_{j} \text { occurs in } c_{i}, \\
& F_{0} \leftarrow \bigwedge_{0 \leq i \leq m} C_{i},
\end{aligned}
$$


for $0 \leq i \leq m, 0 \leq j \leq n$. Note that $F_{0}$ will hold at the moments of time corresponding to the assignments that make $\varphi_{0}$ true. Further, we consider the formula $\varphi_{i}=Q_{i-1} p_{i-1} \ldots Q_{0} p_{0} \varphi_{0}$, for $1 \leq i \leq n+1$ (note that $\varphi_{n+1}=\varphi$ ), and provide rules that make $F_{i}$ true precisely at the moments of time corresponding to the assignments that make $\varphi_{i}$ true. We take

$$
\begin{aligned}
& \boxplus_{\left[0,2^{i}\right]} F_{i+1} \leftarrow F_{i} \wedge P_{i}, \quad \boxminus_{\left[0,2^{i}\right]} F_{i+1} \leftarrow F_{i} \wedge \bar{P}_{i}, \quad \text { if } Q_{i}=\exists, \\
& \boxplus_{\left[0,2^{i+1}\right)} F_{i+1} \leftarrow \boxplus_{\left[0,2^{i}\right)} P_{i} \wedge \boxplus_{\left[0,2^{i+1}\right)} F_{i}, \quad \text { if } Q_{i}=\forall,
\end{aligned}
$$

for $0 \leq i \leq n$, and, finally,

$$
\perp \leftarrow \boxplus_{\left[0,2^{n+1}\right)} F_{n+1} .
$$

All the rules above form the required data $\log _{n r} M T L$ program $\Pi$. We now prove that $\Pi$ is consistent with $\mathcal{D}$ iff $\varphi$ is not satisfiable. By Lemma 5 , it suffices to show that $F_{n+1} @\left[0,2^{n+1}\right) \in \mathfrak{C}_{\Pi, \mathcal{D}}$ iff $\varphi$ is satisfiable. For $(\Rightarrow)$, suppose $F_{n+1} @\left[0,2^{n+1}\right) \in \mathfrak{C}_{\Pi, \mathcal{D}}$. If $Q_{n}=\exists$ then, in view of (14), either $F_{n} @\left[0,2^{n}\right), P_{n} @\left[0,2^{n}\right) \in \mathfrak{C}_{\Pi, \mathcal{D}}$ or $F_{n} @\left[2^{n}, 2^{n+1}\right), \bar{P}_{n} @\left[2^{n}, 2^{n+1}\right) \in \mathfrak{C}_{\Pi, \mathcal{D}}$. If the first option holds, we show that $\varphi_{n}$ is satisfiable when $p_{n}$ is true; if the second option holds, we show that $\varphi_{n}$ is satisfiable when $p_{n}$ is false. Similarly, if $Q_{n}=\forall$, then by (15), we have $F_{n} @\left[0,2^{n}\right), P_{n} @\left[0,2^{n}\right) \in \mathfrak{C}_{\Pi, \mathcal{D}}$ and $F_{n} @\left[2^{n}, 2^{n+1}\right), \bar{P}_{n} @\left[2^{n}, 2^{n+1}\right) \in \mathfrak{C}_{\Pi, \mathcal{D}}$. In this case, we show that $\varphi_{n}$ is satisfiable when $p_{n}$ can be both false and true. To show that $F_{n} @\left[0,2^{n}\right), P_{n} @\left[0,2^{n}\right) \in \mathfrak{C}_{\Pi, \mathcal{D}}$ implies that $\varphi_{n}$ is satisfiable when $p_{n}$ is true (the other case is analogous and left to the reader), suppose $Q_{n-1}=\exists$. By (14), either $F_{n-1} @\left[0,2^{n-1}\right), P_{n-1} @\left[0,2^{n-1}\right) \in \mathfrak{C}_{\Pi, \mathcal{D}}$ or $F_{n} @\left[2^{n-1}, 2^{n}\right), \bar{P}_{n-1} @\left[2^{n-1}, 2^{n}\right) \in \mathfrak{C}_{\Pi, \mathcal{D}}$. (If $Q_{n-1}=\forall$, by (14) both of these options hold.) Therefore, to show that $\varphi$ is satisfiable, it now suffices to show that $(i) F_{n-1} @\left[0,2^{n-1}\right), P_{n-1} @\left[0,2^{n-1}\right) \in \mathfrak{C}_{\Pi, \mathcal{D}}$ implies that $\varphi_{n-1}$ is satisfiable when $p_{n}$ is true and $p_{n-1}$ is true; (ii) $F_{n-1} @\left[2^{n-1}, 2^{n}\right), \bar{P}_{n-1} @\left[2^{n-1}, 2^{n}\right) \in \mathfrak{C}_{\Pi, \mathcal{D}}$ implies that $\varphi_{n-1}$ is satisfiable when $p_{n}$ is true and $p_{n-1}$ is false. We only consider (i), leaving (ii) to the reader, and after applying the argument above $n$ times, will need to show that $(i) F_{0} @[0,1), P_{0} @[0,1) \in \mathfrak{C}_{\Pi, \mathcal{D}}$ implies that $\varphi_{0}$ is satisfiable when $p_{n}, \ldots, p_{1}$ and $p_{0}$ are all true; (ii) $F_{0} @[1,2), \bar{P}_{0} @[1,2) \in \mathfrak{C}_{\Pi, \mathcal{D}}$ implies that $\varphi_{0}$ is satisfiable when $p_{n}, \ldots, p_{1}$ are true while $p_{0}$ is false. That $(i)$ holds follows from (11)-(13), and similarly for (ii). This concludes the proof of $(\Rightarrow)$; the other direction is proved analogously.

Using the techniques of Artale, Kontchakov, Ryzhikov, and Zakharyaschev (2014), it can be shown that nonrecursive Horn fragment of LTL is P-complete. The same complexity can be derived from the work by Bresolin et al. (2017) for the nonrecursive Horn fragment of the Halpern-Shoham $\operatorname{logic} \mathcal{H S}$.

As we have just seen, the combined complexity of query answering drops from EXPSPACE for datalogMTL to PSPACE for datalog $\log _{n} M T L$. We now show that the data complexity drops to $\mathrm{AC}^{0}$, which is important for practical query answering using standard database systems. Note that this result is non-trivial in view of Theorem 9. The crux of the proof is encoding coalescing by FOformulas with $\forall$ (which is typically not needed for rewriting atemporal ontology-mediated queries).

Theorem 14. Consistency checking and answering datalog ${ }_{n r} M T L$ queries is in $A C^{0}$ for data complexity.

Proof. We only consider a propositional datalog ${ }_{n r} M T L$ program $\Pi$. The proof can be straightforwardly adapted to the case of arity $\geq 1$ by adding more (object) variables to the predicates 
used below. Let $N$ be a set of comprising numbers or $\infty,-\infty$. We use $N+r$ as a shorthand for $\{t+r \mid t \in N\}$ and similarly for $N-r$ (we assume that $t+\infty=\infty$ and $t-\infty=-\infty$ ). For a propositional variable $P$ in $\Pi$, we define two sets le $(P)$ and ri $(P)$ as follows:

- le $(P)=\operatorname{ri}(P)=\{0\}$ if there is no $P^{\prime}$ such that $P \lessdot P^{\prime}$;

- otherwise, le $(P)$ is the union of:

- $\bigcup_{i \in I}$ le $\left(P_{i}\right)$, for each $P \leftarrow \bigwedge_{i \in I} P_{i}$ in $\Pi$,

- le $\left(P_{2}\right)+r_{1} \cup \mathrm{ri}\left(P_{1}\right)$, for each $P \leftarrow P_{1} \mathcal{S}_{\left\langle r_{1}, r_{2}\right\rangle} P_{2}$ in $\Pi$,

- le $\left(P_{2}\right)-r_{2} \cup \operatorname{le}\left(P_{1}\right)$, for each $P \leftarrow P_{1} \mathcal{U}_{\left\langle r_{1}, r_{2}\right\rangle} P_{2}$ in $\Pi$,

- le $\left(P_{1}\right)+r_{2}$, for each $P \leftarrow \boxminus_{\left\langle r_{1}, r_{2}\right\rangle} P_{1}$ in $\Pi$,

- le $\left(P_{1}\right)-r_{1}$, for each $P \leftarrow \boxplus_{\left\langle r_{1}, r_{2}\right\rangle} P_{1}$ in $\Pi$,

and $\mathrm{ri}(P)$ is the union of:

- $\bigcup_{i \in I} \mathrm{ri}\left(P_{i}\right)$, for each $P(\tau) \leftarrow \bigwedge_{i \in I} P_{i}$ in $\Pi$,

- ri $\left(P_{2}\right)+r_{2} \cup \operatorname{ri}\left(P_{1}\right)$, for each $P \leftarrow P_{1} \mathcal{S}_{\left\langle r_{1}, r_{2}\right\rangle} P_{2}$ in $\Pi$,

- ri $\left(P_{2}\right)-r_{1} \cup \operatorname{le}\left(P_{1}\right)$, for each $P \leftarrow P_{1} \mathcal{U}_{\left\langle r_{1}, r_{2}\right\rangle} P_{2}$ in $\Pi$,

- ri $\left(P_{1}\right)+r_{1}$, for each $P \leftarrow \boxminus_{\left\langle r_{1}, r_{2}\right\rangle} P_{1}$ in $\Pi$,

- ri $\left(P_{1}\right)-r_{2}$, for each $P \leftarrow \boxplus_{\left\langle r_{1}, r_{2}\right\rangle} P_{1}$ in $\Pi$.

Using an argument that is similar to the proof of Lemma 11, one can show the following:

Lemma 15. For any datalog ${ }_{n r} M T L$ program $\Pi$, any data instance $\mathcal{D}$, and any $P @\left\langle t_{1}, t_{2}\right\rangle \in \mathfrak{C}_{\Pi, \mathcal{D}}$,

- $t_{1}=t_{1}^{\prime}+n_{1}$, for some $n_{1} \in \operatorname{le}(P)$ and some $t_{1}^{\prime}$ such that $P^{\prime}\left[t_{1}^{\prime}, t_{1}^{\prime}\right] \in \mathcal{D}$ or $P^{\prime}\left(t_{1}^{\prime}, s_{2}\right) \in \mathcal{D}$,

- $t_{2}=t_{2}^{\prime}+n_{2}$, for some $n_{2} \in \mathrm{ri}(P)$ and some $t_{2}^{\prime}$ such that $P^{\prime}\left[t_{2}^{\prime}, t_{2}^{\prime}\right] \in \mathcal{D}$ or $P^{\prime}\left(s_{1}, t_{2}^{\prime}\right) \in \mathcal{D}$.

In view of Lemma 15, we can prove Theorem 14 by constructing FO-formulas $\varphi_{P}^{\langle m, n\rangle}(x, y)$ with $m \in \operatorname{le}(P)$ and $n \in \operatorname{ri}(P)$ such that, for any data instance $\mathcal{D}$,

$$
P @\left\langle t_{1}+m, t_{2}+n\right\rangle \in \mathfrak{C}_{\Pi, \mathcal{D}} \quad \text { iff } \quad \mathfrak{A}_{\mathcal{D}} \models \varphi_{P}^{\langle m, n\rangle}\left(t_{1}, t_{2}\right),
$$

where $\mathfrak{A}_{\mathcal{D}}$ is the FO-structure defined below. To slightly simplify presentation (and without much loss of generality), we assume that all numbers in num $(\mathcal{D})$ are positive, and set

$$
\mathfrak{A}_{\mathcal{D}}=\left(\Delta,<, P_{1}^{[]}, P_{1}^{()}, \ldots, P_{l}^{[]}, P_{l}^{()}, \text {bit }^{\text {in }}, \text { bit }^{\text {fr }}\right),
$$

where

- $\Delta$ is a set of $(\ell+1)$-many elements strictly linearly ordered by $<, \ell$ is the maximum of the number of distinct timestamps in $\mathcal{D}$ and the number of bits in the longest binary fraction in $\mathcal{D}$ (excluding the binary point); for simplicity, we assume that $\Delta=\{0, \ldots, \ell\},<$ is the natural order, and denote by $\bar{n}$ the $n$th fraction in $(\operatorname{num}(\mathcal{D}),<)$, counting from 0 ; 
- $P_{i}^{[]}(n, n)$ holds in $\mathfrak{A}_{\mathcal{D}}$ iff $P_{i} @[\bar{n}, \bar{n}] \in \mathcal{D}$ and $P_{i}^{()}(n, m)$ holds in $\mathfrak{A}_{\mathcal{D}}$ iff $P_{i} @(\bar{n}, \bar{m}) \in \mathcal{D}$, for any $P_{i}$ occurring in $\mathcal{D}$;

- for $\bar{n} \neq \infty$ bit $^{i n}(n, i, 0)$ bit $^{f r}(n, i, 0)$ ) holds in $\mathfrak{A}_{\mathcal{D}}$ iff the $i$ th bit of the integer (respectively, fractional) part of $\bar{n}$ is 0 , and $\operatorname{bit}^{i n}(n, i, 1)$ (bit ${ }^{f r}(n, i, 1)$ ), for $i \in \Delta$, holds in $\mathfrak{A}_{\mathcal{D}}$ iff the $i$ th bit of the integer (respectively, fractional) part of $\bar{n}$ is 1 (as usual, we start counting bits from the least significant one);

- for $\bar{n}=\infty, \operatorname{bit}^{i n}(n, i, 1)$ and $\operatorname{bit}^{f r}(n, i, 1)$ for all $i \in \Delta$.

For example, the data instance $\mathcal{D}=\{P[110.001,110.001], P(10000, \infty)\}$ is given as the FO structure

$$
\mathfrak{A}_{\mathcal{D}}=\left(\Delta,<, P^{[]}, P^{()}, \text {bit }^{\text {in }}, \text { bit }^{f r}\right),
$$

where $\Delta=\{0, \ldots, 6\}, P^{[]}=\{(0,0)\}, P^{()}=\{(1,2)\}$, and

$$
\begin{aligned}
\text { bit }^{i n}= & \{(0,0,0),(0,1,1),(0,2,1)\} \cup\{(0, i, 0) \mid 3 \leq i \leq 6\} \cup \\
& \{(1, i, 0) \mid 0 \leq i \leq 3\} \cup\{(1,4,1)\} \cup\{(1,5,0)\} \cup\{(1,6,0)\} \cup \\
& \{(2, i, 1) \mid 0 \leq i \leq 6\} . \\
\text { bit }^{f r}= & \{(0,4,1)\} \cup\{(0, i, 0) \mid 0 \leq i \leq 6, i \neq 4\} \cup \\
& \{(1, i, 0) \mid 0 \leq i \leq 6\} \cup \\
& \{(2, i, 1) \mid 0 \leq i \leq 6\} .
\end{aligned}
$$

To construct the required $\varphi_{P}^{\langle m, n\rangle}(x, y)$, suppose that we have FO-formulas

- coal $_{P}^{\langle m, n\rangle}(x, y)$ saying that $P @\langle x+m, y+n\rangle$ is added to $\mathfrak{C}_{\Pi, \mathcal{D}}$ by an application of the rule (coal);

- $\psi_{P}^{\langle m, n\rangle}(x, y)$ saying that

either $P @\langle x+m, y+n\rangle$ is added to $\mathfrak{C}_{\Pi, \mathcal{D}}$ because it belongs to the given data instance (in which case we can assume that $m=n=0$, and \langle\rangle is either () or []),

or $P @\langle x+m, y+n\rangle$ is added to $\mathfrak{C}_{\Pi, \mathcal{D}}$ as a result of an application of one of the 'logical' rules.

In this case we can set

$$
\varphi_{P}^{\langle m, n\rangle}(x, y)=\psi_{P}^{\langle m, n\rangle}(x, y) \vee \operatorname{coal}_{P}^{\langle m, n\rangle}(x, y)
$$

Using the predicate is s,b $_{a, b}$ which is $\top$ if $a=b$ and $\perp$ otherwise, we can define $\psi_{P}^{\langle m, n\rangle}(x, y)$ as a disjunction of the following formulas:

$$
\begin{aligned}
& \text { - is } \left.{ }_{\langle,[} \wedge \text { is }\right\rangle_{,]} \wedge \text { is }_{m, 0} \wedge \text { is }_{n, 0} \wedge P^{[]}(x, y) ; \\
& \text { - is } \left.\left.{ }_{\langle,(} \wedge \text { is }\right\rangle,\right) \\
& \text { - for every } P \leftarrow \text { is }_{m, 0} \wedge \text { is }_{n, 0} \wedge P^{()}(x, y) ; \\
& P_{i} \text { in } \Pi,
\end{aligned}
$$




$$
\begin{aligned}
& \exists x_{1}, y_{1}, \ldots, x_{k}, y_{k} \bigvee_{\substack{m_{1} \in \operatorname{le}\left(P_{1}\right) \\
n_{1} \in \operatorname{ri}\left(P_{1}\right) \\
\left\lceil_{1} \in\left\{[,(\},\rceil_{1} \in\{],\right)\right\}}}\left(\varphi _ { P _ { 1 } } ^ { \lceil _ { 1 } m _ { 1 } , n _ { 1 } \rceil _ { 1 } } ( x _ { 1 } , y _ { 1 } ) \wedge \cdots \wedge \bigvee _ { \substack { m _ { k } \in \operatorname { l e } ( P _ { k } ) \\
n _ { k } \in \operatorname { r i } ( P _ { k } ) \\
\Gamma _ { k } \in \{ [ , ( \} , \rceil _ { k } \in \{ ] , ) \} } } \left(\varphi_{P_{k}}^{\left\lceil_{k} m_{k}, n_{k}\right\rceil_{k}}\left(x_{k}, y_{k}\right) \wedge\right.\right. \\
& \left.\left.\operatorname{inter}_{\left.\left\lceil_{1} m_{1}, n_{1}\right\rceil_{1}, \ldots, \Gamma_{k} m_{k}, n_{k}\right\rceil_{k}}^{\langle m, n\rangle}\left(x, y, x_{1}, y_{1}, \ldots, x_{k}, y_{k}\right)\right) \ldots\right) \text {, }
\end{aligned}
$$

where inter ${ }_{\left\lceil_{1} m_{1}, n_{1}\right\rceil_{1}, \ldots,\left\lceil_{k} m_{k}, n_{k}\right\rceil_{k}}^{\langle m, n\rangle}\left(x, y, x_{1}, y_{1}, \ldots, x_{k}, y_{k}\right)$ says that $\langle x+m, y+n\rangle$ is an intersection of $\left\lceil_{1} x_{1}+m_{1}, y_{1}+n_{1}\right\rceil_{1}, \ldots,\left\lceil_{k} x_{k}+m_{k}, y_{k}+n_{k}\right\rceil_{k}$ (this formula can easily be defined in terms of the predicates $x+m=y+n$ and $x+m<y+n$ given below);

- for every $P \leftarrow P_{1} \mathcal{S}_{\varrho} P_{2}$ in $\Pi$, the formula $\sigma_{\varrho, P, P_{1}, P_{2}}^{\langle m, n\rangle}(x, y)$ saying that $\langle x+m, y+n\rangle$ is $\left(\left(\iota_{1}^{c} \cap \iota_{2}\right)-{ }^{o} \varrho\right) \cap \iota_{1}^{c}$ for some $\iota_{1}$ and $\iota_{2}$, where $P_{1}$ and $P_{2}$ hold, respectively (we give a definition of $\sigma_{\varrho, P, P_{1}, P_{2}}^{\langle m, n\rangle}(x, y)$ in the appendix);

- analogous formulas encoding the relevant operations on intervals for the other temporal operators.

The formula coal ${ }_{P}^{\langle m, n\rangle}(x, y)$ is defined as follows:

$$
\operatorname{coal}_{P}^{\langle m, n\rangle}(x, y)=\forall z \bigwedge_{l \in \operatorname{le}(P) \cup \operatorname{ri}(P)}\left((x+m \leq z+l) \wedge(z+l \leq y+m) \rightarrow \operatorname{nogap}_{P,\langle m, n\rangle}^{l}(z, x, y)\right),
$$

where $\operatorname{nogap}_{P,\langle m, n\rangle}^{l}(z, x, y)$ is the formula

$$
\begin{aligned}
& \exists x_{1}, y_{1}, x_{2}, y_{2}, x_{3}, y_{3} \bigvee_{\substack{\left.m_{1} \in \operatorname{le}(P) \\
n_{1} \in \operatorname{ri}(P) \\
\Gamma_{1} \in\left\{[,(\},\rceil_{1} \in\{],\right)\right\}}}\left(\psi_{P}^{\left\lceil{ }_{1} m_{1}, n_{1}\right\rceil_{1}}\left(x_{1}, y_{1}\right) \wedge \operatorname{sub}_{\langle m, n\rangle}^{\left\lceil_{1} m_{1}, n_{1}\right\rceil_{1}}\left(x_{1}, y_{1}, x, y\right) \wedge\right. \\
& \bigvee_{\substack{\left.m_{2} \in \operatorname{le}(P) \\
n_{2} \in \operatorname{ri}(P) \\
{ }_{2} \in\left\{[,(\},\rceil_{2} \in\{],\right)\right\}}}\left(\psi_{P}^{\left\lceil_{2} m_{2}, n_{2}\right\rceil_{2}}\left(x_{2}, y_{2}\right) \wedge \operatorname{sub}_{\langle m, n\rangle}^{\left\lceil_{2} m_{2}, n_{2}\right\rceil_{2}}\left(x_{2}, y_{2}, x, y\right) \wedge\right. \\
& \begin{array}{c}
\left(\left(x_{1}+m_{1}<z+l<y_{1}+n_{1}\right) \vee\right. \\
\left.\left(x_{1}+m_{1}<y_{1}+l_{1}=z+l=x_{2}+m_{2}<y_{2}+n_{2}\right) \wedge \text { is }_{\rceil_{1},\right]} \vee \text { is }_{{ }_{2},[}\right) \vee \\
\bigvee_{\substack{m_{3} \in \operatorname{le}(P) \\
n_{3} \in \operatorname{ri}(P)}}\left(\psi_{P}^{\left[m_{3}, n_{3}\right]}\left(x_{3}, y_{3}\right) \wedge \operatorname{sub}_{\langle m, n\rangle}^{\left[m_{3}, n_{3}\right]}\left(x_{3}, y_{3}, x, y\right) \wedge\right. \\
\quad\left(\left(x_{3}+m_{3}=y_{3}+n_{3}=z+l=x+m=x_{1}+m_{1}<y_{1}+n_{1}\right) \vee\right. \\
\quad\left(x_{1}+m_{1}<y_{1}+n_{1}=z+l=y+n=x_{3}+m_{3}=y_{3}+n_{3}\right) \vee \\
\left.\left.\left.\left.\left.x_{1}+m_{1}<y_{1}+l_{1}=z+l=x_{3}+m_{3}=y_{3}+n_{3}=x_{2}+m_{2}<y_{2}+n_{2}\right)\right]\right)\right)\right)
\end{array}
\end{aligned}
$$

and $\operatorname{sub}_{\langle m, n\rangle}^{\left\lceil m^{\prime}, n^{\prime}\right\rceil}\left(x^{\prime}, y^{\prime}, x, y\right)$ says that $\left\lceil x^{\prime}+m^{\prime}, y^{\prime}+n^{\prime}\right\rceil$ is a subinterval of $\langle x+m, y+n\rangle$. Intuitively, $\operatorname{nogap}_{P,\langle m, n\rangle}^{l}(z, x, y)$ says that around the time instant $z+l$ (that is, to the left and right of it as well 
Case (18)

Case (19)

Case (20)

Case (21)

Case (22)

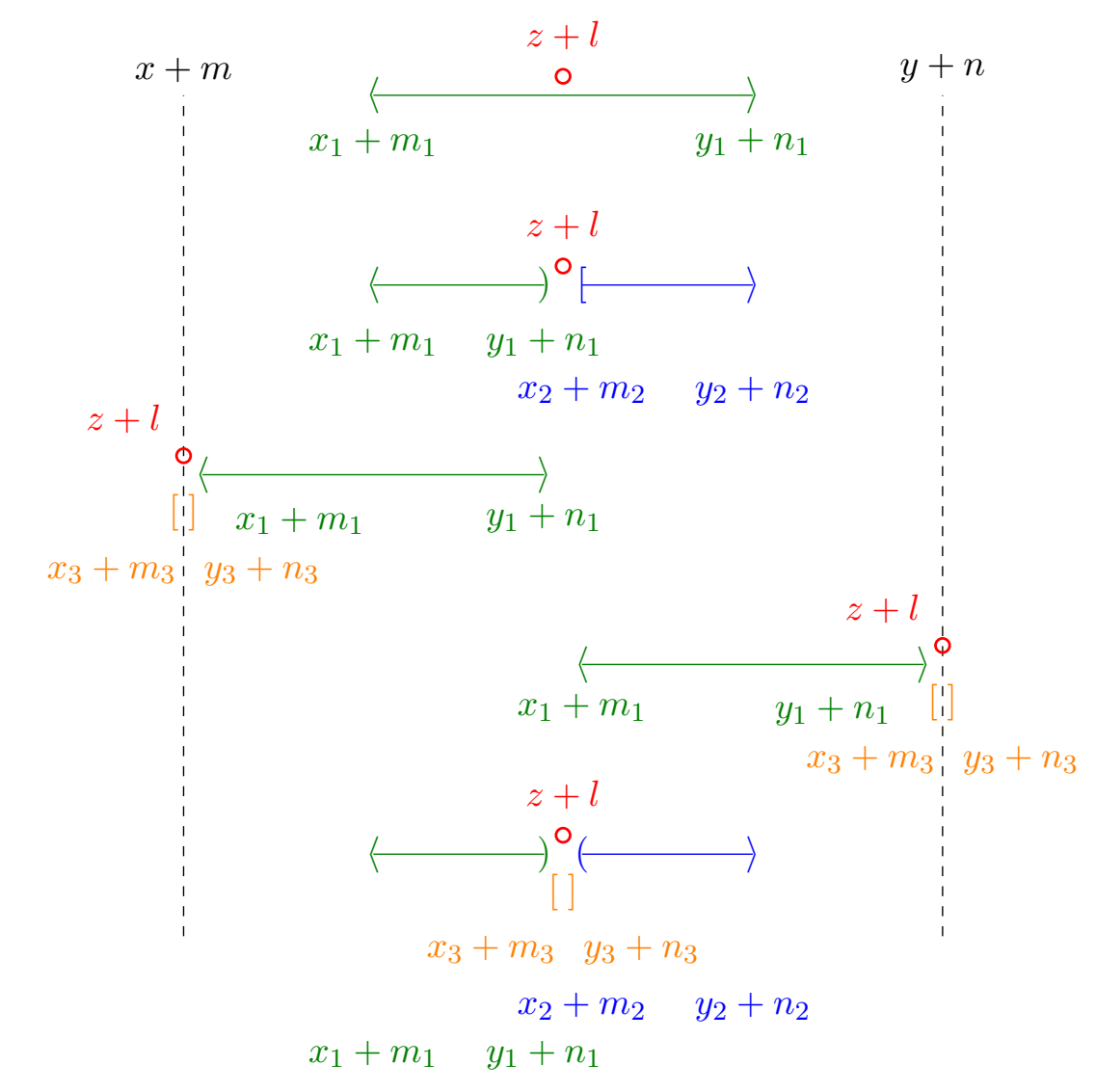

Figure 2: Five cases of the formula nogap $p_{P,\langle m, n\rangle}^{l}(z, x, y)$.

as at $z+l$ itself), their is no subinterval of $\langle x+m, y+n\rangle$ that is not covered by $P$. The five cases considered in the formula nogap ${ }_{P,\langle m, n\rangle}^{l}(z, x, y)$ are illustrated in Fig. 2.

When evaluating $\varphi^{\langle m, n\rangle}(x, y)$ over $\mathfrak{A}_{\mathcal{D}}$, we need to compute the truth-values of $x+m=y+n$ and $x+m<y+n$ (for fixed $m$ and $n$ ). We regard the former as a formula with the predicates bit ${ }^{i n}$, $\mathrm{bit}^{f r}$ and $<$ that is true just in case $x=y+(n-m)$ if $n \geq m$, and $y=x+(m-n)$ otherwise. We provide a definition of $x=y+c$, for a positive $c$, in the appendix. A formula expressing $x+m<y+n$ is constructed similarly and left to the reader.

Finally, we show how the formulas $\varphi_{P}^{\langle m, n\rangle}(x, y)$ defined above can be used to check whether an interval $\iota=\left\langle\iota_{b}, \iota_{e}\right\rangle$ is a certain answer to $(\Pi, P @ x)$ over $\mathcal{D}$. As follows from Lemma 15, if $\perp @\left\lceil t_{1}, t_{2}\right\rceil \in \mathfrak{C}_{\Pi, \mathcal{D}}$ then, for some $m \in \mathrm{le}(\perp), n \in \mathrm{ri}(\perp)$ and some numbers $t_{1}^{\prime}, t_{2}^{\prime} \in \operatorname{num}(\mathcal{D})$ such that $t_{1}^{\prime}\left(t_{2}^{\prime}\right)$ occurs as the left (right) end of some interval, we have $t_{1}=t_{1}^{\prime}+m$ and $t_{2}=t_{2}^{\prime}+n$. Take the structure $\mathfrak{A}_{\mathcal{D}}^{\iota}$ that extends $\mathfrak{A}_{\mathcal{D}}$ with the numbers $\iota_{b}$ and $\iota_{e}$. By (16), $\iota$ is a certain answer to 
$(\Pi, P @ x)$ over $\mathcal{D}$ iff the formula

$$
\begin{aligned}
& \exists x, y \bigvee_{\substack{m \in \operatorname{le}(\perp) \\
n \in \operatorname{ri}(\perp)}} \varphi_{\perp}^{\lceil m, n\rceil}(x, y) \vee \\
& \exists x, y, x_{1}, y_{1} \bigvee_{\substack{m_{1} \in \operatorname{le}(\perp) \\
n_{1} \in \operatorname{ri}(\perp) \\
\left\lceil_{1} \in\left\{[,(\},\rceil_{1} \in\{],\right)\right\}}}\left(\varphi_{P}^{\lceil 1 m, n\rceil_{1}}\left(x_{1}, y_{1}\right) \wedge \operatorname{sub}_{\left\lceil_{1} m, n\right\rceil_{1}}^{\langle 0,0\rangle}\left(x, y, x_{1}, y_{1}\right) \wedge\left(x=\iota_{b}\right) \wedge\left(y=\iota_{e}\right)\right)
\end{aligned}
$$

holds true in $\mathfrak{A}_{\mathcal{D}}^{\iota}$.

\section{Implementing Datalog ${ }_{n r} M T L$}

Unfortunately, the (data independent) FO-rewriting (23) turns out to be impractical because of the universal quantifier used for coalescing in (17). It is well known that $\forall$ is implemented in SQL as $\neg \exists \neg$ resulting in suboptimal performance in general. Having experimented with a few different approaches, we decided to use a materialisation (bottom-up) technique. In this section, we first present a bottom-up algorithm whose worst-case running time is linear in the number of intervals of an input data instance $\mathcal{D}$, under a practically motivated assumption that the order of occurrence of the intervals in $\mathcal{D}$ coincides with the natural temporal order on those intervals. Then we describe how our algorithm can be implemented in SQL (with views). In particular, we consider two alternative implementations of coalescing in SQL.

\subsection{Bottom-up Algorithm}

We first introduce some notation and obtain a few results about temporal tables $T$ with column names $\operatorname{attr}_{1}, \ldots$, attr $_{m}$, Ipar, ledge, redge, rpar. A temporal table with $m=0$ will be called purely temporal. We refer to the $i$-th row of $T$ as $T[i]$, to the value of the column $\operatorname{attr}_{j}$ in the $i$-th row as $T\left[i, \operatorname{attr}_{j}\right]$, and set $T\left[i, \operatorname{attr}_{j}, \ldots, \operatorname{attr}_{k}\right]=\left(T\left[i, \operatorname{attr}_{j}\right], \ldots, T\left[i, \operatorname{attr}_{k}\right]\right)$. We assume that the columns ledge and redge store timestamps or special values for $\infty,-\infty$, Ipar stores [ or (, and rpar stores ] or $)$. Define an order $\prec$ on intervals by taking $\left\langle t_{1}, t_{2}\right\rangle \prec\left\lceil s_{1}, s_{2}\right\rceil$ iff one of the following conditions holds:

$$
\begin{aligned}
& -t_{1}<s_{1} ; \\
& -t_{1}=s_{1},\langle\text { is }[\text {, and }\lceil\text { is }(; \\
& -t_{1}=s_{1},\left\langle\text { and } \left\lceil\text { are the same, and } t_{2}<s_{2} ;\right.\right. \\
& -t_{1}=s_{1},\left\langle\text { and }\left\lceil\text { are the same, } t_{2}=s_{2},\right\rangle \text { is }\right) \text {, and }\lceil\text { is }] .
\end{aligned}
$$

It should be clear that $\prec$ is a strict linear order on the set of all intervals. For example, we have $[3,8) \prec[4,7) \prec(4,6) \prec(4,7) \prec(4,7]$. (In fact, the results of this section will work with any other linear order over intervals.) We write $T[i$, lpar, ledge, redge, rpar $] \prec T^{\prime}[j$, Ipar, ledge, redge, rpar $]$ to say that the interval defined by the $i$ th row of a temporal table $T \prec$-precedes the interval given by the $j$ th row of a temporal table $T^{\prime}$. 
We make the following temporal ordering assumption (or TOA), for any temporal table $T$ with $m$ attributes:

$$
\begin{aligned}
\text { if } T\left[i, \operatorname{attr}_{1}, \ldots, \operatorname{attr}_{m}\right]=T\left[j, \operatorname{attr}_{1}, \ldots, \operatorname{attr}_{m}\right] \text { and } i<j, \\
\\
\text { then } T[i, \text { Ipar, ledge, redge, } \operatorname{rpar}] \preceq T[j, \text { Ipar, ledge, redge, rpar }] .
\end{aligned}
$$

For a purely temporal table $T$, this assumption means that the rows of $T$ respect $\preceq$.

Let $T\left[\operatorname{attr}_{j}, \ldots, \operatorname{attr}_{k}\right]$ be the projection of $T$ on the columns $\operatorname{attr}_{j}, \ldots, \operatorname{attr}_{k}$ that keeps only distinct tuples. We define $|T|_{o}$ to be the cardinality of $T\left[\operatorname{attr}_{1}, \ldots\right.$, attr $\left.{ }_{m}\right]$ and $|T|_{t}$ to be the cardinality of $T$ [lpar, ledge, redge, rpar]. The first measure estimates how large the table is in terms of individual constants, while the second measure concerns the number of timepoints. For the tables of extensional predicates in our use-cases, $|T|_{o}$ is much smaller than $|T|_{t}$.

We say that a table $T$ is coalesced if it does not contain distinct tuples $\left(c_{1}, \ldots, c_{m},\left\langle, t_{1}, t_{2},\right\rangle\right)$ and $\left(c_{1}, \ldots, c_{m},\left\lceil, t_{1}^{\prime}, t_{2}^{\prime},\right\rceil\right)$ such that $\left\langle t_{1}, t_{2}\right\rangle \cap\left\lceil t_{1}^{\prime}, t_{2}^{\prime}\right\rceil \neq \emptyset$. For a tuple of individual constants $\left(c_{1}, \ldots, c_{m}\right)$, let $T_{c_{1}, \ldots, c_{m}}$ be the set of all intervals $\left\langle t_{1}, t_{2}\right\rangle$ such that $\left(c_{1}, \ldots, c_{m},\left\langle, t_{1}, t_{2},\right\rangle\right)$ occurs in $T$. For a set $\mathcal{I}$ of intervals, we then denote by coalesce $(\mathcal{I})$ the (minimal) set of intervals that results from coalescing $\mathcal{I}$. Finally, a coalescing of $T$ is a minimal table, $T^{*}$, with the same columns as $T$ such that the following condition holds:

(coalesce) for any $\left(c_{1}, \ldots, c_{m}\right)$ in $T\left[\operatorname{attr}_{1}, \ldots, \operatorname{attr}_{m}\right]$ and $\left\langle t_{1}, t_{2}\right\rangle$ in coalesce $\left(T_{c_{1}, \ldots, c_{m}}\right)$, there exists $\left(c_{1}, \ldots, c_{m},\left\langle t_{1}, t_{2}\right\rangle\right)$ in $T^{*}$.

Clearly, $T^{*}$ is a coalesced table.

Lemma 16. Suppose a table $T$ satisfies TOA. Then its coalescing $T^{*}$ satisfying TOA and such that $\left|T^{*}\right|_{o}=|T|_{o}$ and $\left|T^{*}\right|_{t} \leq|T|_{t}$ can be computed in time $O\left(|T|_{o}^{2} \times|T|_{t}\right)$.

Proof. Consider first a purely temporal table $S$ that satisfies temporal ordering. There is a simple linear-time algorithm to produce a coalesced table $S^{*}$ that also satisfies temporal ordering. Indeed, initially we set $\langle b, e\rangle=S[0$, Ipar, ledge, redge, rpar $]$. In a loop, we take each $\left\lceil t_{1}, t_{2}\right\rceil=$ $S[i$, Ipar, ledge, redge, rpar $]$ (clearly, $\langle b, e\rangle \prec\left\lceil t_{1}, t_{2}\right\rceil$ ). If $\left\lceil t_{1}, t_{2}\right\rceil$ and $\langle b, e\rangle$ are disjoint, we add $\langle b, e\rangle$ to $S^{*}$ and set $\langle b, e\rangle=\left\lceil t_{1}, t_{2}\right\rceil$. If they are not disjoint, we set $\langle b, e\rangle=\left\lceil t_{1}, t_{2}\right\rceil \cup\langle b, e\rangle$ and move on. It is easily checked that the resulting table $S^{*}$ is as required. Below, we refer to this algorithm as an imperative coalescing algorithm.

It only remains to explain how the algorithm above can be applied to $T$ in order to obtain the required complexity. Note that $|T| \leq|T|_{o} \times|T|_{t}$ and we can construct $|T|_{o}$-many separate tables $T_{c_{1}, \ldots, c_{m}}$, for each $\left(c_{1}, \ldots, c_{m}\right)$, in time $|T| \times|T|_{o}$. Then, we can apply the algorithm described above to each $T_{c_{1}, \ldots, c_{m}}$ in time $|T|_{t}$ and merge the results. Therefore, the overall running time is $|T| \times|T|_{o}+|T|_{t} \times|T|_{o}=O\left(|T|_{o}^{2} \times|T|_{t}\right)$.

Before presenting our query answering algorithm, we determine the complexity of computing temporal joins. Let $T$ be a table with attributes $\operatorname{attr}_{1}, \ldots$, attr $m$, Ipar, ledge, redge, rpar and let $T^{\prime}$ be a table with attributes attr ${ }_{1}^{\prime}, \ldots$, attr ${ }_{n}^{\prime}$, Ipar, ledge, redge, rpar. A temporal join of $T$ and $T^{\prime}$ is a table $T^{\prime \prime}$ with attributes attr ${ }_{1}^{\prime \prime}, \ldots$, attr ${ }_{k}^{\prime \prime}$, ledge, redge, rpar such that

$$
\left\{\operatorname{attr}_{1}^{\prime \prime}, \ldots, \operatorname{attr}_{k}^{\prime \prime}\right\}=\left\{\operatorname{attr}_{1}, \ldots, \operatorname{attr}_{m}\right\} \cup\left\{\operatorname{attr}_{1}^{\prime}, \ldots, \operatorname{attr}_{n}^{\prime}\right\}
$$

and $\left(c_{1}^{\prime \prime}, \ldots, c_{k}^{\prime \prime},\left\langle, t_{1}^{\prime \prime}, t_{2}^{\prime \prime},\right\rangle\right)$ is in $T^{\prime \prime}$ iff there exist two tuples $\left(c_{1}, \ldots, c_{m},\left\lceil, t_{1}, t_{2},\right\rceil\right)$ from $T$ and $\left(c_{1}^{\prime}, \ldots, c_{n}^{\prime},\left\lfloor, t_{1}^{\prime}, t_{2}^{\prime},\right\rfloor\right)$ from $T^{\prime}$ satisfying the following conditions: 
$-c_{i}^{\prime \prime}=c_{j}$, for all $i, j$ such that $\operatorname{attr}_{i}^{\prime \prime}=\operatorname{attr}_{j}$;

- $c_{i}^{\prime \prime}=c_{j}^{\prime}$, for all $i, j$ such that $\operatorname{attr}_{i}^{\prime \prime}=\operatorname{attr}_{j}^{\prime}$;

- $\left\lceil t_{1}, t_{2}\right\rceil \cap\left\lfloor t_{1}^{\prime}, t_{2}^{\prime}\right\rfloor \neq \emptyset$ and $\left\langle t_{1}^{\prime \prime}, t_{2}^{\prime \prime}\right\rangle=\left\lceil t_{1}, t_{2}\right\rceil \cap\left\lfloor t_{1}^{\prime}, t_{2}^{\prime}\right\rfloor$.

Lemma 17. If $T, T^{\prime}$ satisfy TOA, then a temporal join $T^{\prime \prime}$ of $T$ and $T^{\prime}$ satisfying TOA and such that $\left|T^{\prime \prime}\right|_{o} \leq|T|_{o} \times\left|T^{\prime}\right|_{o},\left|T^{\prime \prime}\right|_{t} \leq|T|_{t}+\left|T^{\prime}\right|_{t}$ can be computed in time $O\left(|T|_{o}^{2} \times\left|T^{\prime}\right|_{o}^{2} \times\left(|T|_{t}+\left|T^{\prime}\right|_{t}\right)\right)$.

Proof. We first give an algorithm for computing the temporal join of purely temporal tables $S$ and $S^{\prime}$. We assume that these tables are coalesced (which can be done in time $O(|S|)$ and $O\left(\left|S^{\prime}\right|\right)$ ). The algorithm works starting from the first tuples $S[i]$ and $S^{\prime}\left[i^{\prime}\right]$ of the tables. If $S[i] \cap S^{\prime}\left[i^{\prime}\right] \neq \emptyset$, we write $S[i] \cap S^{\prime}\left[i^{\prime}\right]$ to the output table $S^{\prime \prime}$. Then, if $S[i+1] \succeq S^{\prime}\left[i^{\prime}+1\right]$, we set $i^{\prime}:=i^{\prime}+1$ (without changing $i$ ); otherwise, $i:=i+1$. We iterate until we have considered all the tuples in both tables. Clearly, computing the full $S^{\prime \prime}$ requires time $O\left(|S|+\left|S^{\prime}\right|\right)$.

The complete algorithm for the tables $T$ and $T^{\prime}$ will first, similarly to the argument of Lemma 16, produce $|T|_{o}$-many purely temporal tables $T_{c_{1}, \ldots, c_{m}}$, for each $\left(c_{1}, \ldots, c_{m}\right)$ occurring in $T$. Note that $\left|T_{c_{1}, \ldots, c_{m}}\right| \leq|T|_{t}$ for each of those tables. In the same way, we produce $\left|T^{\prime}\right|_{o}$ purely temporal tables $T_{c_{1}^{\prime}, \ldots, c_{n}^{\prime}}^{\prime}$, for each $\left(c_{1}^{\prime}, \ldots, c_{n}^{\prime}\right)$ occurring in $T^{\prime}$. It remains to apply the temporal join algorithm described above to all pairs of tables $T_{c_{1}, \ldots, c_{m}}$ and $T_{c_{1}^{\prime}, \ldots, c_{n}^{\prime}}^{\prime}$, which can be done in the required time.

Another operation on temporal tables we need is projection. Let $T$ be a table with column names as above and let $\left\{\operatorname{attr}_{1}^{\prime}, \ldots\right.$, attrr $\left.{ }_{n}^{\prime}\right\} \subseteq\left\{\operatorname{attr}_{1}, \ldots\right.$, attr $\left.m\right\}$. A projection of $T$ on $\operatorname{attr}_{1}^{\prime}, \ldots$, attr ${ }_{n}^{\prime}$ is a table with columns attr ${ }_{1}^{\prime}, \ldots$, attr ${ }_{n}^{\prime}$, Ipar, ledge, redge, rpar containing all $\left(c_{1}^{\prime}, \ldots, c_{n}^{\prime},\left\langle t_{1}, t_{2}\right\rangle\right)$ such that some $\left(c_{1}, \ldots, c_{m},\left\langle t_{1}, t_{2}\right\rangle\right)$ is in $T$ and $c_{i}^{\prime}=c_{j}$ whenever $\operatorname{attr}_{i}^{\prime}=\operatorname{attr}_{j}$. As we have to preserve the temporal order, our algorithm for computing projections requires some attention. To show that a naïve projection does not preserve the temporal order, consider a table $T$ with two tuples $(a,[, 1,1]$, and $(b,[, 0,0]$,$) , which satisfies our temporal order assumption. The projection of T$ that removes the first column results is the table with two tuples $([, 1,1]$,$) and ([, 0,0]$,$) , which is not ordered.$

Lemma 18. If $T$ satisfies TOA, then a projection of $T$ satisfying TOA can be computed in time $O\left(|T|_{o}^{2} \times|T|_{t}\right)$

Now, consider the union operation on pairs of tables $T$ and $T^{\prime}$ with the same columns that returns a table with all the tuples from the set $T \cup T^{\prime}$.

Lemma 19. For any pair of tables $T$ and $T^{\prime}$ satisfying TOA, their union table also satisfying TOA can be computed in time $O\left(\left(|T|_{o}^{2}+\left|T^{\prime}\right|_{o}^{2}\right) \times\left(|T|_{t}+\left|T^{\prime}\right|_{t}\right)\right)$.

The proofs of Lemmas 18 and 19 can be found in the appendix.

We are now in a position to describe the bottom-up query answering algorithm. Suppose we are given a program $\Pi$ in normal form. Suppose also that each extensional predicate $P$ is given by a table $T_{P}$ satisfying TOA. (This assumption can be made in all of our use-cases. Indeed, both tables TB_Sensor and Weather are naturally ordered by the timestamp, and our mappings (see Section 6) can be easily written in a way to take advantage of this order and produce tables $T$ satisfying TOA.) Thus, we can assume that the given data instance $\mathcal{D}$ is represented by a set of $T_{P}$, where each $T_{P}$ contains all the tuples $\left(c_{1}, \ldots, c_{m},\left\langle, t_{1}, t_{2},\right\rangle\right)$ such that $P\left(c_{1}, \ldots, c_{m}\right) @\left\langle t_{1}, t_{2}\right\rangle \in \mathcal{D}$. 
Consider a predicate $P$ and suppose that we have computed temporal tables $T_{P_{i}}$ satisfying TOA, for each $P_{i}$ with $P \lessdot P_{i}$ (see Section 4). We assume that the $T_{P_{i}}$ have (non-temporal) columns $\left(P_{i}, 1\right), \ldots,\left(P_{i}, m\right)$. For each rule $\alpha$ in $\Pi$ with $P$ in the head, we compute a table $T_{P}^{\alpha}$ satisfying TOA. If $\alpha$ is of the form (2), we first compute the temporal join $T$ of $T_{P_{1}}, \ldots, T_{P_{I}}$ (we change the names so that $T_{P_{i}}$ has columns $\left(P_{i}, \tau_{1}, 1\right), \ldots,\left(P_{i}, \tau_{m}, m\right)$, where $\tau_{i}=\left(\tau_{1}, \ldots, \tau_{m}\right)$, and so all the tables $T_{P_{i}}$ have distinct column names). Then we select from $T$ only those tuples $\left(c_{1}, \ldots, c_{n},\left\langle, t_{1}, t_{2},\right\rangle\right)$ for which $c_{i}=c_{j}$ in case the column names for $c_{i}$ and $c_{j}$ mention the same variable $x$, and the tuples for which $c_{i}=a$ in case the column name for $c_{i}$ mentions the constant $a$. These two steps can be done in time $O\left(\prod_{i}\left|T_{P_{i}}\right|_{o}^{2} \times \sum_{i}\left|T_{P_{i}}\right|_{t}\right)$, and the size of the resulting table does not exceed $\prod_{i}\left|T_{P_{i}}\right|_{o} \times \sum_{i}\left|T_{P_{i}}\right|_{t}$. It remains to perform projection in the following way. Suppose $P(\boldsymbol{\tau})$ with $\boldsymbol{\tau}=\left(x_{1}, \ldots x_{m}\right)$ is the head of $\alpha$ (if $\boldsymbol{\tau}$ also contains constants, the procedure below can be easily modified). Then we keep only one column among all the columns named $\left(P_{i}, x_{j}, k\right)$, for each variable $x_{j}$. It remains to rename the remaining $\left(P_{i}, x_{j}, k\right)$ to $(P, j)$, for each $j$. The total time required to compute $T_{P}^{\alpha}$ is $O\left(\prod_{i}\left|T_{P_{i}}\right|_{o}^{2} \times \sum_{i}\left|T_{P_{i}}\right|_{t}\right)$.

If $\alpha$ is of the form (4), provided that $T_{P_{1}}$ is coalesced, computing $T_{P}^{\alpha}$ reduces to using arithmetic operations for $\iota+{ }^{c} \varrho, \iota-{ }^{c} \varrho$, and $\varrho \sqsubseteq \iota$ as in the rules $\left(\boxplus_{\varrho}\right) /\left(\boxminus_{\varrho}\right)$, and projection. Therefore, $T_{P}^{\alpha}$ satisfying TOA can be computed in time $\left|T_{P_{1}}\right|_{o}^{2} \times\left|T_{P_{1}}\right|_{t}$. Computing $T_{P}^{\alpha}$ for rules of the form (3) can be done in time $O\left(\left|T_{P_{1}}\right|_{o} \times\left|T_{P_{2}}\right|_{o} \times\left(\left|T_{P_{1}}\right|_{t}+\left.T_{P_{2}}\right|_{t}\right)\right)$. Indeed, to construct $T_{P}^{\alpha}$ for a rule $\alpha$ of the form $\left.P(\boldsymbol{\tau}) \leftarrow P_{1}\left(\boldsymbol{\tau}_{1}\right) \mathcal{S}_{\varrho} P_{2}\left(\tau_{2}\right)\right)$, we follow the rule $\left(\mathcal{S}_{\varrho}\right)$ and first produce a table $T_{P_{1}}^{c}$ with the same columns as $T_{P_{1}}$, where for each tuple of $T_{P_{1}}$, we apply the operation ${ }^{c}$ to its interval. We then compute the temporal join $T$ of $T_{P_{1}}^{c}$ and $T_{P_{2}}$ after applying the renaming described above. Then we compute $T^{+^{\circ} \varrho}$ by applying the operation $+^{\circ} \varrho$ to the interval columns of each tuple in $T$, after which we compute the temporal join of $T^{+^{\circ} \varrho}$ and $T_{P_{1}}^{c}$ (with renaming applied to the columns of $T_{P_{1}}^{c}$ ). To produce $T_{P}^{\alpha}$, it remains to perform projection and renaming as described above. Finally, to compute $T_{P}$, it is sufficient to compute the union of all $T_{P}^{\alpha}$ satisfying TOA. Thus, we obtain the following, where the degree of the rule (2) is $|I|$, of (3) is 2 , and of (4) is 1 :

Lemma 20. Let $\Pi$ be a program and $P$ a predicate in it such that $K$-many rules have $P$ in the head, with $R$ being the maximal degree of those rules, $m$ the maximum of $\left|T_{P^{\prime}}\right|_{t}$ among $P^{\prime}$ such that $P \lessdot P^{\prime}$, and $n$ the maximum of $\left|T_{P^{\prime}}\right|_{o}$ among those $P^{\prime}$. Then $T_{P}$ is of size at most $n^{R} m R K$ and can be computed in time $O\left(n^{2 R} m R K\right)$.

To compute the table for the goal $Q$, we iterate the described procedure as many times as the length of the longest chain of predicates in the dependence relation $\lessdot$ for $\Pi$. Thus, we obtain:

Theorem 21. Let $m$ be the maximum of $\left|T_{P}\right|_{t}$ among the extensional predicates $P$, and $n$ the maximum of $\left|T_{P}\right|_{o}$ among those $P$. The overall time required to compute the goal predicate $Q$ of $\Pi$ is exponential in the size of $\Pi$, polynomial in $n$, and linear in $m$.

Note that if all $T_{P}$ are extracted from one table $\mathcal{R}$, as in our use-cases, then $n$ corresponds to the number of individual tuples in $\mathcal{R}$, whereas $m$ to the number of temporal intervals. It is to be emphasised that, in practice, programs tend to be small, and the number of individual constants is also small compared to the number of temporal intervals. The theorem above explains the linear patterns in our experiments below, where the size of individual tuples is fixed. 


\subsection{Implementation in SQL}

Now, we show how to rewrite a given datalog $\log _{r} M T L$ query $(\Pi, Q(\tau) @ x)$ with $\Pi$ in normal form (2)-(4) to an SQL query computing the certain answers $(\boldsymbol{c}, \iota)$ to the query with maximal intervals $\iota$. We illustrate the idea by a (relatively) simple example.

Consider the datalog $\ln _{n r} M T L$ query ( $\Pi$, HeatAffectedCounty(county)@ $x$ ), where

$$
\begin{aligned}
\Pi=\{ & \boxminus_{[0,24 h]} \text { ExcessiveHeat }(v) \leftarrow \boxminus_{[0,24 h]} \text { TempAbove24 }(v) \wedge \vartheta_{[0,24 h]} \operatorname{TempAbove41}(v), \\
& \text { HeatAffectedCounty }(v) \leftarrow \text { LocatedlnCounty }(u, v) \wedge \text { ExcessiveHeat }(u)\}
\end{aligned}
$$

is part of the meteorological ontology from Section 6. First, we transform $\Pi$ to normal form:

$$
\begin{aligned}
\Pi=\{ & \text { ExcessiveHeat }(v) \leftarrow \vartheta_{[0,24 h]} \mathrm{X}(v), \quad \mathrm{X}(v) \leftarrow \mathrm{Y}(v) \wedge \mathrm{Z}(v), \\
& \mathrm{Y}(v) \leftarrow \boxminus_{[0,24 h]} \operatorname{TempAbove24}(v), \mathrm{Z}(v) \leftarrow \vartheta_{[0,24 h]} \text { TempAbove41 }(v), \\
& \text { HeatAffectedCounty }(v) \leftarrow \text { LocatedInCounty }(u, v) \wedge \text { ExcessiveHeat }(u)\} .
\end{aligned}
$$

We regard TempAbove24, TempAbove41, LocatedlnCounty as extensional predicates given by the tables $T_{\text {TempAbove24 }}, T_{\text {TempAbove41 }}, T_{\text {LocatedlnCounty. The first two of these tables have columns }}$ station_id, ledge, redge, and the third one station_id, county, ledge, redge. To simplify presentation, we omit the columns Ipar and rpar used in the previous section and assume that all the temporal intervals take the form $\left(t, t^{\prime}\right]$; see Section 6.

For each predicate $P$ in $\Pi$, we also create a view (temporary table) $V_{P}^{*}$ with the same columns as $T_{P}$. We set $V_{P}^{*}=$ coalesce $\left(T_{P}\right)$, where coalesce is a query that implements coalescing in $\mathrm{SQL}^{3}$ We explain the idea behind this query for a temporal table $T$ (as mentioned above, we omit columns lpar, rpar). For a moment of time $t$ occurring in $T$, we denote by $b^{\geq}(T, t)$ the number of $i$ such that $T[i$, ledge $] \geq t$, and by $e^{\geq}(T, t)$ the number of $i$ such that $T[i$, redge $] \geq t$; the numbers $b \leq(T, t)$ and $e^{\leq}(T, t)$ are defined analogously. It can be readily seen that every $t$ in $T$ [ledge] such that $b^{\geq}(T, t)=e^{\geq}(T, t)$ is the beginning of some interval in the coalesced table $T^{*}$. Similarly, every $t^{\prime}$ in $T$ [redge] such that $b \leq\left(T, t^{\prime}\right)=e^{\leq}\left(T, t^{\prime}\right)$ is the end of some interval in $T^{*}$. The coalesced intervals of $T^{*}$ can be then obtained as pairs $\left(t, t^{\prime \prime}\right]$, where $t$ is as above and $t^{\prime \prime}$ is the minimum over those $t^{\prime}$ defined above that are $\geq t$. Thus, to coalesce $T_{\text {TempAbove24 }}$ we first use the query

$$
\begin{aligned}
& V_{l}=\text { SELECT } T \text {.station_id AS station_id, } T \text {.ledge AS ledge FROM } T_{\text {TempAbove24 }} T \text { WHERE } \\
& \text { (SELECT COUNT }(*) \text { from } T \text { TempAbove24 } S \text { WHERE } S \text {.ledge } \geq T \text {.ledge AND } \\
& S . \text { station_id }=T \text {.station_id) }= \\
& \text { (SELECT COUNT }(*) \text { from } T_{\text {TempAbove24 }} S \text { WHERE } S \text {.redge } \geq T \text {.ledge AND } \\
& \text { S.station_id }=T \text {.station_id), }
\end{aligned}
$$

which extracts the pairs $(n, t)$, where $t$ is as described above and station_id $=n$. An analogous query can be used to produce $V_{r}$, a table of pairs $\left(n, t^{\prime}\right)$, where $t^{\prime}$ is as described above and

3. It should not be confused with the standard coalesce function in SQL that returns the first of its arguments that is not null, or null if all of the arguments are null. 
station_id $=n$. Finally, we set

$$
\begin{aligned}
& V_{\text {TempAbove24 }}^{*}=\operatorname{SELECT} V_{l} \text {.station_id AS station_id, } V_{l} \text {.ledge AS ledge, } \\
& \left(\begin{array}{c}
\text { SELECT MIN }\left(V_{r} \text {.redge }\right) \text { FROM } V_{r} \text { WHERE } V_{r} \text {.redge } \geq V_{l} \text {.ledge AND } \\
\left.V_{l} \text {.station_id }=V_{r} \text {.station_id }\right) \text { AS redge }
\end{array}\right.
\end{aligned}
$$

FROM $V_{l}$.

A more efficient variant of this algorithm that uses window functions with sorting and partitioning allows us to avoid joins used, e.g., in the query $V_{l}$ (Zhou, Wang, \& Zaniolo, 2006). We will refer to this algorithm in Section 7 as a standard $S Q L$ algorithm. In contrast to the imperative algorithm described in the proof of Lemma 16, this algorithm can be implemented using standard SQL operators.

In addition, for each intensional predicate $P$ of $\Pi$, we create a view $V_{P}$ defined by an SQL query that reflects the definitions of $P$ in $\Pi$. For example, we set

$$
\begin{aligned}
V_{\mathrm{Y}}= & \text { SELECT } V_{\text {TempAbove24.station_id AS station_id, }}^{*} \\
& V_{\text {TempAbove24. }}^{*} \text { ledge }+24 h \text { AS ledge, } V_{\text {TempAbove24.redge AS redge, }}^{*} \text {. } \\
& \text { FROM } V_{\text {TempAbove24 }}^{*} \text { WHERE } V_{\text {TempAbove24 }}^{*} \cdot \text { redge }-V_{\text {TempAbove24. }}^{*} \text { ledge } \geq 24 h .
\end{aligned}
$$

This query implements the $\iota+{ }^{c} \varrho$ operation for $\varrho=[0,24 h]$, and the WHERE clause checks whether $\varrho \sqsubseteq \iota$ holds, where $\iota=\left(V_{\text {TempAbove24 }}^{*}\right.$.ledge, $V_{\text {TempAbove24 }}^{*}$.redge $]$. We then set $V_{Y}^{*}=\operatorname{coalesce}\left(V_{Y}\right)$ and note that the query

$$
\text { SELECT station_id, ledge, redge FROM } V_{Y}^{*} \text {, }
$$

when evaluated over the tables $T_{\text {TempAbove24 }}, T_{\text {TempAbove41 }}$ and $T_{\text {LocatedlnCounty }}$, would produce the answers to the query $\left(\Pi, \mathrm{Y}\right.$ (station_id, county)@x) with maximal intervals $\iota=\left(\iota_{b}, \iota_{e}\right]$, where $\iota_{b}$ corresponds to ledge, and $\iota_{e}$ to redge.

We now explain how to construct queries for the concepts whose definitions involve $\wedge$ using the example of HeatAffectedCounty:

$$
\begin{aligned}
& V_{\text {HeatAffectedCounty }}=\operatorname{SELECT} V_{\text {LocatedInCounty }}^{*} \cdot \text { county AS county, } \\
& \qquad \begin{array}{r}
\operatorname{MX}\left(V_{\text {LocatedInCounty }}^{*} \cdot \text { ledge, } V_{\text {ExcessiveHeat }}^{*} \cdot \text { ledge }\right) \text { AS ledge, } \\
\operatorname{MN}\left(V_{\text {LocatedInCounty }}^{*} \cdot \text { redge }, V_{\text {ExcessiveHeat }}^{*} \cdot \text { redge }\right) \text { AS redge } \\
\text { FROM } V_{\text {LocatedInCounty }}^{*}, V_{\text {ExcessiveHeat }}^{*}
\end{array}
\end{aligned}
$$

$$
\begin{aligned}
& \text { WHERE } \operatorname{MX}\left(V_{\text {LocatedInCounty }}^{*} \cdot \text { ledge }, V_{\text {ExcessiveHeat }}^{*} \cdot \text { ledge }\right)< \\
& \operatorname{MN}\left(V_{\text {LocatedInCounty }}^{*} \cdot \text { redge }, V_{\text {ExcessiveHeat }}^{*} \cdot \text { redge }\right) \\
& \text { AND } V_{\text {LocatedInCounty }}^{*} \cdot \text { county }=V_{\text {ExcessiveHeat }}^{*} \cdot \text { county },
\end{aligned}
$$

where MN (MX) is the function that returns the earliest (latest) of any two given date/time values (it can be implemented in SQL as a user-defined function, or using the CASE operator). Finally, we use a query similar to (24) over $V_{\text {HeatAffectedCounty }}^{*}$ to produce the answers to $(\Pi, \boldsymbol{q}($ county, $x)$ ). 


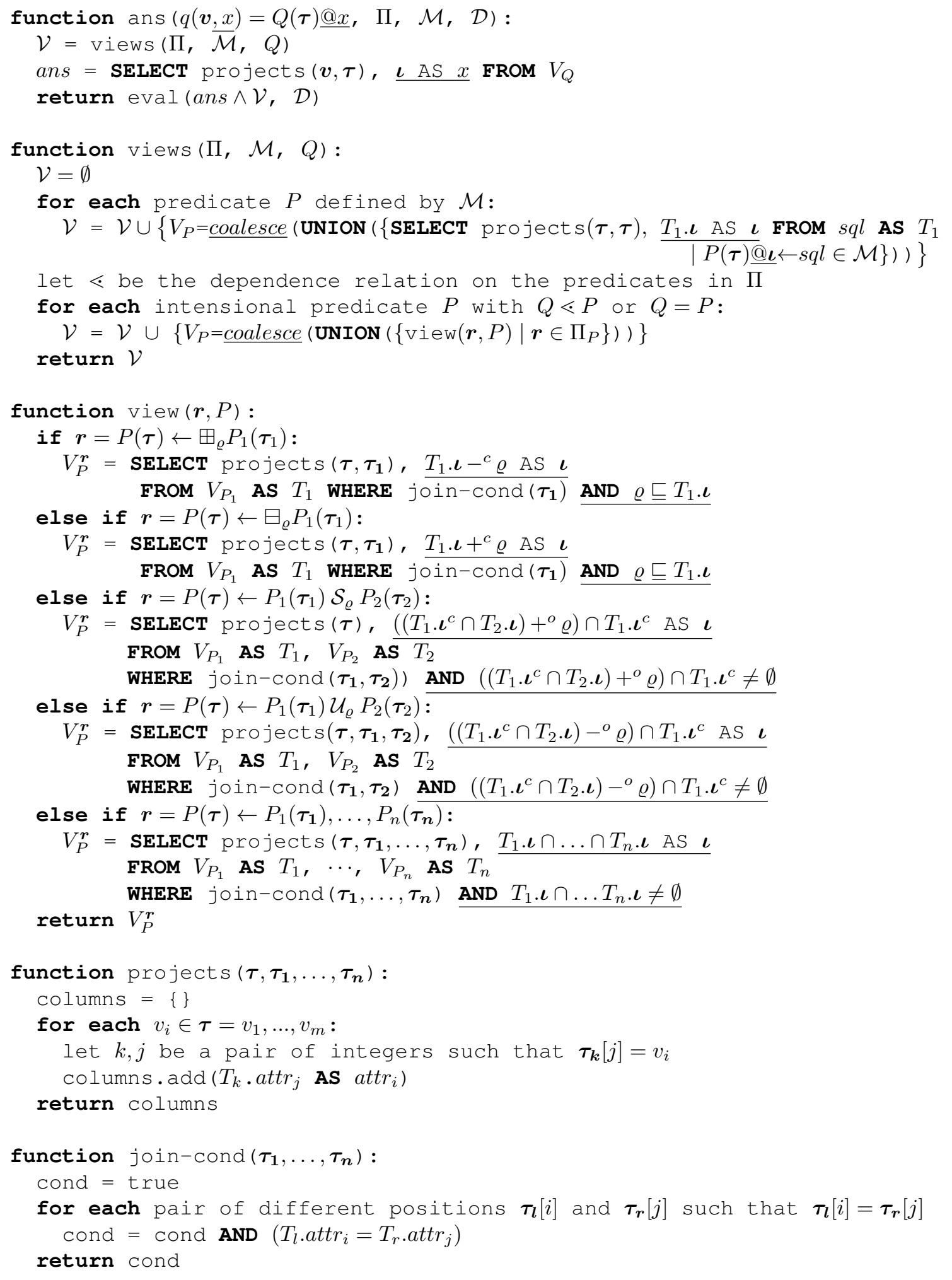

Figure 3: The algorithm for evaluating data $\log _{n r} M T L$ queries in SQL. 
We are mostly interested in the scenario where the tables $T_{P}$ are not available immediately, but extracted from raw timestamped data tables $R$ by means of mappings. In this case, we use views $V_{P}$ instead of $T_{P}$ defined over $R$. For example, if the raw data is stored in the table Weather, we define the view:

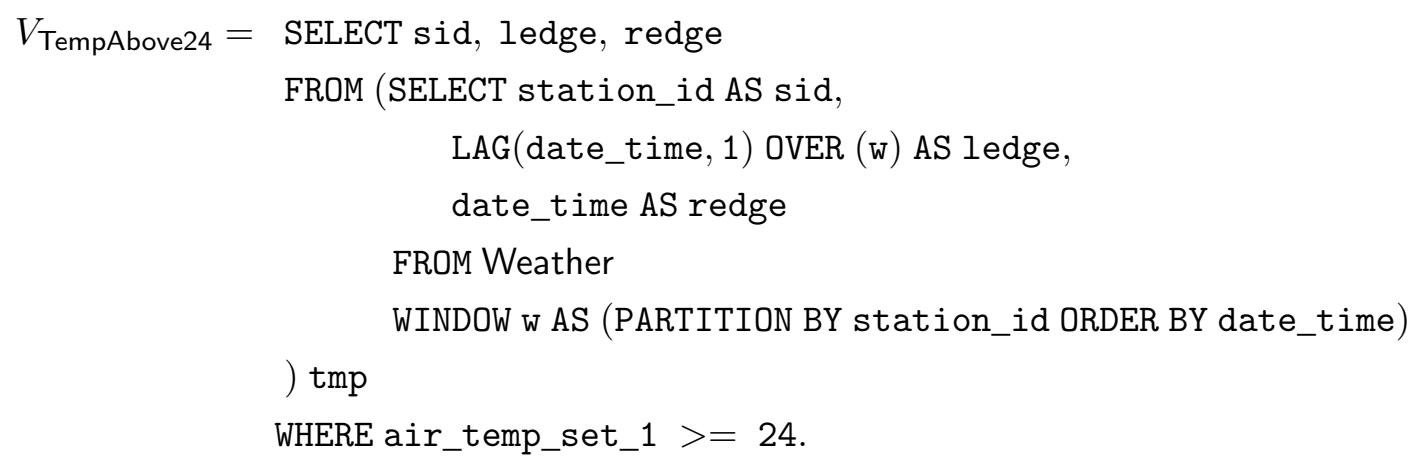

Our general rewriting algorithm is outlined in Fig. 3, where the function ans produces an SQL query that computes the certain answers to $(\Pi, Q(\tau) @ x)$ (with maximal intervals) by evaluating the query over the input database $\mathcal{D}$. The algorithm is a variation of the standard translation of nonrecursive Datalog to relational algebra—see, e.g., the work by Ullman (1988)—extended with the operations on temporal intervals described above (they are underlined in Fig. 3).

It is to be noted that the 'views' introduced by the algorithm do not require modifying the underlying database. They can be implemented in different ways: for example, by using subqueries, common table expressions (CTEs), or temporary tables. For the experiments in Section 7, we use the last approach, where temporary tables are generated on the fly and exist only within a transaction.

\section{Use Cases}

We test the feasibility of OBDA with datalog $\ln _{n r} M T L$ by querying Siemens turbine log data and MesoWest weather data. In this section, we briefly describe these use cases; detailed results of our experiments will presented in Section 7.

\subsection{Siemens}

Siemens service centres store aggregated turbine sensor data in tables such as TB_Sensor. The data comes with (not necessarily regular) timestamps $t_{1}, t_{2}, \ldots$, and it is deemed that the values remain constant in every interval $\left[t_{i}, t_{i+1}\right)$. Using a set of mappings, we extract from these tables a data instance containing ground facts such as

$$
\begin{aligned}
& \text { ActivePowerAbove1.5(tb0)@[12:20:48, 12:20:49), } \\
& \text { ActivePowerAbove1.5(tb0)@[12:20:49, 12:20:52), } \\
& \text { RotorSpeedAbove1500(tb0)@[12:20:48, 12:20:49), } \\
& \text { MainFlameBelow0.1(tb0)@[12:20:48, 12:20:52). }
\end{aligned}
$$




\subsection{MesoWest}

The MesoWest (http://mesowest.utah.edu/) project makes publicly available historical records of the weather stations across the US showing such parameters of meteorological conditions as temperature, wind speed and direction, amount of precipitation, etc. Each station outputs its measurements with some periodicity, with the output at time $t_{i+1}$ containing the accumulative (e.g., for precipitation) or averaged (e.g., for wind speed) value over the interval $\left(t_{i}, t_{i+1}\right]$. The data comes in a table Weather, which looks as follows:

\begin{tabular}{|c|c|c|c|c|c|c|}
\hline stationId & dateTime & airTemp & windSpeed & windDir & hourPrecip & $\ldots$ \\
\hline & & $\ldots$ & & & & \\
KBVY & $2013-02-15 ; 15: 14$ & 8 & 45 & 10 & 0.05 & \\
KMNI & $2013-02-15 ; 15: 21$ & 6 & 123 & 240 & 0 & \\
KBVY & $2013-02-15 ; 15: 24$ & 8 & 47 & 10 & 0.08 & \\
KMNI & $2013-02-15 ; 15: 31$ & 6.7 & 119 & 220 & 0 & \\
& & $\ldots$ & & & & \\
\hline
\end{tabular}

One more table, Metadata, provides some atemporal meta information about the stations:

\begin{tabular}{|c|c|c|c|c|c|}
\hline stationId & county & state & latitude & longitude & $\ldots$ \\
\hline & & $\ldots$ & & & \\
KBVY & Essex & Massachusetts & 42.58361 & -70.91639 & \\
KMNI & Essex & Massachusetts & 33.58333 & -80.21667 & \\
& & $\ldots$ & & & \\
\hline
\end{tabular}

The monitoring and historical analysis of the weather involves answering queries such as "find showery counties, where one station observes precipitation at the moment, while another one does not, but observed precipitation 30 minutes ago'.

We use SQL mappings over the Weather table similar to those in the Siemens case to obtain ground atoms such as

$$
\begin{aligned}
& \text { NorthWind(KBVY)@(15:14, 15:24], } \\
& \text { HurricaneForceWind(KMNI)@(15:21, 15:31], } \\
& \text { Precipitation(KBVY)@(15:14, 15:24], } \\
& \text { TempAbove0(KBVY)@(15:14, 15:24], } \\
& \text { TempAbove0(KMNI)@(15:21, 15:31] }
\end{aligned}
$$

(according to the standard definition, the hurricane force wind is above $118 \mathrm{~km} / \mathrm{h}$ ). On the other hand, mappings to the Metadata table provide atoms such as

$$
\begin{aligned}
& \text { LocatedlnCounty(KBVY, Essex }) @(-\infty, \infty), \\
& \text { LocatedInState(KBVY, Massachusetts)@ }(-\infty, \infty) .
\end{aligned}
$$

Our ontology contains definitions of various meteorological terms:

$$
\begin{aligned}
& \text { ShoweryCounty }(v) \leftarrow \text { LocatedlnCounty }\left(u_{1}, v\right) \wedge \text { LocatedlnCounty }\left(u_{2}, v\right) \wedge \\
& \text { Precipitation }\left(u_{1}\right) \wedge \operatorname{NoPrecipitation}\left(u_{2}\right) \wedge \vartheta_{(0,30 m]} \operatorname{Precipitation}\left(u_{2}\right), \\
& \Xi_{[0,1 h]} \text { Hurricane }(v) \leftarrow \boxminus_{[0,1 h]} \operatorname{HurricaneForceWind}(v), \\
& \text { HurricaneAffectedState }(v) \leftarrow \text { LocatedInState }(u, v) \wedge \operatorname{Hurricane}(u),
\end{aligned}
$$




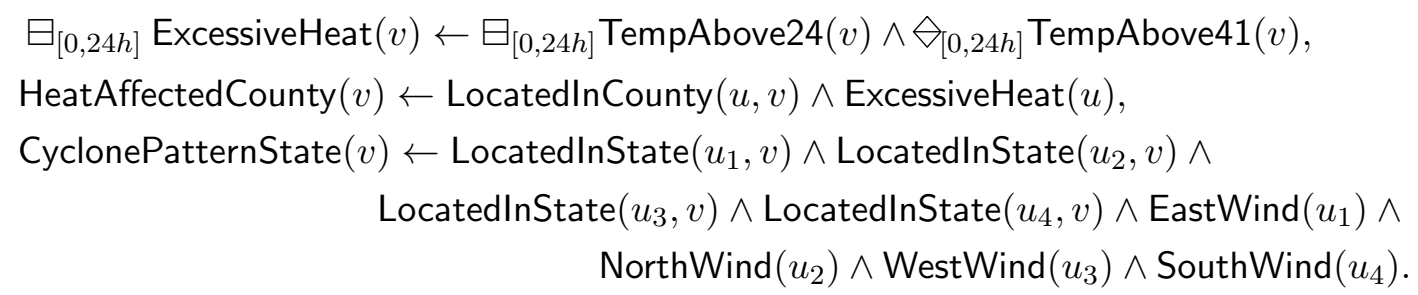

\section{Experiments}

To evaluate the performance of the SQL queries produced by the datalog $\ln _{n r} M T L$ rewriting algorithm outlined in Section 5.2, we developed two benchmarks for our use cases. We ran the experiments on an HP Proliant server with 2 Intel Xeon X5690 Processors (with 12 logical cores at 3.47GHz each), 106GB of RAM and five 1TB 15K RPM HD. We used both PostgreSQL 9.6 and the SQL interface (Armbrust, Xin, Lian, Huai, Liu, Bradley, Meng, Kaftan, Franklin, Ghodsi, \& Zaharia, 2015) of Apache Spark 2.1.0. Apache Spark is a cluster-computing framework that provides distributed task dispatching, scheduling and data parallelisation. For each of these two systems, we provided two different implementations, imperative and standard SQL, which diverge in the computation of maximal intervals; see Section 5. We ran all the queries with a timeout of 30 minutes.

\subsection{Siemens}

Siemens provided us with a sample of data for one running turbine, which we denote by tb0, over 4 days in the form of the table TB_Sensor. The data table was rather sparse, containing a lot of nulls, because different sensors recorded data at different frequencies. For example, ActivePower arrived most frequently with average periodicity of 7 seconds, whereas the values for the field MainFlame arrived most rarely, every 1 minute on average. We replicated this sample to imitate the data for one turbine over 10 different periods ranging from 32 to 320 months. The statistics of the data sets are given in Tables 4 and 13. We evaluated four queries ActivePowerTrip(tb0)@x, NormalStart(tb0)@x, NormalStop(tb0)@x and NormalRestart(tb0)@x. The statistics of returned answers is given in Table 10.

\begin{tabular}{|c|c|c|c|c|c|c|c|c|c|c|}
\hline \# of months & 32 & 64 & 96 & 128 & 159 & 191 & 223 & 255 & 287 & 320 \\
\hline \# of rows (approx.) & $13 \mathrm{M}$ & $26 \mathrm{M}$ & $39 \mathrm{M}$ & $52 \mathrm{M}$ & $65 \mathrm{M}$ & $77 \mathrm{M}$ & $90 \mathrm{M}$ & $103 \mathrm{M}$ & $116 \mathrm{M}$ & $129 \mathrm{M}$ \\
\hline size (GB) in CSV & 0.57 & 1.2 & 1.7 & 2.3 & 2.9 & 3.4 & 4.0 & 4.5 & 5.1 & 5.7 \\
\hline
\end{tabular}

Table 4: Siemens data for one turbine.

The execution times for the Siemens use case are given in Fig. 5. Although Apache Spark was designed to perform efficient parallel computations, it failed to take advantage of this feature due to the fact that the Siemens data could not be partitioned by mapping each part to a separate core. PostgreSQL 9.6 also supports parallel query execution in some cases. However, as many operators (e.g., scans of temporary tables) in our queries are classified either 'parallel unsafe' or 'parallel restricted' in the parallel safety documentation (PostgreSQL, 2018), the query planner failed to produce any parallel execution strategy in our case. The reason why PostgreSQL outperformed Apache Spark is that the latter does not provide a convenient way to define proper indexes over temporary tables, which leads to quadratically growing running times. On the other hand, PostgreSQL shows linear growth in the size of data (confirming theoretical results since we deal with a single turbine). 

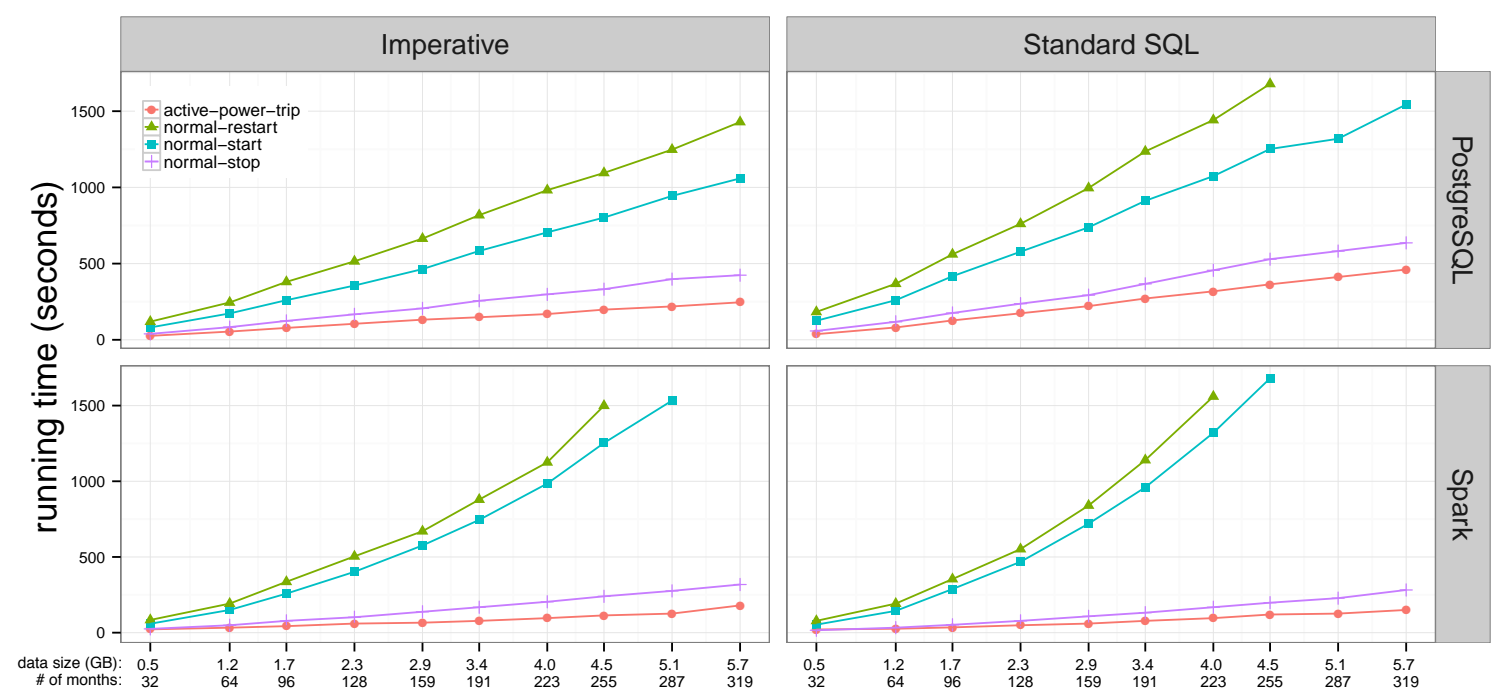

Figure 5: Experiment results for the Siemens use case.

\begin{tabular}{|c|c|c|c|c|c|c|c|c|c|c|}
\hline $\begin{array}{c}\text { \# of years } \\
\text { \# of stations }\end{array}$ & 1 & 2 & 3 & 4 & 5 & 6 & 7 & 8 & 9 & 10 \\
\hline \# of rows (approx.) & $4 \mathrm{M}$ & 306 & 370 & 441 & 484 & 542 & 595 & 643 & 807 & 874 \\
\hline size (GB) in CSV & 0.2 & 0.6 & $19 \mathrm{M}$ & $27 \mathrm{M}$ & $36 \mathrm{M}$ & $49 \mathrm{M}$ & $63 \mathrm{M}$ & $79 \mathrm{M}$ & $99 \mathrm{M}$ & $124 \mathrm{M}$ \\
\hline
\end{tabular}

Table 6: NY weather stations from 2005 to 2014.

Note that the normal restart (start) query timeouts on the data for more than 18 (respectively, 21) years, which is more than enough for the monitoring and diagnostics tasks at Siemens, where the two most common application scenarios for sensor data analytics are daily monitoring (that is, analytics of high-frequency data of the previous 24 hours) and fleet-level analytics of key-performance indicators over one year. In both cases, the computation time of the results is far less a crucial cost factor than the lead-time for data preparation.

\subsection{MesoWest}

In contrast to the Siemens case, the weather tables contain very few nulls. Normally, the data values arrive with periodicity from 1 to 20 minutes. We tested the performance of our algorithm by increasing $(i)$ the temporal span (with some necessary increase of the spatial spread) and $(i i)$ the geographical spread of data. For $(i)$, we took the New York state data for the 10 continuous periods between 2005 and 2014; see Tables 6 and 14. As each year around 70 new weather stations were added, our 10 data samples increase more than linearly in size. For $(i i)$, we fixed the time period of one year (2012) and linearly increased the data from 1 to 19 states (NY, NJ, MD, DE, GA, RI, MA, CT, LA, VT, ME, WV, NH, NC, MS, SC, ND, KY, SD); see Table 7 and 15. In both cases, we executed four datalog $\ln _{n} M T L$ queries ShoweryCounty $(v) @ x$, HurricaneAffectedState $(\mathrm{NY}) @ x$, HeatAffectedCounty $(v) @ x$, CyclonePatternState(NY)@ $x$. The statistics of the returned answers is shown in Tables 11 and 12. 

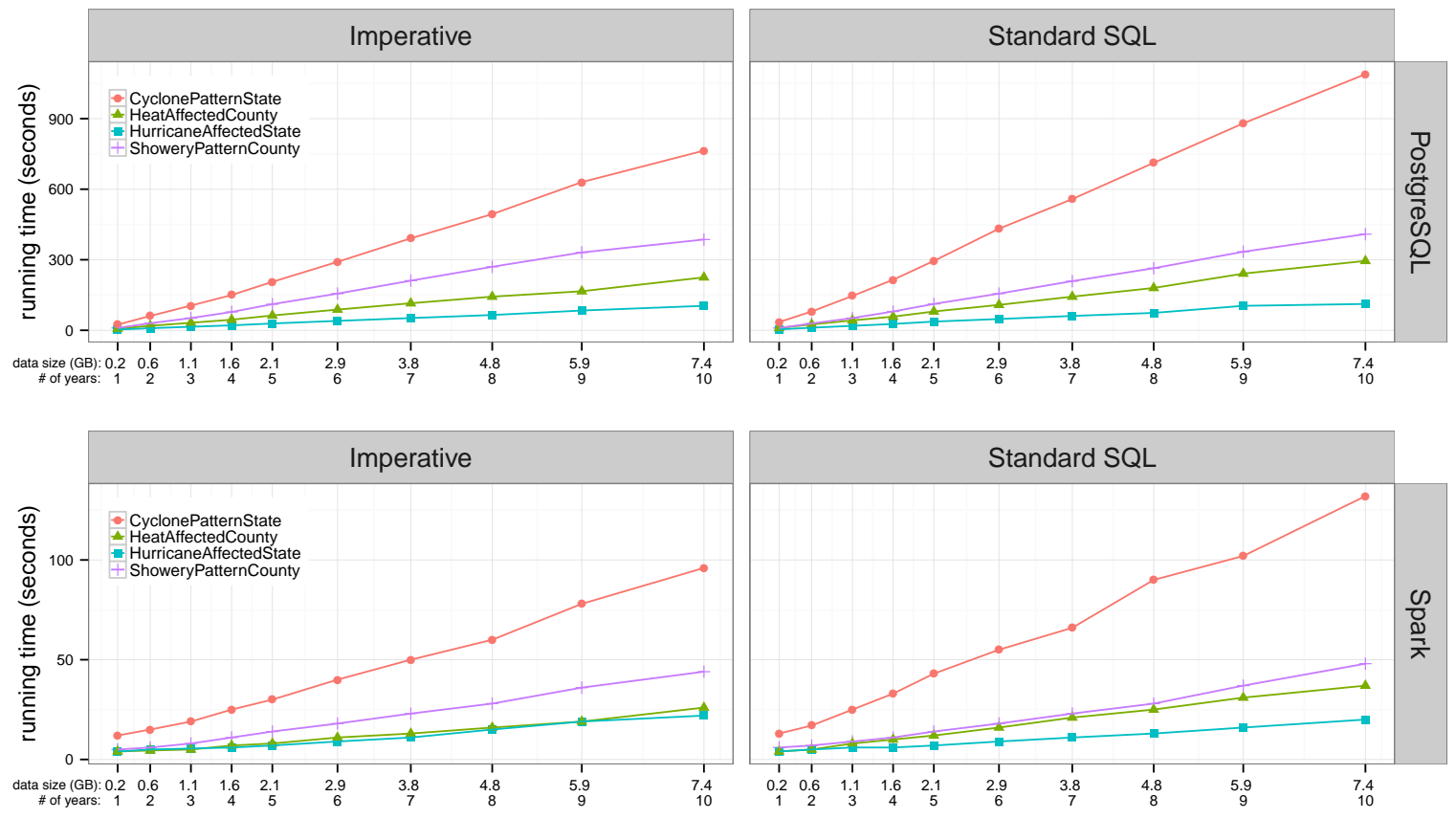

Figure 8: Experiments over New York data of 2005-2014 with PostgreSQL and Spark
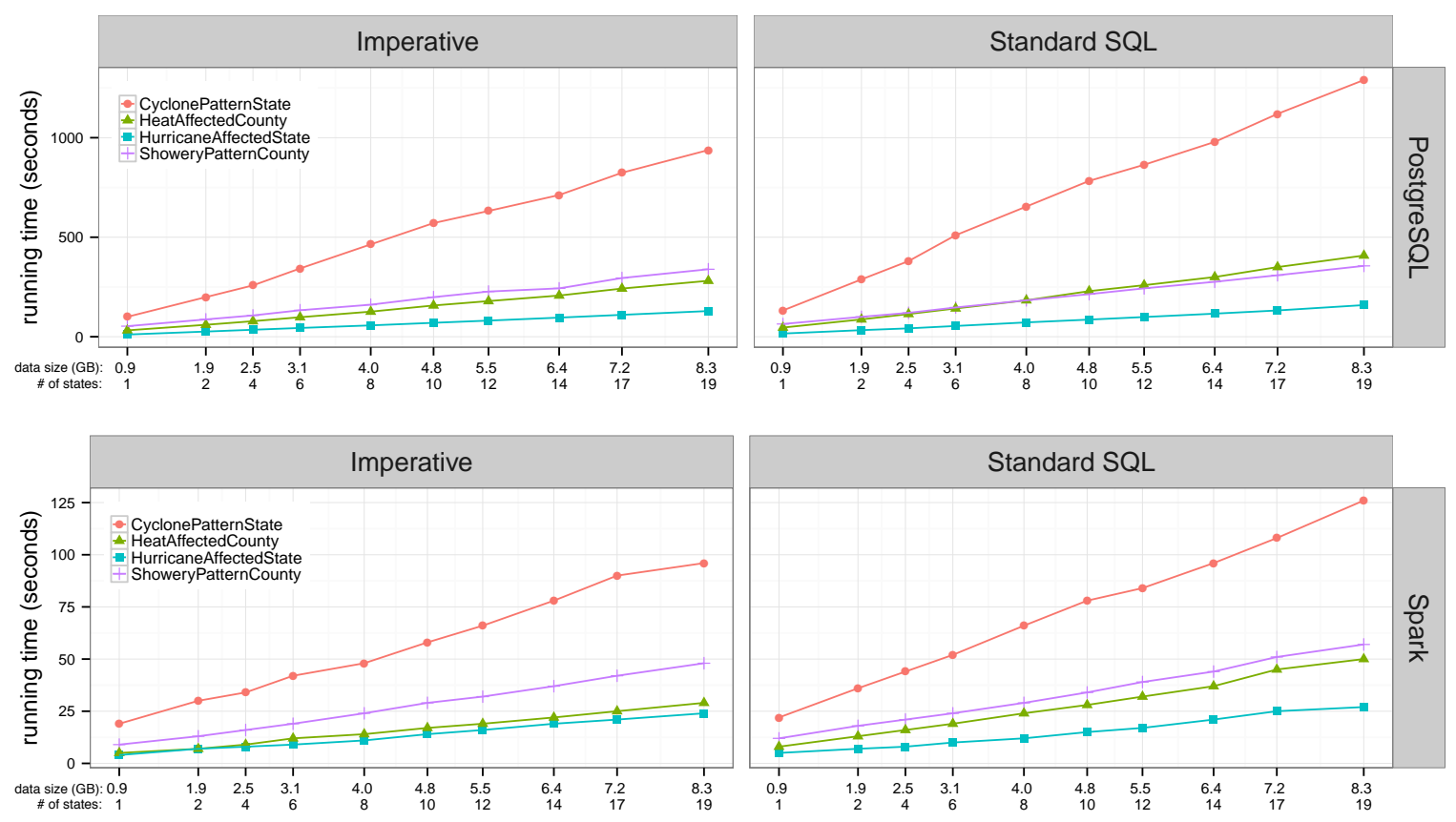

Figure 9: Experiments over 1 year data from 1-19 states with PostgreSQL and Spark. 


\begin{tabular}{|c|c|c|c|c|c|c|c|c|c|c|}
\hline states & $\begin{array}{c}\text { DE, } \\
\text { GA }\end{array}$ & $+\mathrm{NY}$ & $+\mathrm{MD}$ & $\begin{array}{c}+\mathrm{NJ}, \\
\mathrm{RI}\end{array}$ & $\begin{array}{c}+\mathrm{MA}, \\
\mathrm{CT}\end{array}$ & $\begin{array}{c}+\mathrm{LA}, \\
\mathrm{VT}\end{array}$ & $\begin{array}{c}+\mathrm{ME}, \\
\mathrm{WV}\end{array}$ & $\begin{array}{c}+\mathrm{NH}, \\
\text { NC }\end{array}$ & $\begin{array}{c}+\mathrm{MS}, \mathrm{SC}, \\
\text { ND }\end{array}$ & $\begin{array}{c}+\mathrm{KY}, \\
\text { SD }\end{array}$ \\
\hline \# of states & 2 & 3 & 4 & 6 & 8 & 10 & 12 & 14 & 17 & 19 \\
\# of stations & 408 & 659 & 1120 & 1476 & 1875 & 2305 & 2669 & 3019 & 3508 & 4037 \\
\hline \# of rows (approx.) & $17 \mathrm{M}$ & $32 \mathrm{M}$ & $41 \mathrm{M}$ & $52 \mathrm{M}$ & $67 \mathrm{M}$ & $81 \mathrm{M}$ & $93 \mathrm{M}$ & $106 \mathrm{M}$ & $121 \mathrm{M}$ & $141 \mathrm{M}$ \\
\hline size (GB) in CSV & 0.9 & 1.9 & 2.5 & 3.1 & 4.0 & 4.8 & 5.5 & 6.4 & 7.2 & 8.3 \\
\hline
\end{tabular}

Table 7: Weather data for 1-19 states in 2012.

The execution times are shown in Figures 8 and 9. All the four queries can be answered within the time limit. The most expensive one is the cyclone pattern state query because its definition includes a join of four atoms for winds in four directions, each with a large volume of instances. All the graphs in Figures 9 and 8 exhibit linear behaviour with respect to the size of data. The nearly tenfold better performance of Spark over PostgreSQL can be explained by the fact that, unlike the data in the Siemens case, the MesoWest data is highly parallelisable. Since it was collected from hundreds of different weather stations, it can be partitioned by station id, state, county, etc. to perfectly fit the MapReduce programming model extended with resilient distributed datasets (RDDs) (Zaharia, Xin, Wendell, Das, Armbrust, Dave, Meng, Rosen, Venkataraman, Franklin, Ghodsi, Gonzalez, Shenker, $\&$ Stoica, 2016). In this case, Apache Spark is able to take advantage of the multi-core and large memory hardware infrastructure, to compute mappings and coalescing in parallel, making it 10 times faster than PostgreSQL; see Figures 8 and 9.

Overall, the results of the experiments look very encouraging: our datalog ${ }_{n r} M T L$ query rewriting algorithm produces SQL queries that are executable by a standard database engine PostgreSQL in acceptable time, and by a cluster-computing framework Apache Spark in better than acceptable time (in case data can be properly partitioned) over large sets of real-world temporal data of up to $8.3 \mathrm{~GB}$ in CSV format. The relatively challenging queries such as NormalRestart and CyclonePatternState require a large number of temporal joins, which turn out to be rather expensive.

\section{Conclusions and Future Work}

To facilitate access to sensor temporal data with the aim of monitoring and diagnostics, we suggested the ontology language datalogMTL, an extension of datalog with the Horn fragment of the metric temporal logic MTL (under the continuous semantics). We showed that answering datalogMTL queries is EXPSPACE-complete for combined complexity, but becomes undecidable if the diamond operators are allowed in the head of rules. We also proved that answering nonrecursive datalogMTL queries is PSPACE-complete for combined complexity and in $\mathrm{AC}^{0}$ for data complexity. We tested feasibility and efficiency of OBDA with datalog ${ }_{n r} M T L$ on two real-world use cases by querying Siemens turbine data and MesoWest weather data. Namely, we designed data $\log _{n r} M T L$ ontologies defining typical concepts used by Siemens engineers and various meteorological terms, developed and implemented an algorithm rewriting datalog $\log _{n r} M T L$ queries into SQL queries, and then executed the SQL queries obtained by this algorithm from our ontologies over the Siemens and MesoWest data, showing their acceptable efficiency and scalability. (To the best of our knowledge, this is the first work on practical OBDA with temporal ontologies, and so no other systems with similar functionalities are available for comparison.) 
Based on these encouraging results, we plan to include our temporal OBDA framework into the Ontop platform (Rodriguez-Muro et al., 2013; Kontchakov, Rezk, Rodriguez-Muro, Xiao, \& Zakharyaschev, 2014; Calvanese et al., 2017); visit http: / / ontop. inf . unibz. it/ for more information on Ontop. Note also that datalogMTL presented here has been recently used to develop an ontology of ballet moves (see Example 2) that underlies a search engine of annotated sequences in ballet videos (Raheb et al., 2017). This is a third use case for our framework (and we are aware of a few more emerging use cases), which makes an efficient and user-friendly implementation of the framework a top priority.

We are also working on the streaming data setting, where the challenge is to continuously evaluate queries over the incoming data. A rule-based language with window operators for analysing streaming data has been suggested by Beck, Dao-Tran, Eiter, and Fink (2015). This language is very expressive as it uses an abstract semantics for window operators (which does not have to guarantee decidability) and allows negation and disjunction in the rules. It would be interesting to identify and adapt a suitable fragment of this language in our temporal OBDA framework.

\section{Acknowledgements}

This work was supported by the UK EPSRC grant EP/M012670 'iTract: Islands of Tractability in Ontology-Based Data Access' and by the OBATS project at the Free University of Bozen-Bolzano.

Guohui Xiao is the corresponding author of this article.

\section{Appendix A.}

\section{Proof of Theorem 14}

The formula $\sigma_{\varrho, P, P_{1}, P_{2}}^{\langle m, n\rangle}(x, y)$ is defined as follows:

$$
\begin{aligned}
& \exists x_{1}, y_{1}, \ldots, x_{5}, y_{5} \bigvee_{\substack{m_{1} \in \operatorname{le}\left(P_{1}\right) \\
n_{1} \in \operatorname{ri}\left(P_{1}\right) \\
\left\lceil_{1} \in\left\{[,(\},\rceil_{1} \in\{],\right)\right\}}}\left(\varphi _ { P _ { 1 } } ^ { \lceil _ { 1 } m _ { 1 } , n _ { 1 } \rceil _ { 1 } } ( x _ { 1 } , y _ { 1 } ) \wedge \bigvee _ { \substack { m _ { 2 } \in \operatorname { l e } ( P _ { 2 } ) \\
n _ { 2 } \in \operatorname { r i } ( P _ { 2 } ) \\
\lceil _ { 2 } \in \{ [ , ( \} , \rceil _ { 2 } \in \{ ] , ) \} } } \left(\varphi_{P_{2}}^{\left\lceil_{2} m_{2}, n_{2}\right]_{2}}\left(x_{2}, y_{2}\right) \wedge\right.\right. \\
& \bigvee_{\substack{m_{3}=m_{1} \\
n_{3}=n_{1}}}\left(\left(x_{3}=x_{1}\right) \wedge\left(y_{3}=y_{1}\right) \wedge \text { is }_{\left\lceil_{3},[\right.} \wedge \text { is }_{\rceil_{3},\right]} \wedge\right. \\
& \left\lceil_{3} \in\left\{[,(\},\rceil_{3} \in\{],\right)\right\} \\
& \bigvee_{\substack{\left.m_{4} \in \operatorname{le}\left(P_{1}\right) \cup \operatorname{le}\left(P_{2}\right) \\
n_{4} \in \operatorname{ri}\left(P_{1}\right) \cup \operatorname{ri}\left(P_{2}\right) \\
\Gamma_{4} \in\left\{[,(\},\rceil_{4} \in\{],\right)\right\}}}\left(\operatorname{inter}_{\left\lceil_{2} m_{2}, n_{2}\right\rceil_{2},\left\lceil_{3} m_{3}, n_{3}\right\rceil_{3}}^{\left\lceil_{4}, n_{4}\right\rceil_{4}}\left(x_{4}, y_{4}, x_{2}, y_{2}, x_{3}, y_{3}\right) \wedge\right. \\
& \bigvee_{\substack{\left.m_{4} \in \operatorname{le}(P) \\
n_{4} \in \operatorname{ri}(P) \\
\Gamma_{5} \in\left\{[,(\},\rceil_{5} \in\{],\right)\right\}}}\left(\text { pluso }_{\left.\varrho, \Gamma_{4} m_{4}, n_{4}\right\rceil_{4}}^{\left.\Gamma_{5} m_{5}, n_{5}\right\rceil_{5}}\left(x_{5}, y_{5}, x_{4}, y_{4}\right) \wedge\right. \\
& \left.\left.\left.\left.\operatorname{inter}\left\langle_{\left\lceil_{5} m_{5}, n_{5}\right\rceil_{5},\left\lceil_{3} m_{3}, n_{3}\right\rceil_{3}}^{\langle m, n\rangle}\left(x, y, x_{5}, y_{5}, x_{3}, y_{3}\right)\right)\right)\right)\right)\right) \text {, }
\end{aligned}
$$

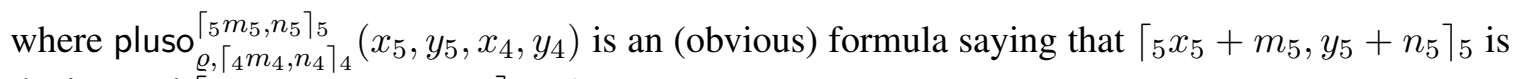
the interval $\left\lceil_{4} x_{4}+m_{4}, y_{4}+n_{4}\right\rceil_{4}+{ }^{o} \varrho$. 
The formula $x=y+c$, for a non-negative $c$, is defined as follows. For $c=\infty$, we take the formula

$$
\forall j\left(\operatorname{bit}^{i n}(x, j, 1) \wedge \operatorname{bit}^{f^{r}}(x, j, 1)\right),
$$

whereas for a constant $c=h / 2^{k}$, we can use

$$
\begin{aligned}
\forall j\left(\left(\operatorname{bit}^{i n}(x, j, 0)\right.\right. & \left.\left.\wedge \operatorname{bit}_{+h / 2^{k}}^{i n}(y, j, 0)\right) \vee\left(\operatorname{bit}^{i n}(x, j, 1) \wedge \mathrm{bit}_{+h / 2^{k}}^{i n}(y, j, 1)\right)\right) \wedge \\
\forall j\left(\left(\operatorname{bit}^{f r}(x, j, 0) \wedge \mathrm{bit}_{+h / 2^{k}}^{f r}(y, j, 0)\right)\right. & \left.\vee\left(\operatorname{bit}^{f r}(x, j, 1) \wedge \mathrm{bit}_{+h / 2^{k}}^{f r}(y, j, 1)\right)\right),
\end{aligned}
$$

where predicates $\operatorname{bit}_{+h / 2^{k}}^{i n}(y, j, v)$, saying that $v$ is the $j$-th bit of the integer part of $y+h / 2^{k}$, and $\mathrm{bit}_{+h / 2^{k}}^{f r}(y, j, v)$, saying that $v$ is the $j$-th bit of the fractional part of $y+h / 2^{k}$, are defined inductively as follows:

$$
\begin{aligned}
& \mathrm{bit}_{+0 / 2^{k}}^{f_{r}}(y, j, v)=\operatorname{bit}^{f r}(y, j, v), \\
& \mathrm{bit}_{+\left(d+1 / 2^{k}\right)}^{f_{r}}(y, j, v)=\exists u\left(( u = \ell - k ) \wedge \left(\left((j \leq u) \wedge \mathrm{bit}_{+d}^{f_{r}}(y, j, v)\right) \vee\right.\right. \\
& \left((v=0) \wedge \mathrm{bit}_{+d}^{f_{r}}(y, j, 0) \wedge \exists j^{\prime}\left(\left(u<j^{\prime}<j\right) \wedge \mathrm{bit}_{+d}^{f_{r}}\left(y, j^{\prime}, 0\right)\right)\right) \vee \\
& \left((v=0) \wedge \mathrm{bit}_{+d}^{f r}(y, j, 1) \wedge \forall j^{\prime}\left(\left(u<j^{\prime}<j\right) \rightarrow \operatorname{bit}_{+d}^{f r}\left(y, j^{\prime}, 1\right)\right)\right) \vee \\
& \left((v=1) \wedge \operatorname{bit}_{+d}^{f r}(y, j, 1) \wedge \exists j^{\prime}\left(\left(u<j^{\prime}<j\right) \wedge \mathrm{bit}_{+d}^{f r}\left(y, j^{\prime}, 0\right)\right)\right) \vee \\
& \left.\left.\left((v=1) \wedge \mathrm{bit}_{+d}^{f r}(y, j, 0) \wedge \forall j^{\prime}\left(\left(u<j^{\prime}<j\right) \rightarrow \mathrm{bit}_{+d}^{f r}\left(y, j^{\prime}, 1\right)\right)\right)\right)\right), \\
& \operatorname{bit}_{+0 / 2^{k}}^{i n}(y, j, v)=\operatorname{bit}^{i n}(y, j, v), \\
& \mathrm{bit}_{+\left(d+1 / 2^{k}\right)}^{i n}(y, j, v)=\exists u((u=\ell-k) \wedge( \\
& \left(( v = 0 ) \wedge \operatorname { b i t } _ { + d } ^ { i n } ( y , j , 0 ) \wedge \exists j ^ { \prime } \left(\left(\left(j^{\prime}<j\right) \wedge \operatorname{bit}_{+d}^{i n}\left(y, j^{\prime}, 0\right)\right) \vee\right.\right. \\
& \left.\left.\left(\left(u<j^{\prime}<j\right) \wedge \mathrm{bit}_{+d}^{f r}\left(y, j^{\prime}, 0\right)\right)\right)\right) \vee \\
& \left(( v = 0 ) \wedge \operatorname { b i t } _ { + d } ^ { i n } ( y , j , 1 ) \wedge \forall j ^ { \prime } \left(\left(\left(j^{\prime}<j\right) \rightarrow \operatorname{bit}_{+d}^{i n}\left(y, j^{\prime}, 1\right)\right) \wedge\right.\right. \\
& \left.\left.\left(u<j^{\prime}<j\right) \rightarrow \operatorname{bit}_{+d}^{f r}\left(y, j^{\prime}, 1\right)\right)\right) \vee \\
& \left(( v = 1 ) \wedge \operatorname { b i t } _ { + d } ^ { i n } ( y , j , 0 ) \wedge \exists j ^ { \prime } \left(\left(\left(j^{\prime}<j\right) \wedge \operatorname{bit}_{+d}^{i n}\left(y, j^{\prime}, 0\right)\right) \vee\right.\right. \\
& \left.\left.\left(\left(u<j^{\prime}<j\right) \wedge \operatorname{bit}_{+d}^{f r}\left(y, j^{\prime}, 0\right)\right)\right)\right) \vee \\
& \left(( v = 1 ) \wedge \operatorname { b i t } _ { + d } ^ { i n } ( y , j , 1 ) \wedge \forall j ^ { \prime } \left(\left(\left(j^{\prime}<j\right) \rightarrow \operatorname{bit}_{+d}^{i n}\left(y, j^{\prime}, 1\right)\right) \wedge\right.\right. \\
& \left.\left.\left.\left.\left(\left(u<j^{\prime}<j\right) \rightarrow \operatorname{bit}_{+d}^{f r}\left(y, j^{\prime}, 1\right)\right)\right)\right)\right)\right) .
\end{aligned}
$$

Here, $u=\ell-k$ can be easily defined using $<$ and $k$.

\section{Proofs of Lemmas 18 and 19}

Lemma. If T satisfies TOA, then a projection of T satisfying TOA can be computed in time $O\left(|T|_{o}^{2} \times\right.$ $\left.|T|_{t}\right)$. 
Proof. We first partition $T$ into a set of purely temporal tables $T_{c_{1}, \ldots, c_{m}}$ and compute the set of all individual tuples $\left(c_{1}^{\prime}, \ldots, c_{n}^{\prime}\right)$ that will appear in the projection $T^{\prime}$. Let $\left(c_{1}^{\prime}, \ldots, c_{n}^{\prime}\right)$ be one such tuple, and consider the tables $T_{c_{1}^{1}, \ldots, c_{m}^{1}}, \ldots, T_{c_{1}^{k}, \ldots, c_{m}^{k}}$ such that the projection of each $\left(c_{1}^{i}, \ldots, c_{m}^{i}\right)$ is precisely $\left(c_{1}^{\prime}, \ldots, c_{n}^{\prime}\right)$. Clearly, we have at most $|T|_{o}$ such tables. It is well-known that, for a pair of ordered tables $S$ and $S^{\prime}$, we can construct an ordered table that contains all the tuples $S \cup S^{\prime}$ in time $|S|+\left|S^{\prime}\right|$. We use this algorithm $k$ times to obtain an ordered table containing all the tuples of $T_{c_{1}^{1}, \ldots, c_{m}^{1}} \cup \cdots \cup T_{c_{1}^{k}, \ldots, c_{m}^{k}}$ in time $O\left(k|T|_{o}\right)$. We then write the tuples of the form $\left(c_{1}^{\prime}, \ldots, c_{n}^{\prime},\left\langle, t_{1}, t_{2},\right\rangle\right)$, where $\left(\left\langle, t_{1}, t_{2},\right\rangle\right)$ is a tuple from the united table, into the output table. It can be readily checked that the complete output table can be produced in the required time.

Lemma. For any pair of tables $T$ and $T^{\prime}$ satisfying TOA, their union table also satisfying TOA can be computed in time $O\left(\left(|T|_{o}^{2}+\left|T^{\prime}\right|_{o}^{2}\right) \times\left(|T|_{t}+\left|T^{\prime}\right|_{t}\right)\right)$.

Proof. We first partition $T$ and $T^{\prime}$ into sets of purely temporal tables $T_{c_{1}, \ldots, c_{m}}$ and, respectively, $T_{c_{1}, \ldots, c_{m}}^{\prime}$. While doing this partition, we make sure that the tables $T_{c_{1}, \ldots, c_{m}}$ are stored sequentially with respect to some order on the tuples $\left(c_{1}, \ldots, c_{m}\right)$ (it can be done in time $\left.|T|_{o}^{2} \times|T|_{t}\right)$. We do the same for the tables $T_{c_{1}, \ldots, c_{m}}^{\prime}$. It remains to go through all the tuples $\left\langle, t_{1}, t_{2},\right\rangle$ and $\left\lceil, t_{1}^{\prime}, t_{2}^{\prime},\right\rceil$ in all the tables $T_{c_{1}, \ldots, c_{m}}$ and $T_{c_{1}, \ldots, c_{m}}^{\prime}$ to produce the union table by an algorithm similar to the one applied to the tables $S$ and $S^{\prime}$ in the proof of Lemma 18 .

\section{Experimental Results}

\begin{tabular}{|c|c|c|c|c|c|c|c|c|c|c|}
\hline queries $\quad$ \# of months & 32 & 64 & 96 & 128 & 159 & 191 & 223 & 255 & 287 & 320 \\
\hline ActivePowerTrip & 324 & 648 & 970 & 1294 & 1618 & 1940 & 2264 & 2588 & 2912 & 3236 \\
\hline NormalStop & 648 & 1296 & 1940 & 2588 & 3236 & 3880 & 4528 & 5176 & 5824 & 6472 \\
\hline NormalStart & 162 & 324 & 485 & 647 & 809 & 970 & 1132 & 1294 & 1456 & 1618 \\
\hline NormalRestart & 0 & 0 & 0 & 0 & 0 & 0 & 0 & 0 & 0 & 0 \\
\hline
\end{tabular}

Table 10: Number of the results returned from the Siemens queries.

\begin{tabular}{|l|rrrrrrrrrr|}
\hline \multicolumn{1}{|c|}{ \# of years } & 1 & 2 & 3 & 4 & 5 & 6 & 7 & 8 & 9 & 10 \\
\hline queries & & & & & & & & & & \\
\hline ShoweryPatternCounty & 530 & 1221 & 1802 & 2647 & 3609 & 4349 & 5204 & 5912 & 6639 & 7655 \\
HurricaneAffectedState & 2 & 4 & 5 & 5 & 5 & 8 & 9 & 801 & 1523 & 1533 \\
HeatAffectedCounty & 0 & 5 & 7 & 14 & 21 & 33 & 39 & 51 & 57 & 59 \\
CyclonePatternState & 914 & 1574 & 1617 & 1851 & 1936 & 2139 & 2246 & 2307 & 2333 & 2359 \\
\hline
\end{tabular}

Table 11: Number of the results returned from the NY weather stations from 2005 to 2014. 


\begin{tabular}{|l|rrrrrrrrrr|}
\hline \multicolumn{1}{|c|}{ \# of states } & 1 & 2 & 4 & 6 & 8 & 10 & 12 & 14 & 17 & 19 \\
\hline queries & & & & & & & & & & \\
\hline ShoweryPatternCounty & 3769 & 4481 & 4928 & 10349 & 12709 & 13681 & 14470 & 14933 & 16381 & 16883 \\
HurricaneAffectedState & 2 & 784 & 789 & 789 & 790 & 790 & 798 & 811 & 813 & 813 \\
HeatAffectedCounty & 53 & 65 & 81 & 84 & 88 & 98 & 100 & 117 & 142 & 224 \\
CyclonePatternState & 9109 & 9179 & 9593 & 17577 & 30203 & 38421 & 40769 & 43662 & 54199 & 56303 \\
\hline
\end{tabular}

Table 12: Number of the results returned from the Weather data for 1-19 states in 2012.

\begin{tabular}{|c|c|c|c|c|c|c|c|c|c|c|c|}
\hline & \# of months & 32 & 64 & 96 & 128 & 159 & 191 & 223 & 255 & 287 & 320 \\
\hline & \multirow[t]{2}{*}{ \# of rows } & 12,935, & 25,871, & 38,726, & 51,662 , & 64,597, & 77,453, & 90,389, & 103,324 , & 116,260 , & 129,195, \\
\hline & & 538 & 076 & 765 & 303 & 841 & 530 & 068 & 606 & 144 & 682 \\
\hline CSV & size $(\mathrm{GB})$ & 0.57 & 1.2 & 1.7 & 2.3 & 2.9 & 3.4 & 4.0 & 4.5 & 5.1 & 5.7 \\
\hline \multirow{2}{*}{ PostgreSQL } & raw size (GB) & 0.7 & 1.4 & 2.2 & 2.9 & 3.7 & 4.4 & 5.2 & 5.9 & 6.7 & 7.4 \\
\hline & total size (GB) & 1.0 & 2.0 & 3.0 & 4.0 & 5.0 & 6.0 & 7.0 & 8.0 & 9.0 & 10.0 \\
\hline Parquet & size $(\mathrm{GB})$ & 0.1 & 0.2 & 0.3 & 0.4 & 0.5 & 0.6 & 0.7 & 0.8 & 0.9 & 1.0 \\
\hline
\end{tabular}

Table 13: Siemens data for one turbine.

\begin{tabular}{|c|c|c|c|c|c|c|c|c|c|c|c|}
\hline & $\begin{array}{l}\text { \# of years } \\
\# \text { of stations }\end{array}$ & $\begin{array}{c}1 \\
229\end{array}$ & $\begin{array}{c}2 \\
306\end{array}$ & $\begin{array}{c}3 \\
370\end{array}$ & $\begin{array}{c}4 \\
441\end{array}$ & $\begin{array}{c}5 \\
484\end{array}$ & $\begin{array}{c}6 \\
542\end{array}$ & $\begin{array}{c}7 \\
595\end{array}$ & $\begin{array}{c}8 \\
643\end{array}$ & $\begin{array}{c}9 \\
807\end{array}$ & $\begin{array}{c}10 \\
874\end{array}$ \\
\hline & \# of rows & $\begin{array}{c}3,969, \\
455\end{array}$ & $\begin{array}{c}10,959, \\
978\end{array}$ & $\begin{array}{c}18,614, \\
686\end{array}$ & $\begin{array}{c}26,622, \\
218\end{array}$ & $\begin{array}{c}35,862, \\
560\end{array}$ & $\begin{array}{c}49,115, \\
307\end{array}$ & $\begin{array}{c}63,469 \\
733\end{array}$ & $\begin{array}{c}79,032, \\
846\end{array}$ & $\begin{array}{c}99,221, \\
419\end{array}$ & $\begin{array}{c}124,001, \\
260\end{array}$ \\
\hline CSV & size $(\mathrm{GB})$ & 0.2 & 0.6 & 1.1 & 1.6 & 2.1 & 2.9 & 3.8 & 4.8 & 5.9 & 7.4 \\
\hline PostgreSQL & $\begin{array}{l}\text { raw size }(\mathrm{GB}) \\
\text { total size }(\mathrm{GB})\end{array}$ & $\begin{array}{l}0.3 \\
0.4\end{array}$ & $\begin{array}{l}0.8 \\
1.1\end{array}$ & $\begin{array}{l}1.4 \\
2.0\end{array}$ & $\begin{array}{l}2.0 \\
2.9\end{array}$ & $\begin{array}{l}2.7 \\
3.9\end{array}$ & $\begin{array}{l}3.7 \\
5.4\end{array}$ & $\begin{array}{l}4.9 \\
7.1\end{array}$ & $\begin{array}{l}6.1 \\
8.9\end{array}$ & $\begin{array}{c}7.7 \\
11.0\end{array}$ & $\begin{array}{l}11.0 \\
14.0\end{array}$ \\
\hline Parquet & size $(\mathrm{GB})$ & 0.03 & 0.08 & 0.15 & 0.2 & 0.3 & 0.4 & 0.5 & 0.6 & 0.8 & 0.9 \\
\hline
\end{tabular}

Table 14: NY weather stations from 2005 to 2014.

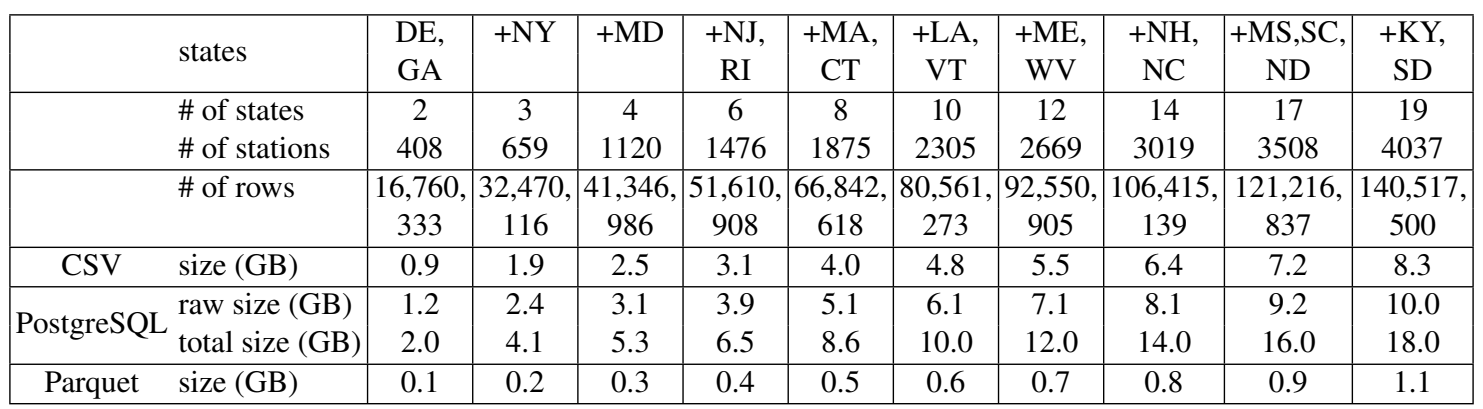

Table 15: Weather data for 1-19 states in 2012.

Here, CSV is the size of the data in CSV format; PostgreSQL (raw size) is the size of the data itself stored in PostgreSQL reported by the pg_relation_size function; PostgreSQL (total size) is the size of the total data (including the index) stored in PostgreSQL reported by the pg_total_relation_size function; and Parquet is the size of the data in the Apache Parquet format, used by Apache Spark. 


\section{References}

Abiteboul, S., Hull, R., \& Vianu, V. (1995). Foundations of Databases. Addison-Wesley.

Alur, R., Feder, T., \& Henzinger, T. A. (1996). The benefits of relaxing punctuality. J. ACM, 43(1), $116-146$.

Alur, R., \& Henzinger, T. A. (1993). Real-time logics: Complexity and expressiveness. Inf. Comput., 104(1), 35-77.

Armbrust, M., Xin, R. S., Lian, C., Huai, Y., Liu, D., Bradley, J. K., Meng, X., Kaftan, T., Franklin, M. J., Ghodsi, A., \& Zaharia, M. (2015). Spark SQL: relational data processing in spark. In Sellis, T. K., Davidson, S. B., \& Ives, Z. G. (Eds.), Proceedings of the 2015 ACM SIGMOD International Conference on Management of Data, Melbourne, Victoria, Australia, May 31 June 4, 2015, pp. 1383-1394. ACM.

Arora, S., \& Barak, B. (2009). Computational Complexity: A Modern Approach (1st edition). Cambridge University Press, New York, USA.

Artale, A., Kontchakov, R., Wolter, F., \& Zakharyaschev, M. (2013). Temporal description logic for ontology-based data access. In Proc. of the 23rd Int. Joint Conf. on Artificial Intelligence, IJCAI 2013. IJCAI/AAAI.

Artale, A., Kontchakov, R., Kovtunova, A., Ryzhikov, V., Wolter, F., \& Zakharyaschev, M. (2015). First-order rewritability of temporal ontology-mediated queries. In Proc. of the 24th Int. Joint Conf. on Artificial Intelligence, IJCAI 2015, pp. 2706-2712. IJCAI/AAAI.

Artale, A., Kontchakov, R., Kovtunova, A., Ryzhikov, V., Wolter, F., \& Zakharyaschev, M. (2017). Ontology-mediated query answering over temporal data: A survey (invited talk). In TIME, Vol. 90 of LIPIcs, pp. 1:1-1:37. Schloss Dagstuhl - Leibniz-Zentrum fuer Informatik.

Artale, A., Kontchakov, R., Ryzhikov, V., \& Zakharyaschev, M. (2013). The complexity of clausal fragments of LTL. In Logic for Programming, Artificial Intelligence, and Reasoning - 19th International Conference, LPAR-19, Stellenbosch, South Africa, December 14-19, 2013. Proceedings, pp. 35-52.

Artale, A., Kontchakov, R., Ryzhikov, V., \& Zakharyaschev, M. (2014). A cookbook for temporal conceptual data modelling with description logics. ACM Trans. Comput. Log., 15(3), 25:125:50.

Baader, F., Borgwardt, S., \& Lippmann, M. (2013). Temporalizing ontology-based data access. In Proc. of the 24th Int. Conf. on Automated Deduction, CADE-24, Vol. 7898 of LNCS, pp. 330-344. Springer.

Baudinet, M., Chomicki, J., \& Wolper, P. (1993). Temporal deductive databases. In Temporal Databases, pp. 294-320.

Beck, H., Dao-Tran, M., Eiter, T., \& Fink, M. (2015). LARS: A logic-based framework for analyzing reasoning over streams. In Bonet, B., \& Koenig, S. (Eds.), Proceedings of the Twenty-Ninth AAAI Conference on Artificial Intelligence, January 25-30, 2015, Austin, Texas, USA., pp. 1431-1438. AAAI Press.

Bienvenu, M., Kikot, S., Kontchakov, R., Podolskii, V. V., \& Zakharyaschev, M. (2018). Ontologymediated queries: Combined complexity and succinctness of rewritings via circuit complexity. J. ACM. In print. 
Borgwardt, S., Lippmann, M., \& Thost, V. (2013). Temporal query answering in the description logic DL-Lite. In Proc. of the 9th Int. Symposium on Frontiers of Combining Systems, FroCoS'13, Vol. 8152 of LNCS, pp. 165-180. Springer.

Brandt, S., Kalaycı, E. G., Kontchakov, R., Ryzhikov, V., Xiao, G., \& Zakharyaschev, M. (2017). Ontology-based data access with a horn fragment of metric temporal logic. In Singh, S. P., \& Markovitch, S. (Eds.), Proceedings of the Thirty-First AAAI Conference on Artificial Intelligence, February 4-9, 2017, San Francisco, California, USA., pp. 1070-1076. AAAI Press.

Bresolin, D., Kurucz, A., Muñoz-Velasco, E., Ryzhikov, V., Sciavicco, G., \& Zakharyaschev, M. (2017). Horn fragments of the halpern-shoham interval temporal logic. ACM Trans. Comput. Log., 18(3), 22:1-22:39.

Calvanese, D., Cogrel, B., Komla-Ebri, S., Kontchakov, R., Lanti, D., Rezk, M., Rodriguez-Muro, M., \& Xiao, G. (2017). Ontop: Answering SPARQL queries over relational databases. Semantic Web, 8(3), 471-487.

Chomicki, J., \& Toman, D. (1998). Temporal logic in information systems. In Logics for Databases and Information Systems, pp. 31-70. Kluwer.

Furia, C. A., \& Spoletini, P. (2008). Tomorrow and all our yesterdays: MTL satisfiability over the integers. In Fitzgerald, J. S., Haxthausen, A. E., \& Yenigün, H. (Eds.), Theoretical Aspects of Computing - ICTAC 2008, 5th International Colloquium, Istanbul, Turkey, September 1-3, 2008. Proceedings, Vol. 5160 of Lecture Notes in Computer Science, pp. 126-140. Springer.

Gabbay, D., Kurucz, A., Wolter, F., \& Zakharyaschev, M. (2003). Many-Dimensional Modal Logics: Theory and Applications, Vol. 148 of Studies in Logic and the Foundations of Mathematics. Elsevier.

Gottlob, G., Kikot, S., Kontchakov, R., Podolskii, V. V., Schwentick, T., \& Zakharyaschev, M. (2014). The price of query rewriting in ontology-based data access. Artif. Intell., 213, 42-59.

Gutiérrez-Basulto, V., Jung, J., \& Kontchakov, R. (2016a). Temporalized EL ontologies for accessing temporal data: Complexity of atomic queries. In Proceedings of the 25th International Joint Conference on Artificial Intelligence (IJCAI-16). AAAI Press.

Gutiérrez-Basulto, V., Jung, J. C., \& Ozaki, A. (2016b). On metric temporal description logics. In ECAI, Vol. 285 of Frontiers in Artificial Intelligence and Applications, pp. 837-845. IOS Press.

Gutiérrez-Basulto, V., \& Klarman, S. (2012). Towards a unifying approach to representing and querying temporal data in description logics. In Proc. of the 6th Int. Conf. on Web Reasoning and Rule Systems, RR 2012, Vol. 7497 of LNCS, pp. 90-105. Springer.

Kharlamov, E., Brandt, S., Jiménez-Ruiz, E., Kotidis, Y., Lamparter, S., Mailis, T., Neuenstadt, C., Özçep, Ö. L., Pinkel, C., Svingos, C., Zheleznyakov, D., Horrocks, I., Ioannidis, Y. E., \& Möller, R. (2016). Ontology-based integration of streaming and static relational data with optique. In Proc. of the 2016 Int. Conf. on Management of Data, SIGMOD Conference 2016, pp. 2109-2112.

Kharlamov, E., Mailis, T., Mehdi, G., Neuenstadt, C., Özçep, Ö. L., Roshchin, M., Solomakhina, N., Soylu, A., Svingos, C., Brandt, S., Giese, M., Ioannidis, Y. E., Lamparter, S., Möller, R., Kotidis, Y., \& Waaler, A. (2017). Semantic access to streaming and static data at siemens. $J$. Web Sem., 44, 54-74. 
Klarman, S., \& Meyer, T. (2014). Querying temporal databases via OWL 2 QL. In Proc. of the 8th Int. Conf. on Web Reasoning and Rule Systems, RR 2014, Vol. 8741 of LNCS, pp. 92-107. Springer.

Kontchakov, R., Rezk, M., Rodriguez-Muro, M., Xiao, G., \& Zakharyaschev, M. (2014). Answering SPARQL queries over databases under OWL 2 QL entailment regime. In Proc. of the 13th Int. Semantic Web Conf. (ISWC 2014), Part I, Vol. 8796 of LNCS, pp. 552-567. Springer.

Kontchakov, R., Pandolfo, L., Pulina, L., Ryzhikov, V., \& Zakharyaschev, M. (2016). Temporal and spatial OBDA with many-dimensional halpern-shoham logic. In IJCAI, pp. 1160-1166. IJCAI/AAAI Press.

Koymans, R. (1990). Specifying real-time properties with metric temporal logic. Real-Time Systems, 2(4), 255-299.

Ladner, R. E. (1977). The computational complexity of provability in systems of modal propositional logic. SIAM Journal of Computing.

Lutz, C., Wolter, F., \& Zakharyaschev, M. (2008). Temporal description logics: A survey. In Proc. of the 15th Int. Symposium on Temporal Representation and Reasoning (TIME 2008), pp. 3-14.

Madnani, K., Krishna, S. N., \& Pandya, P. K. (2013). On the decidability and complexity of some fragments of Metric Temporal Logic. CoRR, abs/1305.6137.

Ouaknine, J., \& Worrell, J. (2005). On the decidability of metric temporal logic. In Proceedings of the 20th Annual IEEE Symposium on Logic in Computer Science, LICS '05, pp. 188-197, Washington, DC, USA. IEEE Computer Society.

Ouaknine, J., \& Worrell, J. (2008). Some recent results in metric temporal logic. In Formal Modeling and Analysis of Timed Systems, 6th International Conference, FORMATS 2008, Saint Malo, France, September 15-17, 2008. Proceedings, pp. 1-13.

Özçep, Ö., Möller, R., Neuenstadt, C., Zheleznyakov, D., \& Kharlamov, E. (2013). A semantics for temporal and stream-based query answering in an OBDA context. Tech. rep., Deliverable D5.1, FP7-318338, EU.

Özçep, Ö. L., \& Möller, R. (2014). Ontology based data access on temporal and streaming data. In the 10th Int. Summer School on Reasoning Web, RW 2014, Vol. 8714 of LNCS, pp. 279-312. Springer.

Poggi, A., Lembo, D., Calvanese, D., De Giacomo, G., Lenzerini, M., \& Rosati, R. (2008). Linking data to ontologies. J. on Data Semantics, X, 133-173.

PostgreSQL (2018). Documentation 9.6.10. https : / /www • postgresql . org/docs/9.6/ static/parallel-safety.html.

Raheb, K. E., Mailis, T., Ryzhikov, V., Papapetrou, N., \& Ioannidis, Y. E. (2017). Balonse: Temporal aspects of dance movement and its ontological representation. In ESWC (2), Vol. 10250 of Lecture Notes in Computer Science, pp. 49-64.

Rodriguez-Muro, M., Kontchakov, R., \& Zakharyaschev, M. (2013). Ontology-based data access: Ontop of databases. In The Semantic Web - ISWC 2013 - 12th International Semantic Web Conference, Sydney, NSW, Australia, October 21-25, 2013, Proceedings, Part I, pp. 558-573.

Sistla, A., \& Clarke, E. (1985). The complexity of propositional linear temporal logics. J. ACM, 32, 733-749. 
Soylu, A., Giese, M., Jiménez-Ruiz, E., Vega-Gorgojo, G., \& Horrocks, I. (2016). Experiencing OptiqueVQS: a multi-paradigm and ontology-based visual query system for end users. Universal Access in the Information Society, 15(1), 129-152.

Tobies, S. (2001). Pspace reasoning for graded modal logics. Journal of Logic and Computation, 11(1), 85-106.

Ullman, J. D. (1988). Principles of Database and Knowledge-Base Systems, Volume I. Computer Science Press.

Vardi, M. (1982). The complexity of relational query languages (extended abstract). In Proc. of the 14th ACM SIGACT Symp. on Theory of Computing (STOC'82), pp. 137-146.

Xiao, G., Calvanese, D., Kontchakov, R., Lembo, D., Poggi, A., Rosati, R., \& Zakharyaschev, M. (2018). Ontology-based data access: A survey. In Proc. of the 28th Int. Joint Conf. on Artificial Intelligence (IJCAI). IJCAI/AAAI.

Zaharia, M., Xin, R. S., Wendell, P., Das, T., Armbrust, M., Dave, A., Meng, X., Rosen, J., Venkataraman, S., Franklin, M. J., Ghodsi, A., Gonzalez, J., Shenker, S., \& Stoica, I. (2016). Apache spark: a unified engine for big data processing. Commun. ACM, 59(11), 56-65.

Zhou, X., Wang, F., \& Zaniolo, C. (2006). Efficient temporal coalescing query support in relational database systems. In Proc. of the 17th Int. Conf. on Database and Expert Systems Applications, DEXA 2006, Vol. 4080 of LNCS, pp. 676-686. Springer. 